MCTP-11-01

UTTG-01-11

\title{
Heating up the Baryonic Branch with U-duality: a unified picture of conifold black holes
}

\author{
Elena Cáceres*1 ${ }^{*}$ Carlos Núñez ${ }^{\dagger 2}$ and Leopoldo A. Pando Zayas **3 \\ * Facultad de Ciencias \\ Universidad de Colima \\ Bernal Diaz del Castillo 340, Colima, México. \\ and \\ Theory Group, Department of Physics, \\ University of Texas at Austin, Austin, TX 78727, USA. \\ $\dagger$ Department of Physics \\ University of Swansea, Singleton Park \\ Swansea SA2 8PP \\ United Kingdom. \\ ** Michigan Center for Theoretical Physics \\ Randall Laboratory of Physics, the University of Michigan \\ Ann Arbor, MI 48109-1040. USA
}

\begin{abstract}
We study different aspects of a U-duality recently presented by Maldacena and Martelli and apply it to non-extremal backgrounds. In particular, starting from new non-extremal wrapped D5 branes we generate new non-extremal generalizations of the Baryonic Branch of the Klebanov-Strassler solution. We also elaborate on different conceptual aspects of these U-dualities, like its action on (extremal and non-extremal) Dp branes, dual models for YangMills-like theories, generic asymptotics and decoupling limit of the generated solutions.
\end{abstract}

\footnotetext{
${ }^{1}$ elenac@zippy.ph.utexas.edu

${ }^{2}$ c.nunez@swansea.ac.uk

${ }^{3}$ lpandoz@umich.edu
} 


\section{Contents}

1 Introduction $\quad 2$

1.1 General Idea of this Paper . . . . . . . . . . . . . . . . . . . 3

2 A warm-up example: Rotation of Dp-branes 4

2.1 "Rotation" in p directions . . . . . . . . . . . . . . . . . 4

2.1.1 Example: rotation of a dual to a Yang-Mills-like theory . . . . . . . . 7

2.2 "Rotation" in p-q directions . . . . . . . . . . . . . . . . . . 8

3 Black Holes in $\mathcal{N}=1$ SUSY Solutions $\quad 9$

3.1 A new 'seed' solution . . . . . . . . . . . . . . . . . . . . . 11

3.2 Asymptotics . . . . . . . . . . . . . . . . . . . . . 13

3.2.1 UV expansion . . . . . . . . . . . . . . . . . . . 13

3.2 .2 Near Horizon Asymptotics . . . . . . . . . . . . . . . . . . . . . . . . . 13

3.3 Numerics ............................. . . . 14

3.3.1 Comments about the numerical method . . . . . . . . . . . 14

3.4 The rotated solution . . . . . . . . . . . . . . . . . 15

3.5 Comments on thermodynamics . . . . . . . . . . . . . . . . . . 16

4 About decoupling limits $\quad 19$

4.1 Choice of Parameters for $\mathcal{N}=1$ Black Holes . . . . . . . . . . . . . . . . . . 20

5 Conclusions and Future Directions $\quad 21$

A Appendix: U-duality in the case of flat Dp-branes 23

B Appendix: Black Dp branes $\quad 24$

C Appendix: Some details on the U-duality 25

D Appendix: Another solution generating algorithm 27

E Appendix: U-duality for the wrapped D5 branes Black Hole 29

F Appendix: The equations of motion $\quad 31$ 


\section{Introduction}

The Maldacena conjecture [1] and some of its refinements [2] are the guiding principle behind much of the progress in the interface String Theory-Quantum Field theory in the last twelve years. The influence of this approach extends to toy models with different number of supersymmetries (SUSY's), systems at finite temperature and/or finite density and lower dimensional systems. The applications to physically relevant systems do not seem to be exhausted and on the contrary, increase with time. In this sense, finding new (trustable) solutions to the equations of motion of String Theory (even in the point particle and classical approximation) has become an industry with various applications in Physics and Mathematics. Solution generating techniques have certainly played a role. Some examples worth mentioning are the combination of T-dualities and shifts of coordinates that generated solutions dual to minimally SUSY superconformal field theories (beta deformations) or duals to non-relativistic field theories $[3]^{1}$.

In this paper we will focus our attention on a particular solution generating technique that was presented in [6]. The procedure suggested by these authors starts by taking a solution in type IIB string theory proposed to be dual to (a suitably UV-completed version of) $\mathcal{N}=1$ Super Yang-Mills in four dimensions. The solution has the topology $R^{1,3} \times M_{6}$ where $M_{6}$ preserves four supercharges. The algorithm to generate the new solutions goes as follows: first apply a set of three T-dualities in the $R^{3}$ directions, which leaves us with a IIA configuration; lift this configuration to M-theory and boost-with rapidity $\beta$-in the eleventh direction; then reduce to IIA and T-dualize back in $R^{3}$. This generates a solution that roughly speaking is the dual description to the baryonic branch [7] of the Klebanov-Strassler field theory [8]. For all the technical details of this procedure the reader can refer to [6] or to our Appendix E.

The 'seed solution' (as we will refer to the initial solution on which the generating algorithm is applied) was discussed in the papers [9] (Section 8) and [10] (Section 4.3). In a particular limit, this becomes the exact solution discussed in [11].

We will also refer to the solution generating algorithm, that is a U-duality, as 'rotation' in a sense explained below and in previous papers. In the following we will emphasize some aspects of the generating technique presented in [6] that we find particularly interesting and have not been explicitly discussed in the existing bibliography:

- In the case of [6], the rotation generates D3 brane charge. This is the reason why the $S O(1,3)$ isometry of the background is untouched, in spite of doing different operations in time (boost) and in the $R^{3}$ directions (T-dualities). As a by-product of the generation of D3 branes the dilaton is invariant under this set of operations.

\footnotetext{
${ }^{1}$ In other areas of Physics, solution generating techniques play a major role. For example, many of the interesting solutions in higher dimensional gravity were constructed using solution generating algorithms. See for example [4] for original papers and [5] for a nice summary with an important application.
} 
- It can be seen that this U-duality (sequence of T-dualities, lifts and boosts) described above is equivalent to a particular case of 'rotation' in the $S U(3)$ structure of the manifold $M_{6}$ which is characterized by a complex 3 -form $\Omega_{3}$ and a 2 -form $J_{2}$ - see the papers [12] and [13]. This is another way of understanding the presence of the $S O(1,3)$ isometry: from this perspective, all the rotation 'occurs' in the internal $M_{6}$.

- The rotation generates a solution that contains two free parameters, the value of the dilaton at infinity (already present in the seed solution) and the boost parameter $\beta$. In order for the final background to be dual to the Klebanov-Strassler QFT decoupled from gravity, we must take the limit $\beta \rightarrow \infty$ so that the generated warp factor vanishes asymptotically. In this sense, the seed solution describes a field theory coupled to gravity. Only in a particular limit-see the discussion in [6], [13], we approach the near brane solution of [11].

- This rotation or U-duality, is a very curious operation from the viewpoint of the dual field theory. Indeed, it generates (aside from the bifundamental fields) new global symmetries, like the baryonic $U(1)$.

- In the same vein: the connection between the two field theories from a geometric viewpoint (Field theory at strong coupling!) is just the mentioned U-duality starting from the $D 5$-brane side. From a (weakly interacting) field theoretic viewpoint the operation is very subtle [6]. Indeed, it involves a second order fluctuation expansion (mass spectrum) in the classical and weakly coupled $\mathrm{F}$ and $\mathrm{D}$ term equations on the quiver side [15], that is matched with a classical and weakly coupled expansion of the twisted compactification of the theory in the wrapped D5 brane mass spectrum [16].

- After the first set of T-dualities described above, we have a IIA background with D2 brane charges. When lifted to M-theory we have M2 branes, that after boosted generate M2 charge and an M-wave. When reduced, this generates a D0, D2 and NS $H_{3}$ field. The generation of the $H_{3}$ in the presence of $\mathrm{RR}$ three-form implies the need to generate $F_{5}$. See Appendix E for technical details.

- We emphasized that the U-duality ( chain of T-dualities, lifts and boost) described above is a particular case of the rotation of $J_{2}, \Omega_{3}$ discussed in [12], [13], [14]. On the other hand, the rotation of $J_{2}, \Omega_{3}$ relies on the background being supersymmetric, while the U-duality can be also applied to Non-SUSY backgrounds. This will play an important role in the rest of this paper.

\subsection{General Idea of this Paper}

In this paper we will try to gain a different perspective on this solution generating technique. We will not focus on the rotation of $J, \Omega$ but rely on the U-duality description (in spite of the 
latter being a particular case of the former). Our interests will be two-fold. On one hand, we will try to get a better handle on the generating algorithm by changing it (suggesting other related algorithms) and applying it to various cases. On the other hand, we will apply it to backgrounds that do not preserve SUSY. We will study the effect of the rotation on black hole solutions, generating new non-extremal solutions with horizons and other curious features that will be discussed. We will also start the study of the properties of those newly generated solutions.

This paper is organized as follows: In sections 2 and 2.1 as a warm-up exercise we discuss a chain of dualities and boost acting on non-extremal Dp branes. In section 2.1.1 we will apply this to rotate a background dual to a version of Yang-Mills. Then, in section 2.2 we will study a variation of this U-duality that will clarify various aspects of the coming material. In Section 3 we will U-dualize a new solution describing the non-extremal deformation of a stack of $N_{c}$ D5 branes wrapping a two-cycle inside the resolved conifold. We will elaborate upon various aspects of this particular rotation in section 3.5. Finally in Section 4 we comment on decoupling aspects of the rotation. Various appendices complement our presentation. They have been written with plenty of detail hoping that colleagues working on these topics will find them useful. We close with a summary, conclusions and a list of possible future projects.

\section{A warm-up example: Rotation of Dp-branes}

In this section, we will start with a simple example of the 'rotation' procedure. That is, a sequence of T-dualities, bringing the background to a type IIA solution of the supergravity equations of motion, followed by a lift to eleven dimensions, where we will boost the configuration. We will then reduce to IIA and T-dualize back, to what we will call the 'rotated background'. As a toy example, in this section we will rotate flat $D p$ branes; first in $p$ directions and then in $p-q$ directions. We will do this in detail to appreciate the differences this introduces in the generated solution. In Appendix D, we will propose and analyze another possible U-duality to generate new solutions.

\section{1 "Rotation" in $\mathrm{p}$ directions}

Consider backgrounds of IIA/IIB of the form,

$$
\begin{aligned}
& d s^{2}=H(\rho)^{-1 / 2}\left[-h(\rho) d t^{2}+d \vec{x}_{p}^{2}\right]+H(\rho)^{1 / 2}\left[\frac{d \rho^{2}}{h(\rho)}+\rho^{2} d \Omega_{8-p}\right] \\
& e^{2 \phi[\text { initial }]}=e^{2 \phi(\infty)} H^{\frac{3-p}{2}} \\
& * F_{p+2}=\mathcal{Q} V_{o l} \Omega_{8-p}, \\
& F_{p+2}=-\partial_{\rho} A(\rho) d t \wedge d x_{1} \wedge \ldots \wedge d x_{p} \wedge d \rho
\end{aligned}
$$


with,

$$
H(\rho)=1+\left(\frac{L_{p}}{\rho}\right)^{7-p}, h(\rho)=1-\left(\frac{R_{T}}{\rho}\right)^{7-p}, \quad A(\rho)=\frac{\alpha}{g_{s} H(\rho)},
$$

where $\alpha=\frac{\tilde{Q}}{L_{p}^{7-p}}$ and $\mathcal{Q}=(7-p) \tilde{Q}$ is related to the charge of the solution (see Appendix B for details). If we choose $R_{T}=0$, the configuration above is typically singular (except for $p=3$ ) and preserves 16 SUSYs. Recall that for even (odd) values of $p$, we are dealing with a solution of Type IIA(B) supergravity.

Now, we will 'rotate' this background, by first T-dualizing in the $p$ directions, this will bring us to a IIA solution, we will lift the solution to eleven-dimensional supergravity and perform a boost of rapidity $\beta$ in the eleventh-direction. We will then reduce to IIA and T-dualize back in the $\vec{x}_{p}$ coordinates to obtain what we call the 'rotated background'. Our final rotated background is given $\mathrm{by}^{2}$,

$$
\begin{aligned}
& d s^{2}=\frac{H(\rho)^{-1 / 2}}{R(\rho)}\left[-h(\rho) d t^{2}+d \vec{x}_{p}^{2}\right]+R(\rho) H(\rho)^{1 / 2}\left[\frac{d \rho^{2}}{h(\rho)}+\rho^{2} d \Omega_{8-p}\right] \\
& e^{2 \phi[\text { final }]}=e^{2 \phi[\text { initial }]} R(\rho)^{3-p}=e^{2 \phi(\infty)}\left(H(\rho) R(\rho)^{2}\right)^{\frac{3-p}{2}} \\
& F_{p+2}=-\partial_{\rho} a(\rho) d t \wedge d x_{1} \wedge \ldots \wedge d x_{p} \wedge d \rho
\end{aligned}
$$

where we have defined,

$$
\begin{aligned}
& R^{2}=(A(\rho) \sinh \beta+\cosh \beta)^{2}-\frac{h(\rho) \sinh ^{2} \beta}{g_{s}^{2} H(\rho)^{2}} \\
& a=\frac{1}{R^{2}}\left[A(\rho) \cosh 2 \beta+\left(A(\rho)^{2}+1\right) \cosh \beta \sinh \beta-\frac{h(\rho)}{2 g_{s}^{2} H(\rho)^{2}} \sinh (2 \beta)\right]
\end{aligned}
$$

We have explicitly checked that the rotated background is a solution of the equations of motion. Notice that eq. (2.6) can be written as

$$
R^{2}=\cosh ^{2} \beta+A(\rho) \sinh 2 \beta+\left(\frac{R_{T}}{L_{p}}\right)^{7-p} \frac{\sinh ^{2} \beta}{H(\rho) g_{s}^{2}}
$$

which makes clear that $R$ is strictly positive. In a similar way, eq. (2.7) can be written as

$$
a=\frac{1}{H R^{2}}\left[\frac{1}{g_{s}} \alpha \cosh (2 \beta)+\left(\frac{R_{T}}{L_{p}}\right)^{7-p} \frac{\sinh \beta \cosh \beta}{g_{s}^{2}}+H(\rho) \sinh \beta \cosh \beta\right] \text {. }
$$

The metric (2.3) has the structure of a warped space,

$$
d s^{2}=\mathcal{H}(\rho)^{-1 / 2}\left[-h(\rho) d t^{2}+d \vec{x}_{p}^{2}\right]+\mathcal{H}(\rho)^{1 / 2}\left[\frac{d \rho^{2}}{h(\rho)}+\rho^{2} d \Omega_{8-p}\right],
$$

\footnotetext{
${ }^{2}$ In Appendix A, we will present the intermediate steps of this calculation for the extremal case $h=1$.
} 
where the new warp factor is

$$
\mathcal{H}(\rho)=H(\rho) R(\rho)^{2}=H(\rho) \cosh ^{2} \beta+\frac{\alpha}{g_{s}} \sinh 2 \beta+\left(\frac{R_{T}}{L_{p}}\right)^{7-p} \frac{\sinh ^{2} \beta}{g_{s}^{2}}
$$

Note that $\mathcal{H}(\rho)$ is a harmonic function of the transverse space. Hence, after the rotation in $p$ directions we are left with a $D p$ brane solution. Indeed, in terms of the new warp factor, the dilaton and gauge potential are

$$
\begin{aligned}
& e^{2 \phi[\text { final }]}=e^{2 \phi(\infty)} \mathcal{H}(\rho)^{\frac{3-p}{2}} \\
& \partial_{\rho} a(\rho)=\left[\frac{\alpha}{g_{s}}+\left(\frac{R_{T}}{L_{p}}\right)^{7-p} \frac{\tanh \beta}{g_{s}^{2}}\right] \partial_{\rho}\left(\frac{1}{\mathcal{H}(\rho)}\right)=\tilde{\alpha} \partial_{\rho}\left(\frac{1}{\mathcal{H}(\rho)}\right),
\end{aligned}
$$

which together with (2.10) define a $D p$ brane background with a $\beta$ dependent $\mathrm{RR}$ charge. At infinity the warp factor asymptotes to a constant,

$$
\mathcal{H}(\rho) \sim \cosh ^{2} \beta+\frac{\alpha}{g_{s}} \sinh 2 \beta+\left(\frac{R_{T}}{L_{p}}\right)^{7-p} \frac{\sinh ^{2} \beta}{g_{s}^{2}},
$$

and the space is asymptotically flat, as expected. However, it is interesting to note that even if we start with the Dp branes after the decoupling limit is taken, that is $H=\frac{L_{p}^{7-p}}{\rho^{7-p}}$, we will have in the UV that,

$$
\mathcal{H}(\rho) \sim \frac{L_{p}^{7-p}}{\rho^{7-p}} \cosh ^{2} \beta+\frac{\alpha}{g_{s}} \sinh 2 \beta+\left(\frac{R_{T}}{L_{p}}\right)^{7-p} \frac{\sinh ^{2} \beta}{g_{s}^{2}}
$$

So, again, the warp factor asymptotes to a constant. In other words, this rotation is taking the configuration out of the decoupling limit or coupling the field theory modes to gravity.

As is well known, before the rotation, the charge of the Dp brane solution is quantized. After the rotation the charge is,

$$
\begin{aligned}
\frac{1}{\left(2 \pi l_{s}\right)^{7-p}} \int_{S^{8-p}} * F_{p+2} & =\tilde{\alpha} \rho^{8-p} \mathcal{H}^{\prime}(\rho) V o l_{S^{8-p}} \\
& =\mathrm{Q} \cosh ^{2} \beta+R_{T}{ }^{7-p} \frac{c_{p}}{2 g_{s}} \sinh 2 \beta
\end{aligned}
$$

where $c_{p}=\frac{(7-p) V o l_{S} 8-p}{\left(2 \pi l_{s}\right)^{7-p} g_{s}}$. Note that, generically, the charge is not quantized. This is not unusual since the supergravity dualities involved in the rotation procedure are a symmetry of the supergravity equations and not of the full string theory.

We will now move to study a more interesting example from the viewpoint of gauge-strings duality. We will apply the rotation procedure to the dual of a non-SUSY Yang-Mills-like theory, first presented in [17] and further studied in [18], [19]. 


\subsubsection{Example: rotation of a dual to a Yang-Mills-like theory}

The original background, consists of the decoupling limit of a stack of $N_{c}$ D4 branes wrapping a circle with SUSY breaking boundary conditions. It was discussed with details in various publication, see for example [17], [18], [19]. We summarize it here (in string frame $)^{3}$

$$
\begin{aligned}
& d s^{2}=H(\rho)^{-1 / 2}\left[-h(\rho) d t^{2}+d \vec{x}_{123}^{2}+f(\rho) d x_{4}^{2}\right]+H(\rho)^{1 / 2}\left[\frac{d \rho^{2}}{f(\rho) h(\rho)}+\rho^{2} d \Omega_{4}\right], \\
& e^{2 \phi[\text { initial }]}=g_{s}^{2} H^{-\frac{1}{2}} \\
& F_{6}=-\partial_{\rho} A(\rho) d t \wedge d x_{1} \wedge \ldots \wedge d x_{4} \wedge d \rho \quad F_{4}=* F_{6}
\end{aligned}
$$

where $A(\rho)=\frac{1}{g_{s} H(\rho)}$ and $V o l_{4}$ is the volume of the unit four- sphere $\Omega_{4}$.. The functions (in the low/zero temperature phase) are given by

$$
H=\left(\frac{L}{\rho}\right)^{3}, \quad f=1-\left(\frac{R_{k k}}{\rho}\right)^{3}, \quad h=1,
$$

where $L^{3}=N_{c} \pi g_{s} l_{s}^{3}$ and the coefficient $R_{k k}$ is a free parameter. In the high temperature phase (with the coordinate $x_{4}$ compactified) we have,

$$
H=\left(\frac{L}{\rho}\right)^{3}, \quad h=1-\left(\frac{R_{T}}{\rho}\right)^{3}, \quad f=1
$$

where $R_{T}$ is a free parameter related to the temperature of the system,

$$
T=\frac{3}{4 \pi}\left(\frac{L^{3}}{R_{T}}\right)^{-1 / 2}
$$

Proceeding as described in the previous section we rotate the background by applying the Uduality already discussed: four T-dualities in the worldvolume coordinates, uplift to M-theory, boost, reduce to IIA and T dualize back. The rotated background is,

$$
\begin{aligned}
& d s^{2}=\mathcal{H}(\rho)^{-1 / 2}\left[-h(\rho) d t^{2}+d \vec{x}_{123}^{2}+f(\rho) d x_{4}^{2}\right]+\mathcal{H}(\rho)^{1 / 2}\left[\frac{d \rho^{2}}{f(\rho) h(\rho)}+\rho^{2} d \Omega_{4}\right] \\
& e^{2 \phi[\text { final }]}=g_{s}^{2} \mathcal{H}^{-\frac{1}{2}} \\
& F_{6}=a^{\prime}(\rho) d t \wedge d x_{1} \wedge \ldots \wedge d x_{4} \wedge d \rho
\end{aligned}
$$

where the functions $\mathcal{H}(\rho), a(\rho)$ now read

$$
\begin{aligned}
& \mathcal{H}(\rho)=H(\rho) \cosh ^{2} \beta+\frac{1}{g_{s}} \sinh 2 \beta+\left(\frac{R_{T}}{L}\right)^{3} \frac{\sinh ^{2} \beta}{g_{s}^{2}} \\
& a(\rho)=\frac{1}{R(\rho)^{2}}\left[A(\rho) \cosh 2 \beta+\left(A(\rho)^{2}+1\right) \cosh \beta \sinh \beta-\frac{f(\rho) h(\rho) \sinh (2 \beta)}{2 g_{s}^{2} H(\rho)^{2}}\right]
\end{aligned}
$$

\footnotetext{
${ }^{3}$ In [18] the authors performed a rescaling of the RR potential: $C_{p+1} \rightarrow \frac{\kappa_{0}^{2} \mu_{6-p}}{\pi} C_{p+1}$ and as a result the $\mathrm{RR}$ charge in their paper is measured in units of $2 \pi$. We do not perform such rescaling here.
} 
and $R(\rho)^{2}=\frac{\mathcal{H}(\rho)}{H(\rho)}$. As before, one can check explicitly that the background above is a solution of the eqs. of motion, but for this we must set either $R_{T}=0$ for the low/zero temperature phase or set $R_{k k}=0$, compactifying the $x_{4}$ direction, in the high temperature phase.

As explained in the previous section, in spite of having started with a decoupled background, after the rotation we have an asymptotically flat space, indicating that the rotation has coupled the field theory modes to the gravitational ones.

\section{2 "Rotation" in p-q directions}

We will study now a peculiar situation, where we have Dp branes and we separate a $q$ manifold from the $p+1$ dimensional world-volume (we focus in the case $q=2$ here, the reason for this will be explained in Appendix C). The internal manifold need not be specified. We will impose on our U-duality that:

- after the initial T-dualities, we are in IIA background, so that this is upliftable to Mtheory

- that the boost generates a NS $H_{3}$ field, or what is equivalent, that the IIA configuration after the initial T-dualities contains an electric $F_{4}$.

We will assume (though this need not be the case) that the internal q-manifold is a torus. In that case, the solution reads (in string frame as usual) ${ }^{4}$,

$$
\begin{aligned}
& d s^{2}=H^{-1 / 2}\left[-d t^{2}+d x_{p-q}^{2}+d \sigma_{q}^{2}\right]+H^{1 / 2}\left[d \rho^{2}+\rho^{2} d \Omega_{8-p}^{2}\right] \\
& F_{p+2}=\partial_{\rho} \hat{A} d t \wedge d x_{1} \wedge \ldots \wedge d x_{p-q} \wedge d \sigma_{q} \wedge d \rho, \\
& e^{2 \phi[\text { initial }]}=e^{2 \phi(\infty)} H^{\frac{3-p}{2}} .
\end{aligned}
$$

We will proceed as follows: first we will do $\mathrm{T}$ dualities in the $x_{p-q}$ directions, this will leave us in a IIA configuration, we will lift then to M-theory and boost. We reduce to IIA and T dualize back, all the details are written in Appendix C. We end up this duality-chain with a final configuration

$$
\begin{aligned}
& d s_{I I, s t}^{2}=g_{t t} d t^{2}+\frac{d \vec{x}_{p-2}^{2}}{H^{1 / 3} B^{1 / 2}}+B^{1 / 2}\left(H^{-2 / 3} d \vec{\sigma}_{2}^{2}+H^{1 / 3}\left[d \rho^{2}+\rho^{2} d \Omega_{8-p}^{2}\right]\right), \\
& F_{p+2}=\partial_{\rho} \hat{A}\left(\cosh \beta+a_{t} \sinh \beta\right) \wedge d \rho \wedge d \sigma_{1} \wedge d \sigma_{2} \wedge d x_{1} \wedge \ldots \wedge d x_{p-2}, \\
& H_{3}=\sinh \beta \partial_{\rho} \hat{A} d \rho \wedge d \sigma_{1} \wedge d \sigma_{2}, \\
& F_{p}=\partial_{\rho}\left(a_{t}\right) d t \wedge d \rho \wedge d x_{1} \wedge \ldots \wedge \wedge d x_{p-2}, \\
& e^{2 \phi[\text { final }]}=B^{\frac{5-p}{2}} H^{\frac{2-p}{3}}
\end{aligned}
$$

\footnotetext{
${ }^{4}$ we are dealing here with the Lorentz invariant case. Working with a non-extremal factor is straightforward.
} 
where we have defined (See Appendix C for a detailed derivation),

$$
\begin{aligned}
& A=H^{-2 / 3}\left[H \sinh ^{2} \beta-\cosh ^{2} \beta\right], \quad B=H^{-2 / 3}\left[H \cosh ^{2} \beta-\sinh ^{2} \beta\right], \\
& C=2 H^{-2 / 3} \sinh \beta \cosh \beta(1-H), \quad g_{t t}=\frac{4 A B-C^{2}}{4 \sqrt{B}}, \\
& a_{t}=\frac{C}{2 B}=\frac{\sinh \beta \cosh \beta(1-H)}{H \cosh ^{2} \beta-\sinh ^{2} \beta},
\end{aligned}
$$

Various aspects are worth emphasizing in this final background. Notice that:

- the metric is dual to a field theory with $S O(1, p-2)$ isometry, since

$$
\left(4 A B-C^{2}\right) H^{1 / 3}=-4 \rightarrow g_{t t}=-g_{x_{i} x_{i}}
$$

- for $p=5$ the dilaton is invariant under the whole U-duality procedure, since

$$
e^{2 \phi[\text { initial }]}=H^{\frac{3-p}{2}}=e^{2 \phi[\text { final }]}=B^{\frac{5-p}{2}} H^{\frac{2-p}{3}}
$$

- for $p=5$ the procedure is generating charge of D3 brane (represented by the $F_{p=5}$ ) where the D3 branes are extended in the $p-q$ directions. This is the reason why the dilaton does not change as the D3 branes do not couple to it. The field $H_{3}$ is also generated.

In following sections, we will study a particular case of this U-duality, for a situation in which a set of D5 branes wrap a curved two-manifold (but we also have a black hole in the metric, breaking the $S O(1,3)$ isometry), the results are qualitatively the same. One should emphasize that there is yet another way of generating NS three-form fields, that is basically starting from NS five branes in IIA wrapping a three-cycle, see [20] for details.

Also, notice that we have imposed that after the first set of T-dualities, the background is solution of IIA Supergravity (the conditions for this to happen are discussed in Appendix C). Were this not the case, we present in Appendix D another possible solution generating technique with some applications.

Now, we will move to study an interesting application of the U-dualities discussed above.

\section{Black Holes in $\mathcal{N}=1$ SUSY Solutions}

As anticipated, in this section we will apply the chain of dualities, lift and boost proposed in [6], to generate a new solution starting from a non-extremal solution in Type IIB. The interest of the original ('seed') solution is that it was argued to be dual to a field theory with minimal SUSY in four dimensions. The field theory was studied (at weak coupling) in [16] and is basically $\mathcal{N}=1$ Super-Yang-Mills plus a sets of (massive) KK vector and chiral superfields 
that UV complete the dynamics. This system was well studied and various string duals are known (particular solutions describing the field theory at strong coupling, with VEV's and certain operators deforming the Lagrangian). Let us briefly describe the general form of the string dual. The 'seed' background describes the backreaction of a set of $N_{c}$ D5 branes wrapping a two-cycle inside the resolved conifold. It consists of a metric, dilaton $\phi(\rho)$ and RR three-form $F_{3}$ and, in string frame, it reads (the coordinates used are $[t, \vec{x}, \rho, \theta, \varphi, \tilde{\theta}, \tilde{\varphi}, \psi]$ ),

$$
\begin{aligned}
d s^{2}= & e^{\phi(\rho)}\left[d x_{1,3}^{2}+e^{2 k(\rho)} d \rho^{2}+e^{2 h(\rho)}\left(d \theta^{2}+\sin ^{2} \theta d \varphi^{2}\right)+\right. \\
+ & \left.\frac{e^{2 g(\rho)}}{4}\left(\left(\tilde{\omega}_{1}+a(\rho) d \theta\right)^{2}+\left(\tilde{\omega}_{2}-a(\rho) \sin \theta d \varphi\right)^{2}\right)+\frac{e^{2 k(\rho)}}{4}\left(\tilde{\omega}_{3}+\cos \theta d \varphi\right)^{2}\right], \\
F_{(3)}= & \frac{N_{c}}{4}\left[-\left(\tilde{\omega}_{1}+b(\rho) d \theta\right) \wedge\left(\tilde{\omega}_{2}-b(\rho) \sin \theta d \varphi\right) \wedge\left(\tilde{\omega}_{3}+\cos \theta d \varphi\right)+\right. \\
& \left.b^{\prime} d \rho \wedge\left(-d \theta \wedge \tilde{\omega}_{1}+\sin \theta d \varphi \wedge \tilde{\omega}_{2}\right)+\left(1-b(\rho)^{2}\right) \sin \theta d \theta \wedge d \varphi \wedge \tilde{\omega}_{3}\right] .
\end{aligned}
$$

where $\tilde{\omega}_{i}$ are the left-invariant forms of $S U(2)$

$$
\begin{aligned}
& \tilde{\omega}_{1}=\cos \psi d \tilde{\theta}+\sin \psi \sin \tilde{\theta} d \tilde{\varphi}, \quad \tilde{\omega}_{2}=-\sin \psi d \tilde{\theta}+\cos \psi \sin \tilde{\theta} d \tilde{\varphi}, \\
& \tilde{\omega}_{3}=d \psi+\cos \tilde{\theta} d \tilde{\varphi} .
\end{aligned}
$$

This supersymmetric system was carefully studied in a series of papers [9], [10]; where it was shown that there is a combination of background functions (basically a 'change of basis') that move from a set of coupled BPS equations to a decoupled one that one can solve-up to one function, that we will call $P(\rho)$. We will not insist much with this formalism here and refer the interested reader to the original work [9], $[10]^{5}$.

We will be more restrictive and for the purposes of this paper, we will study solutions where the functions $a(\rho)=b(\rho)=0$ in the background of eq.(3.28). This is just in order to make our numerics simpler and illustrate the points we want to make. If we wanted to construct a black hole solution showing explicitly the transition between R-symmetry breaking and its restoration, we should then work without this restriction. Since in all known solutions the asymptotics of the functions $a(\rho) \sim b(\rho) \sim e^{-2 \rho}$ our asymptotic results will be qualitatively correct, but near the black hole horizon there could be important differences.

We will then proceed as follows: first we will propose a background including a black hole with the restrictions mentioned above $(a(\rho)=b(\rho)=0)$. We will then pass it through the solution generating machine. This will produce a new background, now including also $F_{5}, H_{3}$, that we will write explicitly (we have checked that the equations of motion before and after the U-duality are the same). We will be explicit about the asymptotics of each of the functions

\footnotetext{
${ }^{5}$ All this formalism was also applied to the case in which one also adds fundamental matter to the dual QFT or sources to the background above. We refer the interested reader to the review [21].
} 
near the UV and near the horizon. This will produce for us a non-extremal generalization of the type IIB string background dual to the 'baryonic branch of KS-system' -certainly with the restriction that our background does not contain the information of the breaking of the R-symmetry from $Z_{2 N} \rightarrow Z_{2}$.

\subsection{A new 'seed' solution}

In this section we consider the background presented in equation (3.28) with the restrictions $a=b=0$ but including the non-extremality factors. In string frame we have,

$$
\begin{aligned}
& d s_{I I B, s}^{2}=e^{\phi}\left[-h(\rho) d t^{2}+d x_{1}^{2}+d x_{2}^{2}+d x_{3}^{2}\right]+d s_{6, s}^{2}, \\
& d s_{6}^{2}=e^{\phi}\left[\frac{e^{2 k}}{s(\rho)} d \rho^{2}+\frac{e^{2 k}}{4}\left(\tilde{\omega}_{3}+\cos \theta d \varphi\right)^{2}+e^{2 q}\left(d \theta^{2}+\sin ^{2} \theta d \varphi^{2}\right)+\frac{e^{2 g}}{4}\left(d \tilde{\theta}^{2}+\sin ^{2} \tilde{\theta} d \tilde{\varphi}^{2}\right)\right] \\
& F_{(3)}=\frac{N_{c}}{4}\left[-\tilde{\omega}_{1} \wedge \tilde{\omega}_{2}+\sin \theta d \theta \wedge d \varphi\right] \wedge\left(\tilde{\omega}_{3}+\cos \theta d \varphi\right)=\frac{\alpha^{\prime} N_{c}}{4} w_{3},
\end{aligned}
$$

where $h(\rho), s(\rho)$ are the non-extremality functions. In the following, we will set $s(\rho)=h(\rho)$ which is simply a choice of parametrization; this implies that (on a particular solution) other background functions $\{q(\rho), g(\rho), k(\rho), \phi(\rho)\}$ need not take the same values as in the SUSY background.

The above Ansatz might be familiar to some readers. It is worth highlighting a key difference with previous work. Since our goal is to 'rotate' this solution we look for solutions with stabilized dilaton. Namely, the typical asymptotic value of the dilaton inherited from the D5 (or NS5 brane) solution is linear [32], more precisely [23] [25],

$$
e^{\phi} \sim e^{\rho} \rho^{-1 / 4}
$$

We are looking for a dilaton that stabilizes at infinity, that is, which asymptotically behaves as:

$$
e^{\phi} \sim 1+\mathcal{O}\left(e^{-8 / 3 \rho}\right)
$$

Our solution is qualitatively characterized by two parameters: one describing the nonextremality of the solution and the other the speed at which the dilaton gets stabilized.

After applying the solution generating technique, we end up with a new Type IIB background containing $F_{5}, H_{3}$ aside from the fields originally present in eq.(3.30) (all details are 
written in the Appendix E),

$$
\begin{aligned}
& d s_{I I B, s t}^{2}=H^{-1 / 2}\left[-h d t^{2}+d x_{1}^{2}+d x_{2}^{2}+d x_{3}^{2}\right]+e^{2 \phi} H^{1 / 2}\left[e^{2 k} \frac{d \rho^{2}}{h(\rho)}+\frac{e^{2 k}}{4}\left(\tilde{\omega}_{3}+\cos \theta d \varphi\right)^{2}+\right. \\
& \left.+e^{2 q}\left(d \theta^{2}+\sin ^{2} \theta d \varphi^{2}\right)+\frac{e^{2 g}}{4}\left(d \tilde{\theta}^{2}+\sin ^{2} \tilde{\theta} d \tilde{\varphi}^{2}\right)\right] \\
& H^{1 / 2}=\cosh \beta \sqrt{e^{-2 \phi}-h(\rho) \tanh ^{2} \beta}, \quad \phi_{n e w}=\phi(\rho), \\
& F_{3}=\left(\frac{\alpha^{\prime} N_{c}}{4}\right) \cosh \beta w_{3}, \quad H_{3}=-\sqrt{h}\left(\frac{\alpha^{\prime} N_{c}}{4}\right) \sinh \beta e^{2 \phi} *_{6} w_{3} \\
& F_{5}=-\tanh \beta\left(1+*_{10}\right) \operatorname{vol}_{4} \wedge d\left(\frac{h}{H}\right) .
\end{aligned}
$$

In the following, we will focus in the limit $\beta \rightarrow \infty$, this is the field theory-limit where the warp factors vanish at infinity. We will then rescale

$$
\tilde{N}=N \cosh \beta, \quad x_{i} \rightarrow \sqrt{\cosh \beta} \sqrt{\tilde{N} \alpha^{\prime}} x .
$$

With all the above the $\beta \rightarrow \infty$ limits are finite and the solution is given by

$$
\begin{aligned}
& d s_{I I B, s t}^{2}=\tilde{N} \alpha^{\prime}\left[\tilde{H}^{-1 / 2}\left(-h d t^{2}+d x_{i} d x^{i}\right)+e^{2 \phi} \tilde{H}^{1 / 2} d s_{6}^{2}\right], \\
& F_{3}=\frac{\alpha^{\prime} \tilde{N}}{4} w_{3}, \quad H_{3}=-\sqrt{h} \frac{\alpha^{\prime} \tilde{N}}{4} e^{2 \phi} *_{6} w_{3} \\
& F_{5}=-\left(\alpha^{\prime} \tilde{N}\right)^{2}\left(1+*_{10}\right) \operatorname{vol}_{4} \wedge d\left(\frac{h}{\tilde{H}}\right)
\end{aligned}
$$

with

$$
\tilde{H}^{1 / 2}=\sqrt{e^{-2 \phi}-h(\rho)}
$$

It is worth making a few observations:

- We have two new classes of solutions. The equations of motion before and after the 'rotation' are the same, thus a solution of the system of equations (3.30) furnishes a solution of the form (3.33)-(3.35).

- The non-extremality factor have made its way into the RR five form and the warp factor in eq.(3.36), but the dilaton and the three forms are unchanged from the extremal case. Notice that the factor of $\sqrt{h}$ in the NS field $H_{3}$ is canceled by the self dual in six dimensions $*_{6} w_{3}$. See Appendix E for full details.

- The black hole in the seed solution will likely have negative specific heat but its dilaton is stabilized which is a crucial difference with solutions considered previously. We will discuss what is the behavior of the solution after the rotation.

In the following section we will explicitly describe the asymptotic behavior of the solution as well as its numerical presentation in the background given by eq.(3.30). 


\section{$3.2 \quad$ Asymptotics}

We will proceed to study the asymptotics of the equations of motion and solve them numerically to obtain the new non-extremal seed solution (3.30).

\subsubsection{UV expansion}

A large $\rho$ expansion of the functions $(k, q, g, h, \phi)$, that solve the equations of motion up to terms decaying faster than $e^{-4 \rho}$ is ${ }^{6}$

$$
\begin{aligned}
& h(\rho)=e^{-8 x(\rho)} \sim 1+C_{2} e^{-\frac{8}{3} \rho} \\
& e^{2 q(\rho)} \sim \frac{N_{c}}{4}(2 \rho-1)+\frac{c}{4} e^{\frac{4}{3} \rho}, \quad e^{2 g(\rho)} \sim \frac{N_{c}}{4}(1-2 \rho)+\frac{c}{4} e^{\frac{4}{3} \rho} \\
& e^{2 k(\rho)} \sim \frac{2 c}{3} e^{\frac{4}{3} \rho}-\frac{N_{c}^{2}}{6 c}\left(25-40 \rho+16 \rho^{2}\right) e^{-\frac{4}{3} \rho}, \quad e^{4 \phi-4 \phi_{0}} \sim 1+\frac{3 N_{c}^{2}}{4 c^{2}}(1-8 \rho) e^{-\frac{8}{3} \rho}
\end{aligned}
$$

There are two parameters in the expansion above. The parameter $C_{2}$ characterizes the nonextremal behavior. The parameter $c$ plays a quite important role. It is one of the integration constants of the BPS eqs. As an expansion in inverse powers of this constant it is possible to write a solution for the background in eq.(3.28)-see for example [10], [22]-such that when passed through this solution generating technique, it results in the Baryonic Branch dual solution of [7]. Physically (and in the background before the rotation), the constant $c$ is describing the coupling of the dynamics on the five-brane to gravity and ultimately to the full string theory, it takes the configuration out of its 'near brane' limit and can be thought of as indicating the insertion of an irrelevant operator in the QFT lagrangian.

\subsubsection{Near Horizon Asymptotics}

Near the horizon, $\rho=\rho_{h}$, we expand the equations of motion in power series up to order 7 . We have found,

$$
\begin{aligned}
e^{-8 x(\rho)} & =x_{1}\left(\rho-\rho_{h}\right)+x_{2}\left(\rho-\rho_{h}\right)^{2}+x_{3}\left(\rho-\rho_{h}\right)^{3}+\cdots+x_{8}\left(\rho-\rho_{h}\right)^{8} \\
e^{2 q(\rho)} & =q_{0}+q_{1}\left(\rho-\rho_{h}\right)+q_{2}\left(\rho-\rho_{h}\right)^{2}+\cdots+q_{7}\left(\rho-\rho_{h}\right)^{7} \\
e^{2 g(\rho)} & =g_{0}+h_{1}\left(\rho-\rho_{h}\right)+g_{2}\left(\rho-\rho_{h}\right)^{2}+\cdots+g_{7}\left(\rho-\rho_{h}\right)^{7} \\
e^{2 k(\rho)} & =k_{0}+k_{1}\left(\rho-\rho_{h}\right)+k_{2}\left(\rho-\rho_{h}\right)^{2}+\cdots+k_{7}\left(\rho-\rho_{h}\right)^{7} \\
e^{4 \Phi(\rho)} & =f_{0}+f_{1}\left(\rho-\rho_{h}\right)+f_{2}\left(\rho-\rho_{h}\right)^{2}+\cdots+f_{7}\left(\rho-\rho_{h}\right)^{7}
\end{aligned}
$$

Demanding that the expansions in eq.(3.38) satisfy the equations of motion of the system we determine $x_{2}, \ldots, h_{1} \ldots, g_{1} \ldots, k_{1} \ldots, f_{1} \ldots$ in terms of $x_{1}, h_{0}, g_{0}, k_{0}, f_{0}$ and thus, there are only 5

\footnotetext{
${ }^{6}$ Note that the UV expansion for $h(\rho)=e^{-8 x}$ could have started with a lower order term such as $e^{-2 \rho}$, see Section 4.1 for a discussion on the choice of parameters.
} 

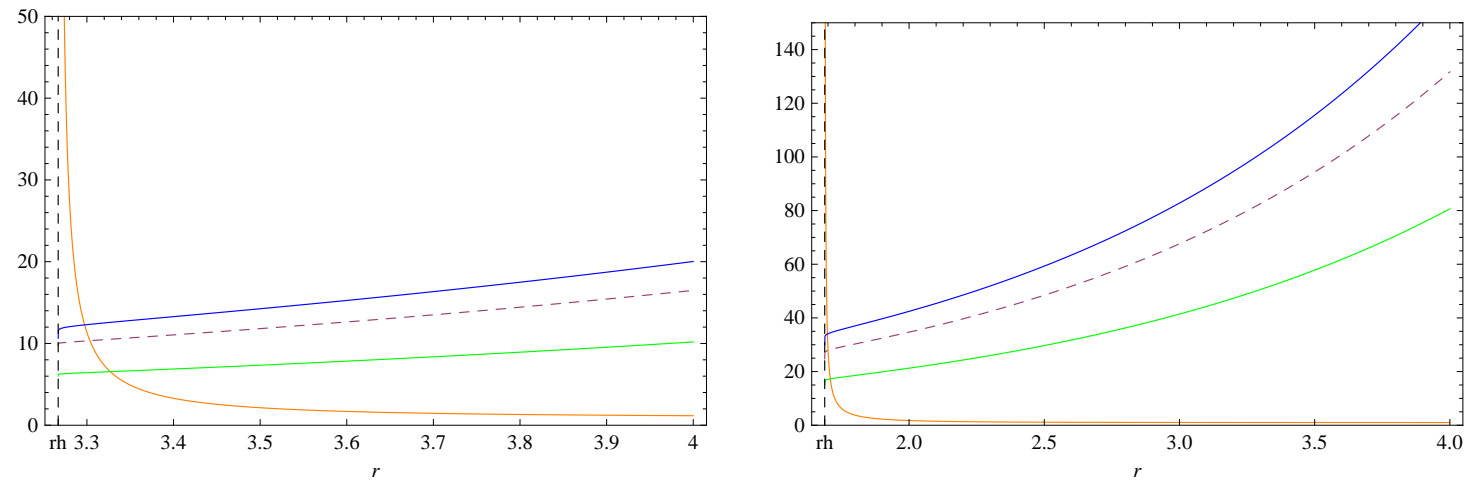

Figure 1: Solution before the rotation for Figure 2: Solution before the rotation for $c=1.97, C_{2}=-7000$. The blue, dashed, $c=125.62, C_{2}=-89$. The blue, dashed, green and orange lines represent $e^{q}, e^{k}, e^{g}$ and green and orange lines represent $e^{q}, e^{k}, e^{g}$ and $h^{-1}$ respectively. $\quad h^{-1}$ respectively.

independent parameters coming from the expansion at the horizon. Furthermore, $x_{1}$ is related to the non-extremality parameter $\alpha$-see Appendix F for the definitions,

$$
\alpha=-\frac{x_{1} \sqrt{f_{0}} g_{0} h_{0}}{8}
$$

\subsection{Numerics}

The strategy we will follow is to numerically integrate back from infinity the equations of motion in Appendix F, using as boundary conditions the expansion in the UV-eq.(3.37)-and require that at the horizon this numerical solution matches the expansion (3.38) and its first derivatives -up to seventh order. Doing this for a given set of values $\left(c, C_{2}\right)$, determines the free parameters $\left(x_{1}, h_{0}, g_{0}, k_{0}, f_{0}\right)$. Note that not for every pair of $\left(c, C_{2}\right)$ there is a black hole solution. For some values of $\left(c, C_{2}\right)$ there will not be a horizon and for others there might be naked singularities outside the horizon. The study of the this two-parameter family of solutions for the full $\left(c, C_{2}\right)$ parameter space is an interesting question that we will not address here. We concentrate on finding particular values of $\left(c, C_{2}\right)$ that will produce black hole solutions. Needless to say, finding a solution before the rotation guarantees that we will have a solution after the rotation. Examples of numerical solutions are shown in Figure 1 and 2 and the respective dilatons in Figures 3 and 4 .

\subsubsection{Comments about the numerical method}

As mentioned above we integrate back from infinity and match with the expansion at the horizon. In order to do so we define a "mismatch" function evaluated at a point $\rho_{0}$ close to 

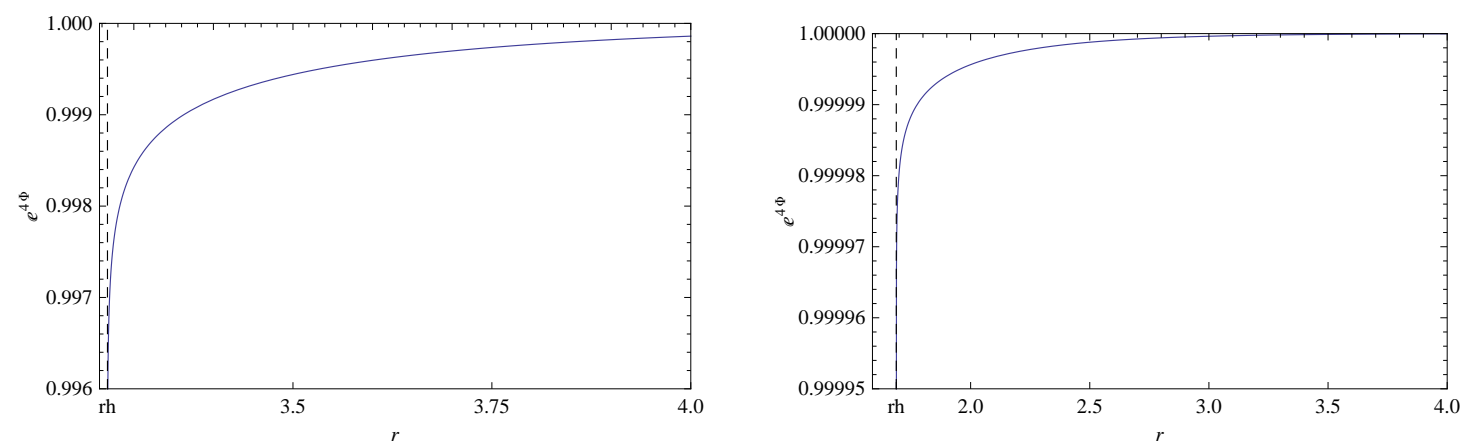

Figure 3: Dilaton, $e^{4 \Phi}$ for $c=1.97, C_{2}=$ Figure 4: Dilaton, $e^{4 \Phi}$ for $c=125.62, C_{2}=$ -7000 . -89 .

the horizon, $\rho_{0}=\rho_{h}+0.15$

$$
\begin{aligned}
m\left(\rho_{0}\right)= & {\left[g_{\text {sh }}\left(\rho_{0}\right)-g_{\text {num }}\left(\rho_{0}\right)\right]^{2}+\left[g_{\text {sh }}^{\prime}\left(\rho_{0}\right)-g_{\text {num }}^{\prime}\left(\rho_{0}\right)\right]^{2}+\left[h_{\text {sh }}\left(\rho_{0}\right)-h_{\text {num }}\left(\rho_{0}\right)\right]^{2}+} \\
& {\left[h_{\text {sh }}^{\prime}\left(\rho_{0}\right)-h_{\text {num }}^{\prime}\left(\rho_{0}\right)\right]^{2}+\left[k_{s h}\left(\rho_{0}\right)-k_{\text {num }}\left(\rho_{0}\right)\right]^{2}+\left[k_{\text {sh }}^{\prime}\left(\rho_{0}\right)-k_{\text {num }}^{\prime}\left(\rho_{0}\right)\right]^{2}+} \\
& {\left[\Phi_{\text {sh }}\left(\rho_{0}\right)-\Phi_{\text {num }}\left(\rho_{0}\right)\right]^{2}+\left[\Phi_{\text {sh }}^{\prime}\left(\rho_{0}\right)-\Phi_{\text {num }}^{\prime}\left(\rho_{0}\right)\right]^{2} . }
\end{aligned}
$$

The subscript num denotes the numerical solution obtained by integrating back from infinity and $s h$ denotes the series expansion (3.38) -evaluated at $\rho_{0}$. Recall that the expansion at the horizon depends on five free parameters, $\left(x_{1}, h_{0}, g_{0}, k_{0}, f_{0}\right)$. For a given set of $\left(c, C_{2}\right)$ we minimize the mismatch function to determine $\left(x_{1}, h_{0}, g_{0}, k_{0}, f_{0}\right)$. The parameters at infinity $\left(c, C_{2}\right)$ are adjusted so that $m\left(\rho_{0}\right)<5 \times 10^{-4}$. All the numerical procedures where done in Mathematica using WorkingPrecission 40. We also checked that the constraint $T+U=0$ (see appendix F) remains numerically negligible throughout the domain.

\subsection{The rotated solution}

Once we have obtained a numerical solution for the non-extremal background (3.30) we can easily generate the rotated solution as summarized in eq.(3.35) and outlined in the Appendix E. Before presenting the numerical results let us look at the large $\rho$ asymptotics. Using the UV expansions of the seed solution (3.37), one can obtain the black hole metric (3.35) asymptotics 
after the rotation,

$$
\begin{aligned}
-g_{t t} & \sim \frac{e^{4 / 3 \rho}}{A(\rho)}-\frac{e^{-4 / 3 \rho}}{512 c^{4} \sqrt{2} A(\rho)^{3}}\left[256 c^{4} C_{2}^{2}+27(1-8 \rho)^{2}+96 c^{2} C_{2}(-1+8 \rho)\right]+\cdots, \\
g_{x x} & \sim \frac{e^{4 / 3 \rho}}{A(\rho)}-\frac{e^{-4 / 3 \rho}}{512 c^{4} \sqrt{2} A(\rho)^{3}}\left[27(1-8 \rho)^{2}\right]+\cdots, \\
g_{\rho \rho} & \sim \frac{2 c}{3} A(\rho)+\cdots, \quad g_{\theta \theta} \sim \frac{c}{4} A(\rho)+\frac{e^{-4 / 3 \rho}}{4} A(\rho)(2 \rho-1)+\cdots, \\
g_{\psi \psi} & \sim \frac{c}{6} A(\rho)+\cdots, \quad g_{\tilde{\theta} \tilde{\theta}} \sim \frac{c}{4} A(\rho)-\frac{e^{-4 / 3 \rho}}{4} A(\rho)(2 \rho-1)+\cdots .
\end{aligned}
$$

Where we have defined the quantity

$$
c \sqrt{8} A(\rho) \equiv \sqrt{24 \rho-8 C_{2} c^{2}-3} .
$$

Using a variable $u=e^{2 \rho / 3}$ the metric can be written -to leading order- as,

$$
d s^{2}=\frac{u^{2}}{A(u)}\left(d x_{i} d x^{i}\right)+\frac{3 c A(u)}{2}\left(\frac{d u^{2}}{u^{2}}+d s_{T^{11}}^{2}\right)+\cdots \mathcal{O}\left(u^{-2}\right) .
$$

Thus, the non-extremal solution found after the rotation has the asymptotics of the KlebanovStrassler background [8]. We point out that the reason we get precisely Klebanov-Tseytlin type of asymptotics lies in our precise arrangements of coefficients in the seed solution. Namely we arranged for a seed solution with no linear dilaton and with a dilaton behavior that, in the field theory limit, eliminates the leading term in the harmonic function given in equation $(3.36)$.

With our careful choice of asymptotic conditions for the seed solution of D5 branes we have managed to construct black holes in asymptotically KT backgrounds. Our solution is different from those obtained in the traditional approach to black holes in asymptotically KT backgrounds (see, for example, [33]). One glaring difference is the form of the RR fluxes. In particular the presence of the non-extremality parameter in $F_{5}$ and in the warp factor $H(\rho)$ is completely novel.

In Figures 5-8 we present the numerical plots of the diffent metric elements after the rotation for two sets of parameters. In Figures 9 and 10 we see how the angular part of the solution approaches the $T^{1,1}$ metric for the two representative solutions.

\subsection{Comments on thermodynamics}

Let us clarify the expectations of the form of the background that arise from field theory considerations. In the previous section we obtained a solution largely characterized by two parameters $\left(c, C_{2}\right)$. After the rotation, the interpretation of $c$ has been spelled out explicitly 

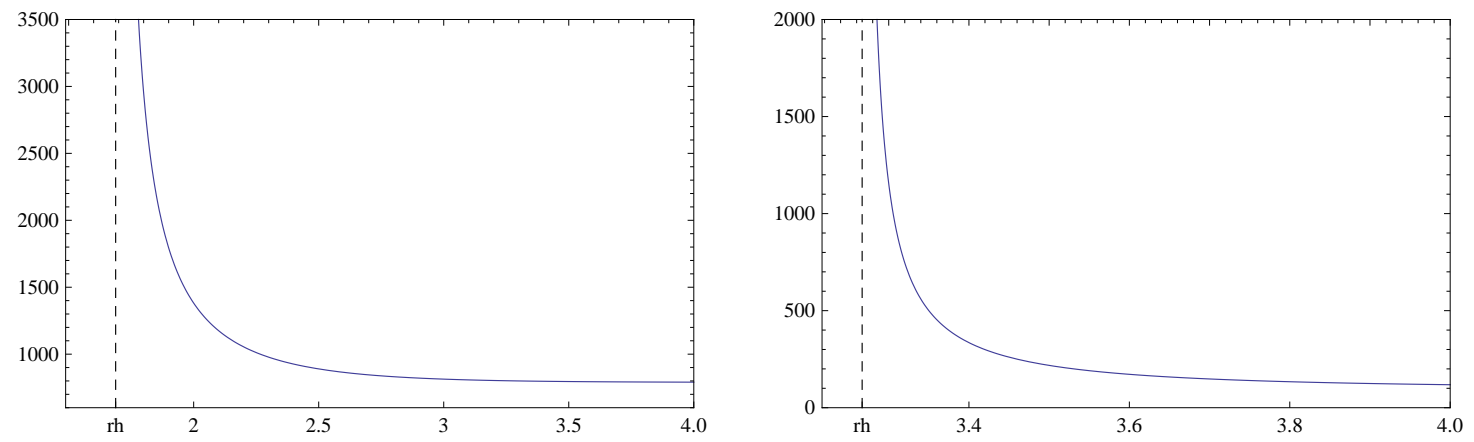

Figure 5: The $g_{r r}$ metric element for a so- Figure 6: The $g_{r r}$ metric element for a solulution after the rotation, $c=125.62, C 2=$ tion after the rotation, $c=1.97, C_{2}=-7000$. -89 .
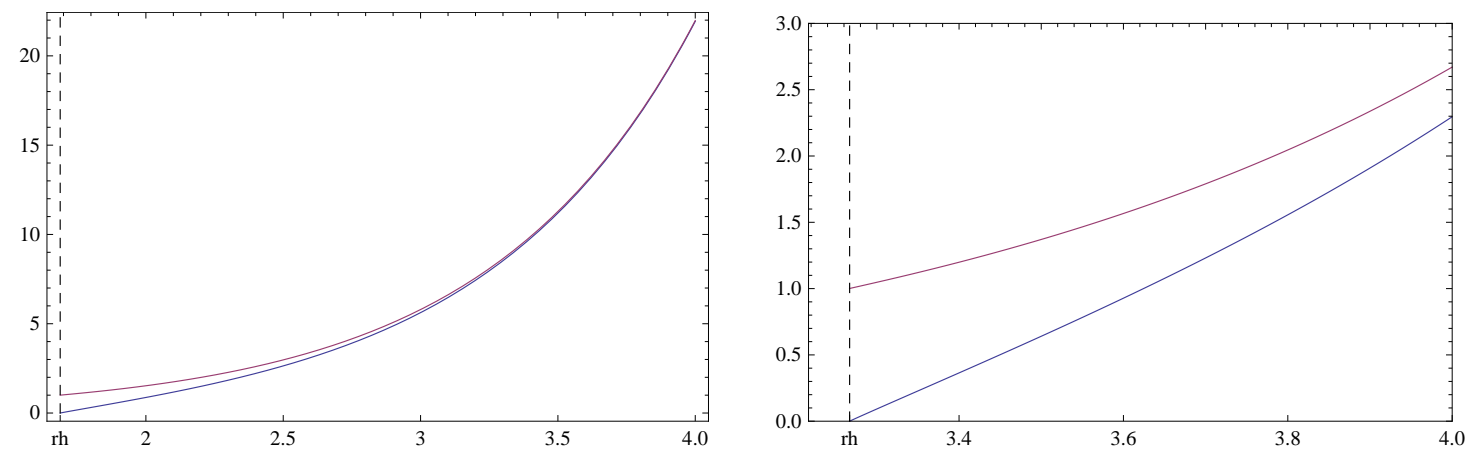

Figure 7: $g_{x x}$ and $g_{t t}$ for a solution after the Figure 8: $g_{x x}$ and $g_{t t}$ for a solution after the rotation, $c=125.62, C_{2}=-89$. rotation, $c=1.97, C_{2}=-7000$.


Figure 9: Angular part of the metric after Figure 10: Angular part of the metric after the rotation. We plot $g_{\theta \theta}, g_{\tilde{\theta} \tilde{\theta}}$ and $\frac{3}{2} g_{\psi \psi}$. So- the rotation. We plot $g_{\theta \theta}, g_{\tilde{\theta} \tilde{\theta}}$ and $\frac{3}{2} g_{\psi \psi}$. Solution with $c=125, C_{2}=-89$. lution with $c=1.97, C_{2}=-7000$. 
in [15], [6] and [13]. It is related to the vev of the baryon operator. The other parameter present in the solution is $C_{2}$, its interpretation coincides roughly with the temperature of the solution in the field theory.

We will present and exhaustive analysis of these classes of solutions elsewhere and will limit our attention here to a few main points. The main deterrent in presenting a final description of the thermodynamics is the need to explore a large parameter space of the solutions as well as the difficulty in matching data at asymptotic infinity with data near the horizon. The analysis is numerically costly but we emphasize that these are not difficulties of principle. Our goal in this work is to explicitly show, through numerical analysis, the existence of the conjectured solutions.

Let us show that the thermodynamic analysis can, in principle, be done explicitly for the solutions we have. For the temperature, we use the standard approach of looking at the Euclidean section and imposing the absence of conical singularities to determine the temperature of the supergravity backgrounds. Basically, for a metric with Euclidean section of the form

$$
d s^{2}=f d \tau^{2}+\frac{d \rho^{2}}{g}+\ldots,
$$

we find a temperature equal to

$$
T=\frac{1}{4 \pi} \sqrt{f^{\prime} g^{\prime}},
$$

where prime represents derivative with respect to the radial coordinate and the above expression is evaluated at the horizon. Interestingly, the temperature in both backgrounds, that is, before and after the rotation, is the same. In terms of the near horizon data we have

$$
T=\frac{1}{4 \pi} \frac{x_{1}}{\sqrt{k_{0}}} .
$$

It is worth noting that this is the temperature at the horizon. In the case of asymptotically flat backgrounds (before the rotation) we have the option of computing the temperature as seen by an observer at infinity. We refer the reader to [23] for a detailed discussion of this procedure in the D5 or NS5 background. The entropy is computed, as usual, as the area of the horizon. The most complicated quantity to compute is the free energy which is required to compute, for example, the specific heat. For the free energy we need to evaluate the action. In the case of asymptotically flat solutions for D5/NS5 branes we can essentially follow [23]. A very preliminary analysis seems to confirm our intuition about the specific heat of such solutions. Namely, evaluation of the action suggests that before the rotation the solutions obtained have negative specific heat. A precise evaluation of the action after the rotation is more subtle as naive evaluation yields divergent terms. It is likely that a more accurate approach requires some sort of holographic renormalization or substraction along the lines of [33]. Preliminary evaluation suggests a positive specific heat for the rotated solutions ${ }^{7}$.

\footnotetext{
${ }^{7}$ Dario Martelli suggested that along the lines of the first two papers in [3], some thermodynamical quantities should be invariant under T-dualities, shifts of coordinates and boosts. See also [34].
} 


\section{About decoupling limits}

It is worth discussing a bit about decoupling limits. Let us do this in the extremal case, the extension to the cases with non extremal factors follows from what we write here. The first point to emphasize is that when gravity modes are coupled to a generic D-brane configuration, the warp factors asymptote to a constant. An example of this is what happens to the flat Dp branes, with $H=1+\left(\frac{Q}{\rho}\right)^{7-p}$.

We will compare warp factors in the examples studied in the previous sections. It is enough to focus on the expressions of the metric component $g_{t t}$. In all the examples studied we found

$$
g_{t t}=\frac{4 A B-C^{2}}{4 \sqrt{B}}
$$

Indeed, in the case in which we rotate a flat Dp-brane, we have eq.(A.6) with $A, B, C$ given in eq.(A.5). In the case of the rotation in $p-q$ directions the results for $A, B, C$ are written in eq. (2.25) and in the case of the wrapped D5's are given in eq.(E.9). Again, for the point we wish to emphasize, we will focus on the extremal cases, when the rotation preserves $S O(1, p)$. So, in these cases we have ${ }^{8}$,

$$
\begin{aligned}
& g_{t t,(p-q)}=-\frac{1}{\cosh \beta \sqrt{H-\tanh ^{2} \beta}}, g_{t t, p}=-\frac{\sqrt{H}}{\sqrt{H^{2}(\hat{A} \tanh \beta-1)^{2}-\tanh ^{2} \beta}}, \\
& g_{t t, D 5}=-\frac{1}{\cosh \beta \sqrt{e^{-2 \phi}-\tanh ^{2} \beta}}
\end{aligned}
$$

The case of the rotation in $p$ directions shows that the induced charge of D0 brane (after the first set of T-dualities) as shown by eq.(A.2), enters in the new warp factor via the one-form potential $A_{1}=\hat{A} d t$. This makes the decoupling of the final configuration more unclear and typically, if we start with 'decoupled' Dp branes (that is $H \sim \rho^{p-7}$ ) we will generate a nondecoupled configuration, with an asymptotically constant warp factor as discussed around eq.(2.13). Of course, we can try to take the decoupling limit in the final configuration.

On the other hand the cases of the wrapped D5's and the rotation in $p-q$ directions (that share the fact that a D0 charge is induced by the boost only) are such that we must start with a non-decoupled configuration in order to avoid a generated background with a singularity or end of the space in the UV (zero warp factor at some finite value of the radial coordinate). Indeed, if $H=1+\left(\frac{Q}{\rho}\right)^{7-p}$ we choose a particular value of the boost parameter $\beta \rightarrow \infty$, together with a particular choice of the value of $e^{\phi(\infty)}=1$ in the D5's case) to obtain a background decoupled from gravity ${ }^{9}$.

\footnotetext{
${ }^{8}$ Gregory Giecold correctly pointed out that in the non-extremal cases the non-extremality factor $h(\rho)$ enters as expected, for example $g_{t t, D 5}=-\frac{h}{\cosh \beta \sqrt{e^{-2 \phi}-h \tanh ^{2} \beta}}$. We thank him for this and various other correct comments.

${ }^{9}$ One of the interesting observations in [6], is that in the case of the D5's the solution described in the
} 


\subsection{Choice of Parameters for $\mathcal{N}=1$ Black Holes}

Let us discuss in more detail the choice of parameters in the solution. This is a particularly sensitive question from the numerical and from the physical points of view. We note that $a$ priori there are ten parameters in our solution as corresponds to a system of five second order ordinary differential equations. The constraint lowers this number by one. At this point the simplest route for us is to notice that we are looking for a particular solution that asymptotes to the extremal supersymmetric one found in [10],[13] (see, in particular, appendix B there). In that context most of our constants get fixed, as we require the same UV behavior; we use the expressions in that solution to fix six constants. Of the three constants left, one gets fixed by the asymptotic behavior of the non-extremality function, that is, we choose

$$
h(\rho) \sim 1-C_{2} e^{-\frac{8}{3} \rho} .
$$

The leading coefficient guarantees that we will have, after rotation, a solution with KT asymptotics. The key observation to be made is that, in principle, there is a mode allowed that we have explicitly set to zero. Namely, another solution to the equations of motion in the asymptotic regime could be

$$
h(\rho) \sim 1+C_{1} e^{-2 \rho}-C_{2} e^{-\frac{8}{3} \rho}+\ldots .
$$

The above expression should be thought of schematically as the solution to a second order differential equation for $h(\rho)$ which is defined asymptotically by two parameters. This $C_{1}$ is one of the three constants that we fix and therefore we remain with two constants that we called $\left(c, C_{2}\right)$. The parameter $c$ has the same meaning discussed in [10],[13] and $C_{2}$ is related to the temperature. Thus, the condition that the solution after the rotation has KT asymptotics plus a condition on the non-extremality function determine the values of the integration constants.

It is worth noting that selecting the right constants in the D3/D5 picture is more cumbersome as there are more parameters in the generic D3/D5 approach to black holes with KT asymptotics. For example, our solution does not allow for a generic mode that will lead to a Freund-Rubin form for $F_{5}$. As has been repeatedly emphasized, our $F_{5}$ is related to the non-extremality function and deforms the typical term proportional to the volume 5 -form. Let us explicitly visualize the difficulty in choosing the asymptotic expansion from the D3/D5 point of view in another example. The choice $C_{1}=0$ roughly corresponds to a dimension 3 operator in the D3/D5 picture. This can be seen easily by going to the radial coordinate $u=\exp (2 \rho / 3)$.

papers [10], [22] is such that the dilaton asymptotes to a (tunable) constant and the subleading terms are precisely those needed to generate the warp factor of Klebanov-Strassler 


\section{Conclusions and Future Directions}

We present here some concluding remarks together with some possible future projects to complement and refine the material in this paper.

In this work we have elaborated on the U-duality proposed in [6]. In that case, it was used to connect solutions describing wrapped D5 branes with the family of solutions describing the strong coupling dynamics of the baryonic branch of the Klebanov-Strassler field theory. In our case, we have used this U-duality to construct a new family of solutions describing the finite temperature phase of this baryonic branch. But before doing so, we explored the U-duality (that, following custom, we called rotation) in more generality, as a solution generating technique. We applied it to flat Dp branes in two different circumstances so that we appreciated the subtle differences making the choice of dualities in [6] so special.

Once we developed this technology, we applied it to rotate the Witten model for a Yang-Mills dual [17]. All this represents new material and it would be interesting to find an application for it, hopefully in the context of duals to Yang-Mills or QCD-like field theories.

In section 3, we discussed various new things: first a class of non-extremal solutions describing five branes wrapping a two-cycle inside the resolved conifold. The main feature of these non-extremal backgrounds is that the dilaton is asymptotically stabilized, which we interpreted as coupling the dual field theory to gravity modes. Then, we applied the U-duality to this solution, to find a new background of IIB that describes a non-extremal deformation of the Klebanov-Tseytlin solution (decoupled from gravity or stringy modes). We briefly commented on the associated thermodynamic properties of this new family of backgrounds. Finally, we discussed various aspects of this coupling/decoupling from gravity modes in section 4 .

Let us now list a set of projects that complement the material presented here and that may improve the understanding of the topic:

- It is important to sort out the details of the thermodynamics after the rotation in the solutions presented in section 3. Indeed, as we indicated in section 3.5, this poses a numerical problem, but certainly does not amount to a conceptual obstruction. The result for quantities like the specific heat or the free energy will be of great interest. Notice that this presents a novel way to approach the finite temperature phase of the Klebanov-Strassler field theory. Comparison with the solutions of [38] would be interesting.

- More conceptually; it would be nice to better understand the role played by the D0 branes in this solution generating technique. Indeed, notice the differences between the examples analyzed in section 2 and the one in section 2.1, all due to the presence of D0 branes before the boost that translates into the different final results. In the same vein, it would be good to have a better understanding on the reasons for the equivalence between the U-duality explained here and the rotation of the $J_{2}, \Omega_{3}$ forms as described 
in $[12],[13]$.

- It would be of very much interest to extend our non-extremal solution to a background of the form in eq.(3.28) where the restriction $a(\rho)=b(\rho)=0$ is not applied. Indeed, finding such a non-extremal solution would, after the application of the U-duality, produce a solution dual to a non-SUSY KS-like field theory in the baryonic branch. It may prove interesting to compare this new solution with that presented in the papers [35]. The different nature of the non-SUSY deformation will certainly reflect in different field theory aspects.

- It would be nice to use the solution presented in section 3 as a base to produce a non-SUSY deformation along the line proposed recently in [36]. The authors of [36] are finding SUSY breaking backgrounds by adding anti-D3 branes. Their solutions have singularities (and the problem is if such a singularity is physically acceptable). Proceeding as with the solution in this paper may set us on a different branch of solutions.

- It would be interesting to find a Physics application of the new backgrounds of section 2.1.1.

- It would be nice to extend the treatment here to the case in which the seed background contains $N_{f} \sim N_{c}$ flavors. This would be following the lead of the papers [24], [25]. The subtle point would be how to rotate such solutions (in other words, how the sources get represented in M-theory, see [37]. Comparison with the solutions of [38] would be interesting)

- We have presented two numerical solutions as examples of the type of backgrounds obtained. These examples correspond to specific values of the UV parameteers. We did not attempt a study of the full family of solutions in the whole parameter space. This is an interesting question worth investigating.

We hope to address some of these problems in the near future.

\section{Acknowledgments:}

We would like to thank various colleagues for the input that allowed us to better understand and present the results of this paper. We would like to single out Eloy Ayón-Beato, Iosif Bena, Alex Buchel, Pau Figueras, Gregory Giecold, Mariana Graña, Prem Kumar, Dario Martelli, Ioannis Papadimitriou, Michela Petrini, Dori Reichmann and César Terrero-Escalante. Very special thanks to Gregory Giecold for a very careful reading of the manuscript. E.C. thanks the Theory Group at the University of Texas at Austin for hospitality. 
This work was supported in part by the National Science Foundation under Grant Numbers PHY-0969020 and PHY-0455649, the US Department of Energy under grant DE-FG0295ER40899 and CONACyT grant CB-2008-01-104649.

\section{A Appendix: U-duality in the case of flat Dp-branes}

In this appendix we will give a sketchy derivation of the U-duality chain, in the case in which we rotate flat Dp-branes. We will not consider the non-extremal case, this can be done easily and the result of doing so is included in the first section of the paper. Here we want just to emphasize some points.

We start from the SUSY configuration in type II supergravity (string frame),

$$
\begin{aligned}
& d s^{2}=H^{-1 / 2}\left(-d t^{2}+d x_{p}^{2}\right)+H^{1 / 2}\left(d \rho^{2}+\rho^{2} d \Omega_{8-p}\right), \\
& e^{2 \phi}=H^{\frac{3-p}{2}}, F_{p+2}=\partial_{\rho} \hat{A} d t \wedge d x_{1} \wedge \ldots d x_{p} \wedge d \rho
\end{aligned}
$$

where $\hat{A}=-A$ in the notation of Section 2. Following standard convention we define the charge of $F_{8-p}$ as positive. The sign of $F_{p+2}$ will then depend on whether we consider Minkowski or Euclidean worldvolume on the $D_{p}$ brane. We apply T-duality in the $x_{p}$ directions,

$$
\begin{aligned}
d s^{2} & =-H^{-1 / 2} d t^{2}+H^{1 / 2}\left(d x_{p}^{2}+d \rho^{2}+\rho^{2} d \Omega_{8-p}\right), \\
e^{2 \phi} & =H^{\frac{3}{2}}, \quad F_{2}=\partial_{\rho} \hat{A} d t \wedge d \rho
\end{aligned}
$$

We lift this to M-theory, using $e^{\frac{4 \phi}{3}}=H$,

$$
d s_{11}^{2}=H\left(d x_{11}+\hat{A} d t\right)^{2}-H^{-1} d t^{2}+d M_{9}, d M_{9}=\left(d x_{p}^{2}+d \rho^{2}+\rho^{2} d \Omega_{8-p}\right)
$$

we now boost in the $t-x_{11}$ direction with rapidity $\beta$

$$
d t \rightarrow \cosh \beta d t-\sinh \beta d x_{11}, \quad d x_{11} \rightarrow-\sinh \beta d t+\cosh \beta d x_{11},
$$

to get

$$
\begin{aligned}
& d s_{11}^{2}=A d t^{2}+B d x_{11}^{2}+C d t d x_{11}+d M_{9} \\
& A=H^{-1}\left[H^{2}(\hat{A} \cosh \beta-\sinh \beta)^{2}-\cosh ^{2} \beta\right] \\
& B=H^{-1}\left[H^{2}(\hat{A} \sinh \beta-\cosh \beta)^{2}-\sinh ^{2} \beta\right] \\
& C=-2 H^{-1}\left[H^{2}(\hat{A} \cosh \beta-\sinh \beta)(\hat{A} \sinh \beta-\cosh \beta)-\cosh \beta \sinh \beta\right]
\end{aligned}
$$

and we prepare the metric for reduction back to IIA as

$$
\begin{aligned}
& d s_{11}^{2}=B\left(d x_{11}-a_{t} d t\right)^{2}+B^{-1 / 2}\left[g_{t t} d t^{2}+B^{1 / 2} d M_{9}\right], \\
& a_{t}=-\frac{C}{2 B}, \quad g_{t t}=\frac{4 A B-C^{2}}{4 \sqrt{B}}
\end{aligned}
$$


we now write the IIA configuration as,

$$
d s_{I I A}^{2}=g_{t t} d t^{2}+B^{1 / 2}\left(d x_{p}^{2}+d \rho^{2}+\rho^{2} d \Omega_{8-p}\right), e^{2 \phi}=B^{3 / 2}, F_{2}=\partial_{\rho} a_{t} d t \wedge d \rho
$$

and we T-dualize back in the $x_{p}$ directions to get

$$
\begin{aligned}
& d s_{I I}^{2}=g_{t t} d t^{2}+B^{-1 / 2} d x_{p}^{2}+B^{1 / 2}\left(d \rho^{2}+\rho^{2} d \Omega_{8-p}\right), \\
& e^{2 \phi}=B^{\frac{3-p}{2}}, \quad F_{p+2}=\partial_{\rho} a_{t} d t \wedge d \rho \wedge d x_{1} \ldots \wedge d x_{p}
\end{aligned}
$$

We see that the $S O(1, p)$ invariance is preserved, as $g_{t t} \sqrt{B}=-1$.

\section{B Appendix: Black Dp branes}

In this appendix we gather some useful facts about black Dp-brane solutions [26],[27],[28]. The metric in string frame is,

$$
d s^{2}=-\frac{f_{+}(r)}{\sqrt{f_{-}(r)}} d t^{2}+\sqrt{f_{-}(r)} \sum_{i=1}^{p} d x^{i} d x^{i}+\frac{f_{-}(r)^{-\frac{1}{2}-\frac{5-p}{7-p}}}{f_{+}(r)} d r^{2}+r^{2} f_{-}(r)^{\frac{1}{2}-\frac{5-p}{7-p}} d \Omega_{8-p}^{2}
$$

where

$$
f_{ \pm}(r)=1-\left(\frac{r_{ \pm}}{r}\right)^{7-p}
$$

The mass per unit volume $M$ and the R-R charge $N$ are

$$
M=\frac{1}{(7-p)(2 \pi)^{7} d_{p} l_{P}^{8}}\left((8-p) r_{+}^{7-p}-r_{-}^{7-p}\right), \quad N=c_{p}\left(r_{+} r_{-}\right)^{\frac{7-p}{2}},
$$

where $l_{P}$ is the ten dimensional Planck length $l_{P}=g_{s}^{1 / 4} l_{s}$ and $c_{p}, d_{p}$ are numerical factors,

$$
\begin{aligned}
& d_{p}=2^{5-p} \pi^{\frac{5-p}{2}} \Gamma\left(\frac{7-p}{2}\right) \\
& c_{p}=\frac{1}{d_{p} g_{s} l_{s}^{7-p}}=\frac{(7-p) V o l_{S^{8-p}}}{\left(2 \pi l_{s}\right)^{7-p} g_{s}}
\end{aligned}
$$

The background also has a nontrivial dilaton and a R-R flux,

$$
\begin{aligned}
e^{-2 \Phi} & =g_{s}^{-2} f_{-}(r)^{-\frac{p-3}{2}} \\
\frac{1}{\left(2 \pi l_{s}\right)^{7-p}} \int_{S^{8-p}} * F_{p+2} & =N .
\end{aligned}
$$

The Einstein frame metric $\left(g_{E_{\mu \nu}}=\sqrt{g_{s} e^{-\Phi}} g_{s t_{\mu \nu}}\right)$ has a horizon at $r=r_{+}$and, for $p \leq 6$, a singularity at $r=r_{-}$. If $r_{+}>r_{-}$the singularity is covered by the horizon and the solution is a black hole. In the extremal case, $r_{+}=r_{-}$, the space is singular except for $p=3$. 
Let us define a new coordinate,

$$
\rho^{7-p}=r^{7-p}-r_{-}^{7-p}
$$

This change of variables transforms (B.1) to a more familiar form,

$$
d s^{2}=H(\rho)^{-\frac{1}{2}}\left(-h(\rho) d t^{2}+\sum_{i=1}^{p} d x^{i} d x^{i}\right)+H(\rho)^{\frac{1}{2}}\left(\frac{d \rho^{2}}{h(\rho)}+\rho^{2} d \Omega_{8-p}^{2}\right)
$$

where

$$
\begin{aligned}
& H(\rho)=1+\frac{r_{-}^{7-p}}{\rho^{7-p}} \\
& h(\rho)=1-\frac{r_{+}^{7-p}-r_{-}^{7-p}}{\rho^{7-p}}
\end{aligned}
$$

Throughout the paper we use the notation,

$$
L_{p}^{7-p} \equiv r_{-}^{7-p} \quad \text { and } \quad R_{T}^{7-p} \equiv r_{+}^{7-p}-r_{-}^{7-p}
$$

Note that using (B.3) one can also write

$$
\begin{aligned}
L_{p}^{7-p} & =\tilde{Q}\left[\sqrt{1+\left(\frac{R_{T}^{7-p}}{2 \tilde{Q}}\right)^{2}}-\frac{R_{T}^{7-p}}{2 \tilde{Q}}\right] \\
& =\tilde{Q} \alpha_{p} .
\end{aligned}
$$

where $\tilde{Q}=\frac{N}{c_{p}}$ and $\alpha_{p}=\left(1+\left(\frac{R_{T}^{7-p}}{2 \tilde{Q}}\right)^{2}\right)^{1 / 2}-\frac{R_{T}^{7-p}}{2 \tilde{Q}}$.

¿From (B.3) we can see that the parameter $R_{T}$ is related to the energy per unit volume above extremality $\epsilon$,

$$
\Delta M=\epsilon=\frac{r_{+}^{7-p}-r_{-}^{7-p}}{(2 \pi)^{7} d_{p} l_{P}^{8}}=\frac{R_{T}{ }^{7-p}}{(2 \pi)^{7} d_{p} l_{P}^{8}} .
$$

Note that in the decoupling limit $\left(\alpha^{\prime} \rightarrow 0\right.$, energies fixed $) \epsilon$ remains fixed and $\alpha_{p} \rightarrow 1$.

In the extremal case, $r_{+}=r_{-}$, we have $R_{T}=0$ and $L_{p}^{7-p}=\tilde{Q}=N d_{p} g_{s} l_{s}^{7-p}$.

\section{Appendix: Some details on the U-duality}

Here we provide details for the derivations of eq.(2.24). We started with

$$
\begin{aligned}
& d s^{2}=H^{-1 / 2}\left[-d t^{2}+d x_{p-q}^{2}+d \sigma_{q}^{2}\right]+H^{1 / 2}\left[d \rho^{2}+\rho^{2} d \Omega_{8-p}^{2}\right] \\
& F_{p+2}=\partial_{\rho} \hat{A} d t \wedge d x_{1} \wedge \ldots \wedge d x_{p-q} \wedge d \sigma_{q} \wedge d \rho, \\
& e^{2 \phi[\text { initial }]}=e^{2 \phi(\infty)} H^{\frac{3-p}{2}} .
\end{aligned}
$$


The we perform the U-duality described in the text below eq.(2.23)

$$
\begin{aligned}
& d s^{2}=H^{-1 / 2}\left[-d t^{2}+d \sigma_{q}^{2}\right]+H^{1 / 2}\left[d \rho^{2}+\rho^{2} d \Omega_{8-p}^{2}+d x_{p-q}^{2}\right] \\
& F_{q+2}=\partial_{r} \hat{A} d t \wedge d \sigma_{q} \wedge d \rho \\
& e^{2 \phi}=e^{2 \phi(\infty)} H^{\frac{3-q}{2}}
\end{aligned}
$$

Now, we need this to be a configuration in Type IIA (in order to lift this to M-theory). We will also want to impose that when lifted to M-theory this will produce a four-form field $G_{4}$. If this is the case, we must have that either $q=2$ or that $q=4$ (the cases of $q=0, q=6$ are analogous to what we analyzed in the first section). The case $q=2$, on which we will elaborate upon below, has the peculiarity that the reduction from eleven dimensions back to IIA will generate a NS $H_{3}$ field, proportional to the boost. Let us see this in detail. To begin with, we will lift to eleven dimensions the configuration in eq.(C.2).

$$
\begin{aligned}
& d s_{11}^{2}=H^{1 / 3} d x_{11}^{2}+H^{-2 / 3}\left[-d t^{2}+d \vec{\sigma}_{2}^{2}\right]+H^{1 / 3}\left[d \rho^{2}+\rho^{2} d \Omega_{8-p}^{2}+d \vec{x}_{p-2}^{2}\right] \\
& G_{4}=\partial_{\rho} \hat{A} d t \wedge d \rho \wedge d \sigma_{1} \wedge d \sigma_{2}
\end{aligned}
$$

and now we boost with rapidity $\beta$

$$
d t \rightarrow \cosh \beta d t-\sinh \beta d x_{11}, \quad d x_{11} \rightarrow-\sinh \beta d t+\cosh \beta d x_{11}
$$

and we can rewrite the configuration after the boost as,

$$
\begin{aligned}
& d s_{11}^{2}=A d t^{2}+B d x_{11}^{2}+C d t d x_{11}+d M_{9}^{2}, \\
& G_{4}=\partial_{\rho} \hat{A}\left(\cosh \beta d t-\sinh \beta d x_{11}\right) \wedge d \rho \wedge d \sigma_{1} \wedge d \sigma_{2}, \\
& d M_{9}^{2}=H^{-2 / 3} d \vec{\sigma}_{2}^{2}+H^{1 / 3}\left[d \rho^{2}+\rho^{2} d \Omega_{8-p}^{2}+d \vec{x}_{p-2}^{2}\right], \\
& A=H^{-2 / 3}\left[H \sinh ^{2} \beta-\cosh ^{2} \beta\right] \\
& B=H^{-2 / 3}\left[H \cosh ^{2} \beta-\sinh ^{2} \beta\right] \\
& C=2 H^{-2 / 3} \sinh \beta \cosh \beta(1-H)
\end{aligned}
$$

when we reduce this to IIA we get,

$$
\begin{aligned}
& d s_{I I A, s t}^{2}=g_{t t} d t^{2}+B^{1 / 2}\left(H^{-2 / 3} d \vec{\sigma}_{2}^{2}+H^{1 / 3}\left[d \rho^{2}+\rho^{2} d \Omega_{8-p}^{2}+d \vec{x}_{p-2}^{2}\right]\right) \\
& e^{2 \phi[A]}=B^{3 / 2}, \quad g_{t t}=\frac{4 A B-C^{2}}{4 \sqrt{B}} \\
& F_{4}=\partial_{\rho} \hat{A}\left(\cosh \beta+a_{t} \sinh \beta\right) d t \wedge d \rho \wedge d \sigma_{1} \wedge d \sigma_{2} \\
& H_{3}=\sinh \beta \partial_{\rho} \hat{A} d \rho \wedge d \sigma_{1} \wedge d \sigma_{2}, \\
& F_{2}=\partial_{\rho}\left(a_{t}\right) d t \wedge d \rho, \quad a_{t}=\frac{C}{2 B}=\frac{\sinh \beta \cosh \beta(1-H)}{H \cosh \beta-\sinh ^{2} \beta}
\end{aligned}
$$


we see that we have generated a NS magnetic field. Finally, we T-dualize back in the $\vec{x}_{p-1}$ directions, to get

$$
\begin{aligned}
& d s_{I I, s t}^{2}=g_{t t} d t^{2}+\frac{d \vec{x}_{p-2}^{2}}{H^{1 / 3} B^{1 / 2}}+B^{1 / 2}\left(H^{-2 / 3} d \vec{\sigma}_{2}^{2}+H^{1 / 3}\left[d \rho^{2}+\rho^{2} d \Omega_{8-p}^{2}\right]\right), \\
& F_{p+2}=\partial_{\rho} \hat{A}\left(\cosh \beta+a_{t} \sinh \beta\right) \wedge d \rho \wedge d \sigma_{1} \wedge d \sigma_{2} \wedge d x_{1} \wedge \ldots \wedge d x_{p-2}, \\
& H_{3}=\sinh \beta \partial_{\rho} \hat{A} d \rho \wedge d \sigma_{1} \wedge d \sigma_{2}, \\
& F_{p}=\partial_{\rho}\left(a_{t}\right) d t \wedge d \rho \wedge d x_{1} \wedge \ldots \wedge d x_{p-2}, \\
& e^{2 \phi[\text { final }]}=B^{\frac{5-p}{2}} H^{\frac{2-p}{3}} .
\end{aligned}
$$

This completes our derivation of eq.(2.24).

\section{Appendix: Another solution generating algorithm}

We studied two different 'solution generating techniques' and applied them to different backgrounds. All these 'algorithms' were starting with a background solution to the Type II (A or B) equations of motion, applying a number of T-dualities that would transform the background into a solution for Type IIA supergravity. Then we lifted this to eleven dimensions, where a boost was applied (inducing a one parameter- $\beta$ - family of solutions), then reducing to IIA and T-dualizing back we had our final generated background.

One may wonder what is the algorithm when, after a number of T-dualities, we end with a background solving the Type IIB supergravity equations of motion. In this case, a way of generating a one-parameter family of solutions may be S-dualizing. Indeed, given $a, b, c, d$ real numbers satisfying $a d-b c=1$, we can start with a IIB solution having axion $\chi$, dilaton $\phi, \mathrm{RR}$ and NS thre forms $F_{3}=d C_{2}$ and $H_{3}=d B_{2}$ and five form $F_{5}=d C_{4}+F_{3} \wedge B_{2}$ and by S-duality, generate a new solutions dependent on the three independent real parameters $a, b, c$. Indeed, the five form is left invariant and the same happens for the Einstein frame metric, while

$$
\begin{aligned}
& F_{3}[\text { new }]=b H_{3}+a F_{3}, \quad H_{3}[\text { new }]=d H_{3}+c F_{3}, \\
& e^{\phi[n e w]}=\left((c \chi+d)^{2}+c^{2} e^{-2 \phi}\right) e^{\phi}, \quad \chi[n e w]=\frac{(a \chi+b)(c \chi+d)+a c e^{-2 \phi}}{\left((c \chi+d)^{2}+c^{2} e^{-2 \phi}\right)}
\end{aligned}
$$

Let us focus our attention in the particular set of values $a=d=0, c b=-1$, this is the transformation that interchanges the three forms and inverts the value of the dilaton (if the axion is initially zero, as we will assume).

One can then think about applying this transformation expecting to generate new interesting solutions. As an example, suppose that we start with solutions describing D6 branes wrapping a three-cycle inside the deformed conifold. Those solutions are dual to a (UV completed version of) $\mathrm{N}=1 \mathrm{SYM}$ [29]. A particularly interesting solution is given in section 3.2 of 
the paper [30]. The background consist of metric, dilaton and RR two-form field,

$$
d s_{I I A, s t}^{2}=e^{f}\left[-d t^{2}+d x_{1}^{2}+d x_{2}^{2}+d x_{3}^{2}+d M_{6}^{2}\right], \quad e^{2 \phi}=e^{3 f+2 \phi_{0}}, \quad F_{2}=d A_{1}
$$

where all the details (the manifold $M_{6}$, the functional form of $f, \phi$ and the one form $A_{1}$ are given in eqs(57)-(59) of [30]). We can the perform three T-dualities in $x_{1,2,3}$ leading to

$$
\begin{aligned}
& d s_{I I B, s t}^{2}=e^{f}\left[-d t^{2}+d M_{6}^{2}\right]+e^{-f} d x_{1,2,3}^{2}, \quad e^{2 \phi}=e^{2 \phi_{0}}, \\
& F_{5}=d A_{1} \wedge d x_{1} \wedge d x_{2} \wedge d x_{3}
\end{aligned}
$$

that is we have generated D3 brane charge and the dilaton is just a constant. We now move this to Einstein frame, that leaves us-up to a constant-with the same metric, perform the Sduality metioned above and T-dualize back. This brings us to the starting point background $(D .2)^{10}$. Something similar occurs if we start with a solution describing D5 branes wrapping a three-cycle inside a G2 holonomy manifold [31], a configuration dual to N=1 Yang-Mills ChernSimons in $2+1$ dimensions (with its respective UV completion). Notice that this operation is not the one proposed in [20], that is the reason why these authors were able to generate an interesting solution starting from [31].

In both these cases described above, we are generating charge of D3 brane, this is invariant under the S-duality, hence it is expected that the whole operation brings us back to the initial configuration. A more interesting example is to start from the configuration of D4 branes wrapping a circle with SUSY breaking boundary conditions [17] that we discussed before, see Section 2.1.1.

In this case we will apply three T-dualities to a IIA configuration. This will generate charge of D1 brane that the S-duality will interchange with F1 charge, after T-dualizing back, we will generate a new background (the $S O(1,3)$ isometry will be spoiled, so we may want to start with the high Temperature dual, hence having a non-extremal factor $h(\rho)$ in front of $d t^{2}$ and the function $f(\rho)=1$ in front of $d x_{4}^{2}$, but let us keep things general). Let us see some details. After the T-dualities, the configuration reads

$$
\begin{aligned}
& d s_{I I B, s t}^{2}=H^{-1 / 2}\left(-h d t^{2}+f d x_{4}^{2}\right)+H^{1 / 2}\left(\frac{d \rho^{2}}{h}+\rho^{2} d \Omega_{4}^{2}+d x_{1,2,3}^{2}\right), \\
& e^{2 \phi}=g_{s}^{2} H, \quad F_{3}=\partial_{\rho} A d t \wedge d x_{4} \wedge d \rho
\end{aligned}
$$

now, we need to move this to Einstein frame, multiplying the metric by $e^{-\frac{\phi}{2}}$, perform the S-duality that will generate $H_{3}$ and a dilaton $e^{-2 \phi}=g_{s}^{2} H$. Then, T-dualize back in $x_{1,2,3}$. The final configurations is,

$$
\begin{aligned}
& d s_{I I A, s t}^{2}=\frac{c^{1 / 4}}{g_{s} H}\left(-h d t^{2}+f d x_{4}^{2}\right)+\frac{d \rho^{2}}{h}+\rho^{2} d \Omega_{4}^{2}+d x_{1,2,3}^{2}, \\
& H_{3}=c \partial_{\rho} A d t \wedge d x_{4} \wedge d \rho, \quad c^{4} e^{-2 \phi}=g_{s}^{2} H
\end{aligned}
$$

\footnotetext{
${ }^{10}$ One may perform an S-duality with parameters $a=0, b c=-1$ and $d$ being free. This will generate a background in IIB with constant axion-dilaton. The final IIA configuration is the same as the initial one.
} 
As a final remark; had we chosen to T-dualize only in the $x_{4}$ direction (in the supergravity approximation, this can be done in the high Temperature phase only), we would have generated D3 branes, hence after the S-duality and T-duality, we would be back to the initial configuration.

\section{E Appendix: U-duality for the wrapped D5 branes Black Hole}

We will describe in detail the action of the solution generating technique proposed in [6], when applied to the background of eq.(3.30). These techniques have been applied in a similar context in [39]. Let us consider things in the string frame.

$$
\begin{aligned}
& d s_{s}^{2}=e^{\phi}\left[-h(\rho) d t^{2}+d x_{1}^{2}+d x_{2}^{2}+d x_{3}^{2}\right]+d s_{6, s}^{2} \\
& d s_{6}^{2}=e^{\phi}\left[\frac{e^{2 k}}{s(\rho)} d \rho^{2}+\frac{e^{2 k}}{4}\left(\tilde{\omega}_{3}+\cos \theta d \varphi\right)^{2}+e^{2 q}\left(d \theta^{2}+\sin ^{2} \theta d \varphi^{2}\right)+\frac{e^{2 g}}{4}\left(d \tilde{\theta}^{2}+\sin ^{2} \tilde{\theta} d \tilde{\varphi}^{2}\right)\right] \\
& F_{(3)}=\frac{N_{c}}{4}\left[-\tilde{\omega}_{1} \wedge \tilde{\omega}_{2}+\sin \theta d \theta \wedge d \varphi\right] \wedge\left(\tilde{\omega}_{3}+\cos \theta d \varphi\right)
\end{aligned}
$$

where $h(\rho), s(\rho)$ are the non-extremality functions and we will choose $h(\rho)=s(\rho)$ as we did in section 3. We can proceed to rotate it. We will follow the procedure explained in [6]. So, let us start by writing the effect of the first T-duality in the $x_{1}$ direction (all the expressions below are in string frame)

$$
\begin{aligned}
& d s_{I I A}^{2}=e^{f}\left[-h d t^{2}+d x_{2}^{2}+d x_{3}^{2}\right]+e^{-f} d x_{1}^{2}+d s_{6}^{2}, \\
& e^{2 \phi_{A}}=e^{2 \phi-f}, \quad F_{4}=F_{3} \wedge d x_{1}
\end{aligned}
$$

The function $f=\phi$ will be kept to avoid confusion with the transformed dilatons. Now, we perform the T-duality in $x_{2}$

$$
\begin{aligned}
& d s_{I I A}^{B}=e^{f}\left[-h d t^{2}+d x_{3}^{2}\right]+e^{-f}\left(d x_{1}^{2}+d x_{2}^{2}\right)+d s_{6}^{2}, \\
& e^{2 \phi_{B}}=e^{2 \phi-2 f}, \quad F_{5}=F_{3} \wedge d x_{1} \wedge d x_{2}\left(1+*_{10}\right)
\end{aligned}
$$

T-dualizing in $x_{3}$, we get

$$
\begin{aligned}
& d s_{I I A}^{2}=e^{f}\left[-h d t^{2}\right]+e^{-f}\left(d x_{1}^{2}+d x_{2}^{2}+d x_{3}^{2}\right)+d s_{6}^{2}, \\
& e^{2 \phi_{A}}=e^{2 \phi-3 f}, \\
& F_{6}=F_{3} \wedge d x_{1} \wedge d x_{2} \wedge d x_{3} \rightarrow F_{4}=e^{2 f} \sqrt{h} *_{6} F_{3} \wedge d t
\end{aligned}
$$


Notice that

$$
*_{6} F_{3}=\frac{N_{c}}{\sqrt{h}}\left[-2 e^{2 q-2 g} \sin \theta d \theta \wedge d \varphi+\frac{e^{2 g-2 q}}{8} \sin \tilde{\theta} d \tilde{\theta} \wedge d \tilde{\varphi}\right] \wedge d \rho .
$$

The factor of $\sqrt{h}$ in the $F_{4}$ of eq.(E.4) is present to cancel the factor of $\sqrt{h}$ in the denominator of eq.(E.5). Now, we lift this to M-theory;

$$
\begin{aligned}
& d s_{11}^{2}=e^{4 / 3 \phi-2 f} d x_{11}^{2}+e^{f-2 / 3 \phi}\left[-h e^{f} d t^{2}+e^{-f}\left(d x_{1}^{2}+d x_{2}^{2}+d x_{3}^{2}\right)+d s_{6}^{2}\right], \\
& G_{4}=\sqrt{h} e^{2 f} *_{6} F_{3} \wedge d t .
\end{aligned}
$$

We boost in the $t-x_{11}$ directions according to,

$$
d t \rightarrow \cosh \beta d t-\sinh \beta d x_{11}, \quad d x_{11} \rightarrow-\sinh \beta d t+\cosh \beta d x_{11}
$$

and now we rewrite this boosted metric as,

$$
\begin{aligned}
& d s_{11}^{2}=e^{f-2 / 3 \phi}\left[e^{-f}\left(d x_{1}^{2}+d x_{2}^{2}+d x_{3}^{2}\right)+d s_{6}^{2}\right]+A d t^{2}+B d x_{11}^{2}+C d t d x_{11}, \\
& G_{4}=\sqrt{h} e^{2 f} *_{6} F_{3}\left[\cosh \beta d t-\sinh \beta d x_{11}\right]
\end{aligned}
$$

where,

$$
\begin{aligned}
& A=e^{2 f-2 / 3 \phi}\left[\sinh ^{2} \beta e^{2 \phi-4 f}-h \cosh ^{2} \beta\right], \quad B=e^{2 f-2 / 3 \phi}\left[\cosh ^{2} \beta e^{2 \phi-4 f}-h \sinh ^{2} \beta\right], \\
& C=-2 \cosh \beta \sinh \beta e^{2 f-2 / 3 \phi}\left[e^{2 \phi-4 f}-h\right] .
\end{aligned}
$$

Now, we will reduce this to IIA, before doing so and in order to reduce to IIA, it is useful to rewrite eq.(E.8) as,

$$
\begin{aligned}
& d s_{11}^{2}=B^{-1 / 2}\left[g_{t t} d t^{2}+B^{1 / 2} e^{f-2 / 3 \phi}\left(e^{-f}\left(d x_{1}^{2}+d x_{2}^{2}+d x_{3}^{2}\right)+d s_{6}^{2}\right)\right]+B\left(d x_{11}+a_{t} d t\right)^{2}, \\
& G_{4}=\sqrt{h} e^{2 f} *_{6} F_{3}\left[\left(\cosh \beta+a_{t} \sinh \beta\right) d t-\sinh \beta\left(d x_{11}+a_{t} d t\right)\right]
\end{aligned}
$$

where we have defined

$$
a_{t}=\frac{C}{2 B}, \quad g_{t t}=\frac{4 A B-C^{2}}{4 \sqrt{B}}, \quad e^{4 / 3 \phi_{A}}=B .
$$

Now, we reduce to IIA, obtaining in string frame,

$$
\begin{aligned}
& d s_{I I A}^{2}=g_{t t} d t^{2}+\sqrt{B} e^{-2 / 3 \phi}\left(d x_{1}^{2}+d x_{2}^{2}+d x_{3}^{2}\right)+\sqrt{B} e^{f-2 / 3 \phi} d s_{6}^{2}, \\
& e^{2 \phi_{A}}=B^{3 / 2}, \\
& F_{4}=\sqrt{h} e^{2 f} *_{6} F_{3} \wedge\left[\left(\cosh \beta+a_{t} \sinh \beta\right) d t\right], \\
& H_{3}=-\sinh \beta \sqrt{h} e^{2 f} *_{6} F_{3}, \\
& F_{2}=a_{t}^{\prime} d \rho \wedge d t
\end{aligned}
$$


Now, we proceed to do the T-dualities back; T-dualizing in the $x_{3}$ direction we have

$$
\begin{aligned}
& d s_{I I B}^{2}=g_{t t} d t^{2}+\sqrt{B} e^{-2 / 3 \phi}\left(d x_{1}^{2}+d x_{2}^{2}\right)+\frac{e^{2 / 3 \phi}}{\sqrt{B}} d x_{3}^{2}+\sqrt{B} e^{f-2 / 3 \phi} d s_{6}^{2}, \\
& e^{2 \phi_{B}}=B e^{2 / 3 \phi}, \\
& F_{5}=\sqrt{h} e^{2 f} *_{6} F_{3} \wedge\left[\left(\cosh \beta+a_{t} \sinh \beta\right) d t \wedge d x_{3}\right]\left(1+*_{10}\right), \\
& H_{3}=-\sinh \beta \sqrt{h} e^{2 f} *_{6} F_{3}, \\
& F_{3}=a_{t}^{\prime} d \rho \wedge d t \wedge d x_{3}
\end{aligned}
$$

now, we T-dualize in $x_{2}$

$$
\begin{aligned}
& d s_{I I A}^{2}=g_{t t} d t^{2}+\sqrt{B} e^{-2 / 3 \phi}\left(d x_{1}^{2}\right)+\frac{e^{2 / 3 \phi}}{\sqrt{B}}\left(d x_{3}^{2}+d x_{2}^{2}\right)+\sqrt{B} e^{f-2 / 3 \phi} d s_{6}^{2}, \\
& e^{2 \phi_{A}}=\sqrt{B} e^{4 / 3 \phi}, \\
& F_{6}=\sqrt{h} e^{2 f} *_{6} F_{3} \wedge\left[\left(\cosh \beta+a_{t} \sinh \beta\right) d t\right] \wedge d x_{3} \wedge d x_{2}, \\
& H_{3}=-\sinh \beta \sqrt{h} e^{2 f} *_{6} F_{3}, \\
& F_{4}=a_{t}^{\prime} d \rho \wedge d t \wedge d x_{3} \wedge d x_{2}
\end{aligned}
$$

finally, we T-dualize in $x_{1}$

$$
\begin{aligned}
& d s_{I I B}^{2}=g_{t t} d t^{2}+\frac{e^{2 / 3 \phi}}{\sqrt{B}}\left(d x_{3}^{2}+d x_{1}^{2}+d x_{2}^{2}\right)+\sqrt{B} e^{f-2 / 3 \phi} d s_{6}^{2} \\
& e^{2 \phi_{B}}=e^{2 \phi} \\
& F_{7}=\sqrt{h} e^{2 f} *_{6} F_{3} \wedge\left[\left(\cosh \beta+a_{t} \sinh \beta\right) d t\right] \wedge d x_{3} \wedge d x_{2} \wedge d x_{1} \quad F_{3}=*_{10} F_{7}, \\
& H_{3}=-\sinh \beta \sqrt{h} e^{2 f} *_{6} F_{3}, \\
& F_{5}=a_{t}^{\prime} d \rho \wedge d t \wedge d x_{3} \wedge d x_{2} \wedge d x_{1}\left(1+*_{10}\right) .
\end{aligned}
$$

After using $f=\phi$ and the definitions for $A, B, C, a_{t}$ this encodes the result of eq.(3.35).

\section{F Appendix: The equations of motion}

In this appendix we will quote the equations of motion that we are numerically solving. Our goal is to find a black hole of the metrics described in the main text. In [23] the authors studied a very general Ansatz for non-extremal deformations of NS5 branes wrapped on $S^{2}$. Their Ansatz can be adapted to our case. It reads -in Einstein frame,

$$
d s^{2}=-Y_{1} d t^{2}+Y_{2} d \mathrm{x}^{n} d \mathrm{x}^{n}+Y_{3} d \rho^{2}+Y_{4}\left(e_{1}^{2}+e_{2}^{2}\right)+Y_{5}\left(\left(w^{1}\right)^{2}+\left(w^{2}\right)^{2}\right)+Y_{6}\left(w^{3}+A\right)^{2}
$$


Inserting this ansatz in the supergravity action we get,

$$
L=\sum_{i, j} G_{i j}(Y) Y_{i}^{\prime} Y_{j}^{\prime}-U(Y)=T-U
$$

Using a parametrization to make $G_{i j}$ diagonal and choosing the appropiate gauge to make contact with our ansatz we have,

$$
\begin{aligned}
T & =e^{2(g+q-4 x+\Phi)} \frac{1}{8}\left(\frac{1}{2}\left(g^{\prime 2}+q^{\prime 2}-2 \Phi^{\prime 2}\right)+2\left(g^{\prime} q^{\prime}-k^{\prime} x^{\prime}\right)+\left(g^{\prime}+q^{\prime}+\Phi^{\prime}\right)\left(k^{\prime}-4 x^{\prime}+2 \Phi^{\prime}\right)\right) \\
U & =\frac{1}{256} e^{-2(g+q-\Phi)}\left(-16 e^{2(g+q+k)}\left(e^{2 g}+4 e^{2 q}\right)+\left(e^{4 g}+16 e^{4 q}\right)\left(1+e^{4 k}\right)\right) .
\end{aligned}
$$

and,

$$
\begin{array}{lcc}
Y_{1}=e^{\Phi / 2} e^{-8 x}, & Y_{2}=e^{\Phi / 2}, & Y_{3}=e^{\Phi / 2} e^{8 x} e^{2 k}, \\
Y_{4}=e^{\Phi / 2} e^{2 g}, & Y_{5}=e^{\Phi / 2} e^{2 q} . & Y_{6}=\Phi
\end{array}
$$

¿From (F.2) we get the second order equations to solve,

$$
\begin{aligned}
& 2 e^{-4 g+8 x}+\frac{1}{8} e^{-4 q+8 x}-2 \Phi^{\prime}\left(g^{\prime}+q^{\prime}-4 x^{\prime}+\Phi^{\prime}\right)-\Phi^{\prime \prime}=0 \\
& -2 x^{\prime}\left(g^{\prime}+q^{\prime}-4 x^{\prime}+\Phi^{\prime}\right)-x^{\prime \prime}=0 \\
& -e^{4 k+8 x}\left(2 e^{-4 g}+\frac{1}{8} e^{-4 q}\right)+e^{8 x}\left(2 e^{-4 g}+\frac{1}{8} e^{-4 q}\right)+2 k^{\prime}\left(g^{\prime}+q^{\prime}-4 x^{\prime}+\Phi^{\prime}\right)+k^{\prime \prime}=0 \\
& 2 e^{8 x}\left(e^{4 k-4 g}-2 e^{2(k-g)}+1\right)+g^{\prime}\left(g^{\prime}+q^{\prime}-4 x^{\prime}+\Phi^{\prime}\right)+g^{\prime \prime}=0 \\
& e^{8 x}\left(-\frac{1}{8} e^{4 k-4 q}+e^{2 k-2 q}-\frac{1}{8} e^{-4 q}\right)-2 q^{\prime}\left(g^{\prime}+q^{\prime}-4 x^{\prime}+\Phi^{\prime}\right)-q^{\prime \prime}=0
\end{aligned}
$$

and a first order constraint which is a consequence of reparametrization invariance,

$$
\begin{aligned}
& \frac{e^{8 x}}{2}\left(\frac{1}{16}\left(e^{-4 q}+16 e^{-4 g}\right)\left(1+e^{4 k}\right)-e^{2 k}\left(e^{-2 q}+4 e^{-2 g}\right)\right)+\frac{1}{2}\left(g^{\prime 2}+q^{2}-2 \Phi^{\prime 2}\right)+ \\
& 2\left(g^{\prime} q^{\prime}-k^{\prime} x^{\prime}\right)+\left(g^{\prime}+q^{\prime}+\Phi^{\prime}\right)\left(k^{\prime}-4 x^{\prime}+2 \Phi^{\prime}\right)=0
\end{aligned}
$$

The second equation in (F.5) can be integrated to yield a first order equation and (F.5) becomes

$$
\begin{aligned}
2 e^{-4 g+8 x}+\frac{1}{8} e^{-4 q+8 x}-2 \Phi^{\prime}\left(g^{\prime}+q^{\prime}-4 x^{\prime}+\Phi^{\prime}\right)-\Phi^{\prime \prime} & =0 \\
x^{\prime}-\alpha e^{-2(g+q-4 x+\Phi)} & =0 \\
-e^{4 k+8 x}\left(2 e^{-4 g}+\frac{1}{8} e^{-4 q}\right)+e^{8 x}\left(2 e^{-4 g}+\frac{1}{8} e^{-4 q}\right)+2 k^{\prime}\left(g^{\prime}+q^{\prime}-4 x^{\prime}+\Phi^{\prime}\right)+k^{\prime \prime} & =0 \\
e^{8 x}\left(e^{4 k-4 g}-2 e^{2(k-g)}+1\right)+g^{\prime}\left(g^{\prime}+q^{\prime}-4 x^{\prime}+\Phi^{\prime}\right)+g^{\prime \prime} & =0 \\
e^{8 x}\left(-\frac{1}{8} e^{4 k-4 q}+e^{2 k-2 q}-\frac{1}{8} e^{-4 q}\right)-2 q^{\prime}\left(g^{\prime}+q^{\prime}-4 x^{\prime}+\Phi^{\prime}\right)-q^{\prime \prime} & =0
\end{aligned}
$$


where $\alpha$ is a non-extremality parameter.

One can verify that the transformation

$$
r \rightarrow e^{2 d} r \quad \Phi \rightarrow \Phi+d \quad x \rightarrow x+\frac{d}{2} \quad g \rightarrow g \quad q \rightarrow q \quad k \rightarrow k
$$

where $d$ is a constant, leaves (F.5) and (F.6) invariant and thus, is a symmetry of the equations of motion and constraint. Another, obvious, symmetry of (F.5) and ( F.6) is

$$
\Phi \rightarrow \Phi+C \quad \alpha \rightarrow \alpha e^{2 C}
$$

with all the other functions unchanged and $C$ a constant. Finally, there is also a translational symmetry

$$
r \rightarrow r+r_{0}
$$

that leaves all equations unchanged. Note that thermodynamic quantities should be invariant under all these symmetries.

\section{References}

[1] J. M. Maldacena, Adv. Theor. Math. Phys. 2, 231 (1998) [Int. J. Theor. Phys. 38, 1113 (1999)] [arXiv:hep-th/9711200].

[2] S. S. Gubser, I. R. Klebanov and A. M. Polyakov, Phys. Lett. B 428, 105 (1998) [arXiv:hep-th/9802109]. E. Witten, Adv. Theor. Math. Phys. 2, 253 (1998) [arXiv:hepth/9802150]. N. Itzhaki, J. M. Maldacena, J. Sonnenschein and S. Yankielowicz, Phys. Rev. D 58, 046004 (1998) [arXiv:hep-th/9802042].

[3] O. Lunin and J. M. Maldacena, JHEP 0505, 033 (2005) [arXiv:hep-th/0502086]. J. Maldacena, D. Martelli and Y. Tachikawa, JHEP 0810, 072 (2008) [arXiv:0807.1100 [hep-th]]. C. P. Herzog, M. Rangamani and S. F. Ross, JHEP 0811, 080 (2008) [arXiv:0807.1099 [hep-th]]. C. P. Herzog, M. Rangamani and S. F. Ross, JHEP 0811, 080 (2008) [arXiv:0807.1099 [hep-th]].

[4] V. Belinski and E. Verdaguer, Cambridge, UK: Univ. Pr. (2001) 258 pA. A. Pomeransky, Phys. Rev. D 73, 044004 (2006) [arXiv:hep-th/0507250]. T. Mishima and H. Iguchi, Phys. Rev. D 73, 044030 (2006) [arXiv:hep-th/0504018].

[5] H. Elvang and P. Figueras, JHEP 0705, 050 (2007) [arXiv:hep-th/0701035].

[6] J. Maldacena and D. Martelli, JHEP 1001, 104 (2010) [arXiv:0906.0591 [hep-th]].

[7] A. Butti, M. Grana, R. Minasian, M. Petrini and A. Zaffaroni, JHEP 0503, 069 (2005) [arXiv:hep-th/0412187]. 
[8] I. R. Klebanov and M. J. Strassler, JHEP 0008, 052 (2000) [arXiv:hep-th/0007191].

[9] R. Casero, C. Nunez and A. Paredes, Phys. Rev. D 73, 086005 (2006) [arXiv:hepth/0602027]. R. Casero, C. Nunez and A. Paredes, Phys. Rev. D 77, 046003 (2008) [arXiv:0709.3421 [hep-th]].

[10] C. Hoyos-Badajoz, C. Nunez and I. Papadimitriou, Phys. Rev. D 78, 086005 (2008) [arXiv:0807.3039 [hep-th]].

[11] A. H. Chamseddine and M. S. Volkov, Phys. Rev. Lett. 79, 3343 (1997) [arXiv:hepth/9707176]. J. M. Maldacena and C. Nunez, Phys. Rev. Lett. 86, 588 (2001) [arXiv:hepth/0008001].

[12] R. Minasian, M. Petrini and A. Zaffaroni, JHEP 1004, 080 (2010) [arXiv:0907.5147 [hepth]].

[13] J. Gaillard, D. Martelli, C. Nunez and I. Papadimitriou, Nucl. Phys. B 843, 1 (2011) [arXiv:1004.4638 [hep-th]].

[14] N. Halmagyi, arXiv:1003.2121 [hep-th].

[15] A. Dymarsky, I. R. Klebanov and N. Seiberg, JHEP 0601, 155 (2006) [arXiv:hepth/0511254].

[16] R. P. Andrews and N. Dorey, Nucl. Phys. B 751, 304 (2006) [arXiv:hep-th/0601098]. R. P. Andrews and N. Dorey, Phys. Lett. B 631, 74 (2005) [arXiv:hep-th/0505107].

[17] E. Witten, Adv. Theor. Math. Phys. 2, 505 (1998) [arXiv:hep-th/9803131].

[18] T. Sakai and S. Sugimoto, Prog. Theor. Phys. 113, 843 (2005) [arXiv:hep-th/0412141].

[19] O. Aharony, J. Sonnenschein and S. Yankielowicz, Annals Phys. 322, 1420 (2007) [arXiv:hep-th/0604161].

[20] J. Gaillard and D. Martelli, arXiv:1008.0640 [hep-th].

[21] C. Nunez, A. Paredes and A. V. Ramallo, Adv. High Energy Phys. 2010, 196714 (2010) [arXiv:1002.1088 [hep-th]].

[22] C. Nunez, I. Papadimitriou and M. Piai, Int. J. Mod. Phys. A 25, 2837 (2010) [arXiv:0812.3655 [hep-th]].

[23] S. S. Gubser, A. A. Tseytlin, and M. S. Volkov, JHEP 0109:017,2001 (JHEP 0109:017,2001).

[24] E. Caceres, R. Flauger, M. Ihl, and T. Wrase, JHEP 0803:020,2008 (Nov., 2007). 
[25] E. Caceres, R. Flauger, and T. Wrase, arXiv:0908.4483.

[26] G. T. Horowitz, A. Strominger, Nucl. Phys. B360, 197-209 (1991).

[27] M. J. Duff, J. X. Lu, Nucl. Phys. B416, 301-334 (1994). [hep-th/9306052].

[28] M. J. Duff, R. R. Khuri, J. X. Lu, Phys. Rept. 259, 213-326 (1995). [hep-th/9412184].

[29] M. Atiyah, J. M. Maldacena and C. Vafa, J. Math. Phys. 42 (2001) 3209 [arXiv:hepth/0011256]. C. Vafa, J. Math. Phys. 42, 2798 (2001) [arXiv:hep-th/0008142].

J. D. Edelstein and C. Nunez, JHEP 0104, 028 (2001) [arXiv:hep-th/0103167].

[30] A. Brandhuber, Nucl. Phys. B 629, 393 (2002) [arXiv:hep-th/0112113].

[31] J. M. Maldacena and H. S. Nastase, JHEP 0109, 024 (2001) [arXiv:hep-th/0105049].

[32] C. G. . Callan, J. A. Harvey and A. Strominger, Nucl. Phys. B 359 (1991) 611.

[33] A. Buchel, Nucl. Phys. B 600 (2001) 219 [arXiv:hep-th/0011146]. A. Buchel, C. P. Herzog, I. R. Klebanov, L. A. Pando Zayas and A. A. Tseytlin, JHEP 0104 (2001) 033 [arXiv:hepth/0102105]. S. S. Gubser, C. P. Herzog, I. R. Klebanov and A. A. Tseytlin, JHEP 0105 (2001) 028 [arXiv:hep-th/0102172]. L. A. Pando Zayas and C. A. Terrero-Escalante, JHEP 0609 (2006) 051 [arXiv:hep-th/0605170]. O. Aharony, A. Buchel and P. Kerner, Phys. Rev. D 76 (2007) 086005 [arXiv:0706.1768 [hep-th]]. M. Mahato, L. A. Pando Zayas and C. A. Terrero-Escalante, JHEP 0709 (2007) 083 [arXiv:0707.2737 [hep-th]]. A. Buchel, arXiv:1012.2404 [hep-th].

[34] G. T. Horowitz and D. L. Welch, Phys. Rev. D 49, 590 (1994) [arXiv:hep-th/9308077].

[35] M. Schvellinger, JHEP 0409, 057 (2004) [arXiv:hep-th/0407152]. P. McGuirk, G. Shiu and Y. Sumitomo, Nucl. Phys. B 842, 383 (2010) [arXiv:0910.4581 [hep-th]].

[36] I. Bena, M. Grana and N. Halmagyi, JHEP 1009, 087 (2010) [arXiv:0912.3519 [hep-th]]. I. Bena, G. Giecold and N. Halmagyi, arXiv:1011.2195 [hep-th].

[37] J. Gaillard and J. Schmude, JHEP 1002, 032 (2010) [arXiv:0908.0305 [hep-th]].

[38] F. Bigazzi, A. L. Cotrone, J. Mas, A. Paredes, A. V. Ramallo and J. Tarrio, JHEP 0911, 117 (2009) [arXiv:0909.2865 [hep-th]]. F. Bigazzi, A. L. Cotrone and J. Tarrio, JHEP 1002, 083 (2010) [arXiv:0912.3256 [hep-th]]. F. Bigazzi and A. L. Cotrone, JHEP 1008, 128 (2010) [arXiv:1006.4634 [hep-ph]]. M. Mia, K. Dasgupta, C. Gale and S. Jeon, Nucl. Phys. B 839 (2010) 187 [arXiv:0902.1540 [hep-th]]. F. Bigazzi, A. L. Cotrone, J. Mas, D. Mayerson and J. Tarrio, arXiv:1101.3560 [hep-th].

[39] F. Chen, K. Dasgupta, P. Franche, S. Katz and R. Tatar, arXiv:1007.5316 [hep-th]. 
MCTP-11-01

UTTG-01-11

\title{
Heating up the Baryonic Branch with U-duality: a unified picture of conifold black holes
}

\author{
Elena Cáceres*1 ${ }^{*}$ Carlos Núñez ${ }^{\dagger 2}$ and Leopoldo A. Pando Zayas **3 \\ * Facultad de Ciencias \\ Universidad de Colima \\ Bernal Diaz del Castillo 340, Colima, México. \\ and \\ Theory Group, Department of Physics, \\ University of Texas at Austin, Austin, TX 78727, USA. \\ $\dagger$ Department of Physics \\ University of Swansea, Singleton Park \\ Swansea SA2 8PP \\ United Kingdom. \\ ** Michigan Center for Theoretical Physics \\ Randall Laboratory of Physics, the University of Michigan \\ Ann Arbor, MI 48109-1040. USA
}

\begin{abstract}
We study different aspects of a U-duality recently presented by Maldacena and Martelli and apply it to non-extremal backgrounds. In particular, starting from new non-extremal wrapped D5 branes we generate new non-extremal generalizations of the Baryonic Branch of the Klebanov-Strassler solution. We also elaborate on different conceptual aspects of these U-dualities, like its action on (extremal and non-extremal) Dp branes, dual models for YangMills-like theories, generic asymptotics and decoupling limit of the generated solutions.
\end{abstract}

\footnotetext{
${ }^{1}$ elenac@zippy.ph.utexas.edu

${ }^{2}$ c.nunez@swansea.ac.uk

${ }^{3}$ lpandoz@umich.edu
} 


\section{Contents}

1 Introduction $\quad 2$

1.1 General Idea of this Paper . . . . . . . . . . . . . . . . . . . 3

2 A warm-up example: Rotation of Dp-branes 4

2.1 "Rotation" in p directions . . . . . . . . . . . . . . . . . 4

2.1.1 Example: rotation of a dual to a Yang-Mills-like theory . . . . . . . . 7

2.2 "Rotation" in p-q directions . . . . . . . . . . . . . . . . . . 8

3 Black Holes in $\mathcal{N}=1$ SUSY Solutions $\quad 9$

3.1 A new 'seed' solution . . . . . . . . . . . . . . . . . . . . . 11

3.2 Asymptotics . . . . . . . . . . . . . . . . . . . . . 13

3.2.1 UV expansion . . . . . . . . . . . . . . . . . . . 13

3.2 .2 Near Horizon Asymptotics . . . . . . . . . . . . . . . . . . . . . . . . . 13

3.3 Numerics ............................. . . . 14

3.3.1 Comments about the numerical method . . . . . . . . . . . 14

3.4 The rotated solution . . . . . . . . . . . . . . . . . 15

3.5 Comments on thermodynamics . . . . . . . . . . . . . . . . . . 16

4 About decoupling limits $\quad 19$

4.1 Choice of Parameters for $\mathcal{N}=1$ Black Holes . . . . . . . . . . . . . . . . . . 20

5 Conclusions and Future Directions $\quad 21$

A Appendix: U-duality in the case of flat Dp-branes 23

B Appendix: Black Dp branes $\quad 24$

C Appendix: Some details on the U-duality 25

D Appendix: Another solution generating algorithm 27

E Appendix: U-duality for the wrapped D5 branes Black Hole 29

F Appendix: The equations of motion $\quad 31$ 


\section{Introduction}

The Maldacena conjecture [1] and some of its refinements [2] are the guiding principle behind much of the progress in the interface String Theory-Quantum Field theory in the last twelve years. The influence of this approach extends to toy models with different number of supersymmetries (SUSY's), systems at finite temperature and/or finite density and lower dimensional systems. The applications to physically relevant systems do not seem to be exhausted and on the contrary, increase with time. In this sense, finding new (trustable) solutions to the equations of motion of String Theory (even in the point particle and classical approximation) has become an industry with various applications in Physics and Mathematics. Solution generating techniques have certainly played a role. Some examples worth mentioning are the combination of T-dualities and shifts of coordinates that generated solutions dual to minimally SUSY superconformal field theories (beta deformations) or duals to non-relativistic field theories $[3]^{1}$.

In this paper we will focus our attention on a particular solution generating technique that was presented in [6]. The procedure suggested by these authors starts by taking a solution in type IIB string theory proposed to be dual to (a suitably UV-completed version of) $\mathcal{N}=1$ Super Yang-Mills in four dimensions. The solution has the topology $R^{1,3} \times M_{6}$ where $M_{6}$ preserves four supercharges. The algorithm to generate the new solutions goes as follows: first apply a set of three T-dualities in the $R^{3}$ directions, which leaves us with a IIA configuration; lift this configuration to M-theory and boost-with rapidity $\beta$-in the eleventh direction; then reduce to IIA and T-dualize back in $R^{3}$. This generates a solution that roughly speaking is the dual description to the baryonic branch [7] of the Klebanov-Strassler field theory [8]. For all the technical details of this procedure the reader can refer to [6] or to our Appendix E.

The 'seed solution' (as we will refer to the initial solution on which the generating algorithm is applied) was discussed in the papers [9] (Section 8) and [10] (Section 4.3). In a particular limit, this becomes the exact solution discussed in [11].

We will also refer to the solution generating algorithm, that is a U-duality, as 'rotation' in a sense explained below and in previous papers. In the following we will emphasize some aspects of the generating technique presented in [6] that we find particularly interesting and have not been explicitly discussed in the existing bibliography:

- In the case of [6], the rotation generates D3 brane charge. This is the reason why the $S O(1,3)$ isometry of the background is untouched, in spite of doing different operations in time (boost) and in the $R^{3}$ directions (T-dualities). As a by-product of the generation of D3 branes the dilaton is invariant under this set of operations.

\footnotetext{
${ }^{1}$ In other areas of Physics, solution generating techniques play a major role. For example, many of the interesting solutions in higher dimensional gravity were constructed using solution generating algorithms. See for example [4] for original papers and [5] for a nice summary with an important application.
} 
- It can be seen that this U-duality (sequence of T-dualities, lifts and boosts) described above is equivalent to a particular case of 'rotation' in the $S U(3)$ structure of the manifold $M_{6}$ which is characterized by a complex 3 -form $\Omega_{3}$ and a 2 -form $J_{2}$ - see the papers [12] and [13]. This is another way of understanding the presence of the $S O(1,3)$ isometry: from this perspective, all the rotation 'occurs' in the internal $M_{6}$.

- The rotation generates a solution that contains two free parameters, the value of the dilaton at infinity (already present in the seed solution) and the boost parameter $\beta$. In order for the final background to be dual to the Klebanov-Strassler QFT decoupled from gravity, we must take the limit $\beta \rightarrow \infty$ so that the generated warp factor vanishes asymptotically. In this sense, the seed solution describes a field theory coupled to gravity. Only in a particular limit-see the discussion in [6], [13], we approach the near brane solution of [11].

- This rotation or U-duality, is a very curious operation from the viewpoint of the dual field theory. Indeed, it generates (aside from the bifundamental fields) new global symmetries, like the baryonic $U(1)$.

- In the same vein: the connection between the two field theories from a geometric viewpoint (Field theory at strong coupling!) is just the mentioned U-duality starting from the $D 5$-brane side. From a (weakly interacting) field theoretic viewpoint the operation is very subtle [6]. Indeed, it involves a second order fluctuation expansion (mass spectrum) in the classical and weakly coupled $\mathrm{F}$ and $\mathrm{D}$ term equations on the quiver side [15], that is matched with a classical and weakly coupled expansion of the twisted compactification of the theory in the wrapped D5 brane mass spectrum [16].

- After the first set of T-dualities described above, we have a IIA background with D2 brane charges. When lifted to M-theory we have M2 branes, that after boosted generate M2 charge and an M-wave. When reduced, this generates a D0, D2 and NS $H_{3}$ field. The generation of the $H_{3}$ in the presence of $\mathrm{RR}$ three-form implies the need to generate $F_{5}$. See Appendix E for technical details.

- We emphasized that the U-duality ( chain of T-dualities, lifts and boost) described above is a particular case of the rotation of $J_{2}, \Omega_{3}$ discussed in [12], [13], [14]. On the other hand, the rotation of $J_{2}, \Omega_{3}$ relies on the background being supersymmetric, while the U-duality can be also applied to Non-SUSY backgrounds. This will play an important role in the rest of this paper.

\subsection{General Idea of this Paper}

In this paper we will try to gain a different perspective on this solution generating technique. We will not focus on the rotation of $J, \Omega$ but rely on the U-duality description (in spite of the 
latter being a particular case of the former). Our interests will be two-fold. On one hand, we will try to get a better handle on the generating algorithm by changing it (suggesting other related algorithms) and applying it to various cases. On the other hand, we will apply it to backgrounds that do not preserve SUSY. We will study the effect of the rotation on black hole solutions, generating new non-extremal solutions with horizons and other curious features that will be discussed. We will also start the study of the properties of those newly generated solutions.

This paper is organized as follows: In sections 2 and 2.1 as a warm-up exercise we discuss a chain of dualities and boost acting on non-extremal Dp branes. In section 2.1.1 we will apply this to rotate a background dual to a version of Yang-Mills. Then, in section 2.2 we will study a variation of this U-duality that will clarify various aspects of the coming material. In Section 3 we will U-dualize a new solution describing the non-extremal deformation of a stack of $N_{c}$ D5 branes wrapping a two-cycle inside the resolved conifold. We will elaborate upon various aspects of this particular rotation in section 3.5. Finally in Section 4 we comment on decoupling aspects of the rotation. Various appendices complement our presentation. They have been written with plenty of detail hoping that colleagues working on these topics will find them useful. We close with a summary, conclusions and a list of possible future projects.

\section{A warm-up example: Rotation of Dp-branes}

In this section, we will start with a simple example of the 'rotation' procedure. That is, a sequence of T-dualities, bringing the background to a type IIA solution of the supergravity equations of motion, followed by a lift to eleven dimensions, where we will boost the configuration. We will then reduce to IIA and T-dualize back, to what we will call the 'rotated background'. As a toy example, in this section we will rotate flat $D p$ branes; first in $p$ directions and then in $p-q$ directions. We will do this in detail to appreciate the differences this introduces in the generated solution. In Appendix D, we will propose and analyze another possible U-duality to generate new solutions.

\section{1 "Rotation" in $\mathrm{p}$ directions}

Consider backgrounds of IIA/IIB of the form,

$$
\begin{aligned}
& d s^{2}=H(\rho)^{-1 / 2}\left[-h(\rho) d t^{2}+d \vec{x}_{p}^{2}\right]+H(\rho)^{1 / 2}\left[\frac{d \rho^{2}}{h(\rho)}+\rho^{2} d \Omega_{8-p}\right] \\
& e^{2 \phi[\text { initial }]}=e^{2 \phi(\infty)} H^{\frac{3-p}{2}} \\
& * F_{p+2}=\mathcal{Q} V_{o l} \Omega_{8-p}, \\
& F_{p+2}=-\partial_{\rho} A(\rho) d t \wedge d x_{1} \wedge \ldots \wedge d x_{p} \wedge d \rho
\end{aligned}
$$


with,

$$
H(\rho)=1+\left(\frac{L_{p}}{\rho}\right)^{7-p}, h(\rho)=1-\left(\frac{R_{T}}{\rho}\right)^{7-p}, \quad A(\rho)=\frac{\alpha}{g_{s} H(\rho)},
$$

where $\alpha=\frac{\tilde{Q}}{L_{p}^{7-p}}$ and $\mathcal{Q}=(7-p) \tilde{Q}$ is related to the charge of the solution (see Appendix B for details). If we choose $R_{T}=0$, the configuration above is typically singular (except for $p=3$ ) and preserves 16 SUSYs. Recall that for even (odd) values of $p$, we are dealing with a solution of Type IIA(B) supergravity.

Now, we will 'rotate' this background, by first T-dualizing in the $p$ directions, this will bring us to a IIA solution, we will lift the solution to eleven-dimensional supergravity and perform a boost of rapidity $\beta$ in the eleventh-direction. We will then reduce to IIA and T-dualize back in the $\vec{x}_{p}$ coordinates to obtain what we call the 'rotated background'. Our final rotated background is given $\mathrm{by}^{2}$,

$$
\begin{aligned}
& d s^{2}=\frac{H(\rho)^{-1 / 2}}{R(\rho)}\left[-h(\rho) d t^{2}+d \vec{x}_{p}^{2}\right]+R(\rho) H(\rho)^{1 / 2}\left[\frac{d \rho^{2}}{h(\rho)}+\rho^{2} d \Omega_{8-p}\right] \\
& e^{2 \phi[\text { final }]}=e^{2 \phi[\text { initial }]} R(\rho)^{3-p}=e^{2 \phi(\infty)}\left(H(\rho) R(\rho)^{2}\right)^{\frac{3-p}{2}} \\
& F_{p+2}=-\partial_{\rho} a(\rho) d t \wedge d x_{1} \wedge \ldots \wedge d x_{p} \wedge d \rho
\end{aligned}
$$

where we have defined,

$$
\begin{aligned}
& R^{2}=(A(\rho) \sinh \beta+\cosh \beta)^{2}-\frac{h(\rho) \sinh ^{2} \beta}{g_{s}^{2} H(\rho)^{2}} \\
& a=\frac{1}{R^{2}}\left[A(\rho) \cosh 2 \beta+\left(A(\rho)^{2}+1\right) \cosh \beta \sinh \beta-\frac{h(\rho)}{2 g_{s}^{2} H(\rho)^{2}} \sinh (2 \beta)\right]
\end{aligned}
$$

We have explicitly checked that the rotated background is a solution of the equations of motion. Notice that eq. (2.6) can be written as

$$
R^{2}=\cosh ^{2} \beta+A(\rho) \sinh 2 \beta+\left(\frac{R_{T}}{L_{p}}\right)^{7-p} \frac{\sinh ^{2} \beta}{H(\rho) g_{s}^{2}}
$$

which makes clear that $R$ is strictly positive. In a similar way, eq. (2.7) can be written as

$$
a=\frac{1}{H R^{2}}\left[\frac{1}{g_{s}} \alpha \cosh (2 \beta)+\left(\frac{R_{T}}{L_{p}}\right)^{7-p} \frac{\sinh \beta \cosh \beta}{g_{s}^{2}}+H(\rho) \sinh \beta \cosh \beta\right] \text {. }
$$

The metric (2.3) has the structure of a warped space,

$$
d s^{2}=\mathcal{H}(\rho)^{-1 / 2}\left[-h(\rho) d t^{2}+d \vec{x}_{p}^{2}\right]+\mathcal{H}(\rho)^{1 / 2}\left[\frac{d \rho^{2}}{h(\rho)}+\rho^{2} d \Omega_{8-p}\right],
$$

\footnotetext{
${ }^{2}$ In Appendix A, we will present the intermediate steps of this calculation for the extremal case $h=1$.
} 
where the new warp factor is

$$
\mathcal{H}(\rho)=H(\rho) R(\rho)^{2}=H(\rho) \cosh ^{2} \beta+\frac{\alpha}{g_{s}} \sinh 2 \beta+\left(\frac{R_{T}}{L_{p}}\right)^{7-p} \frac{\sinh ^{2} \beta}{g_{s}^{2}}
$$

Note that $\mathcal{H}(\rho)$ is a harmonic function of the transverse space. Hence, after the rotation in $p$ directions we are left with a $D p$ brane solution. Indeed, in terms of the new warp factor, the dilaton and gauge potential are

$$
\begin{aligned}
& e^{2 \phi[\text { final }]}=e^{2 \phi(\infty)} \mathcal{H}(\rho)^{\frac{3-p}{2}} \\
& \partial_{\rho} a(\rho)=\left[\frac{\alpha}{g_{s}}+\left(\frac{R_{T}}{L_{p}}\right)^{7-p} \frac{\tanh \beta}{g_{s}^{2}}\right] \partial_{\rho}\left(\frac{1}{\mathcal{H}(\rho)}\right)=\tilde{\alpha} \partial_{\rho}\left(\frac{1}{\mathcal{H}(\rho)}\right),
\end{aligned}
$$

which together with (2.10) define a $D p$ brane background with a $\beta$ dependent $\mathrm{RR}$ charge. At infinity the warp factor asymptotes to a constant,

$$
\mathcal{H}(\rho) \sim \cosh ^{2} \beta+\frac{\alpha}{g_{s}} \sinh 2 \beta+\left(\frac{R_{T}}{L_{p}}\right)^{7-p} \frac{\sinh ^{2} \beta}{g_{s}^{2}},
$$

and the space is asymptotically flat, as expected. However, it is interesting to note that even if we start with the Dp branes after the decoupling limit is taken, that is $H=\frac{L_{p}^{7-p}}{\rho^{7-p}}$, we will have in the UV that,

$$
\mathcal{H}(\rho) \sim \frac{L_{p}^{7-p}}{\rho^{7-p}} \cosh ^{2} \beta+\frac{\alpha}{g_{s}} \sinh 2 \beta+\left(\frac{R_{T}}{L_{p}}\right)^{7-p} \frac{\sinh ^{2} \beta}{g_{s}^{2}}
$$

So, again, the warp factor asymptotes to a constant. In other words, this rotation is taking the configuration out of the decoupling limit or coupling the field theory modes to gravity.

As is well known, before the rotation, the charge of the Dp brane solution is quantized. After the rotation the charge is,

$$
\begin{aligned}
\frac{1}{\left(2 \pi l_{s}\right)^{7-p}} \int_{S^{8-p}} * F_{p+2} & =\tilde{\alpha} \rho^{8-p} \mathcal{H}^{\prime}(\rho) V o l_{S^{8-p}} \\
& =\mathrm{Q} \cosh ^{2} \beta+R_{T}{ }^{7-p} \frac{c_{p}}{2 g_{s}} \sinh 2 \beta
\end{aligned}
$$

where $c_{p}=\frac{(7-p) V o l_{S} 8-p}{\left(2 \pi l_{s}\right)^{7-p} g_{s}}$. Note that, generically, the charge is not quantized. This is not unusual since the supergravity dualities involved in the rotation procedure are a symmetry of the supergravity equations and not of the full string theory.

We will now move to study a more interesting example from the viewpoint of gauge-strings duality. We will apply the rotation procedure to the a non-SUSY Yang-Mills-like theory, first presented in [17] and further studied in [18], [19]. 


\subsubsection{Example: rotation of a dual to a Yang-Mills-like theory}

The original background, consists of the decoupling limit of a stack of $N_{c}$ D4 branes wrapping a circle with SUSY breaking boundary conditions. It was discussed with details in various publication, see for example [17], [18], [19]. We summarize it here (in string frame $)^{3}$

$$
\begin{aligned}
& d s^{2}=H(\rho)^{-1 / 2}\left[-h(\rho) d t^{2}+d \vec{x}_{123}^{2}+f(\rho) d x_{4}^{2}\right]+H(\rho)^{1 / 2}\left[\frac{d \rho^{2}}{f(\rho) h(\rho)}+\rho^{2} d \Omega_{4}\right], \\
& e^{2 \phi[\text { initial }]}=g_{s}^{2} H^{-\frac{1}{2}} \\
& F_{6}=-\partial_{\rho} A(\rho) d t \wedge d x_{1} \wedge \ldots \wedge d x_{4} \wedge d \rho \quad F_{4}=* F_{6}
\end{aligned}
$$

where $A(\rho)=\frac{1}{g_{s} H(\rho)}$ and $V o l_{4}$ is the volume of the unit four- sphere $\Omega_{4}$.. The functions (in the low/zero temperature phase) are given by

$$
H=\left(\frac{L}{\rho}\right)^{3}, \quad f=1-\left(\frac{R_{k k}}{\rho}\right)^{3}, \quad h=1,
$$

where $L^{3}=N_{c} \pi g_{s} l_{s}^{3}$ and the coefficient $R_{k k}$ is a free parameter. In the high temperature phase (with the coordinate $x_{4}$ compactified) we have,

$$
H=\left(\frac{L}{\rho}\right)^{3}, \quad h=1-\left(\frac{R_{T}}{\rho}\right)^{3}, \quad f=1
$$

where $R_{T}$ is a free parameter related to the temperature of the system,

$$
T=\frac{3}{4 \pi}\left(\frac{L^{3}}{R_{T}}\right)^{-1 / 2}
$$

Proceeding as described in the previous section we rotate the background by applying the Uduality already discussed: four T-dualities in the worldvolume coordinates, uplift to M-theory, boost, reduce to IIA and T dualize back. The rotated background is,

$$
\begin{aligned}
& d s^{2}=\mathcal{H}(\rho)^{-1 / 2}\left[-h(\rho) d t^{2}+d \vec{x}_{123}^{2}+f(\rho) d x_{4}^{2}\right]+\mathcal{H}(\rho)^{1 / 2}\left[\frac{d \rho^{2}}{f(\rho) h(\rho)}+\rho^{2} d \Omega_{4}\right] \\
& e^{2 \phi[\text { final }]}=g_{s}^{2} \mathcal{H}^{-\frac{1}{2}} \\
& F_{6}=a^{\prime}(\rho) d t \wedge d x_{1} \wedge \ldots \wedge d x_{4} \wedge d \rho
\end{aligned}
$$

where the functions $\mathcal{H}(\rho), a(\rho)$ now read

$$
\begin{aligned}
& \mathcal{H}(\rho)=H(\rho) \cosh ^{2} \beta+\frac{1}{g_{s}} \sinh 2 \beta+\left(\frac{R_{T}}{L}\right)^{3} \frac{\sinh ^{2} \beta}{g_{s}^{2}} \\
& a(\rho)=\frac{1}{R(\rho)^{2}}\left[A(\rho) \cosh 2 \beta+\left(A(\rho)^{2}+1\right) \cosh \beta \sinh \beta-\frac{f(\rho) h(\rho) \sinh (2 \beta)}{2 g_{s}^{2} H(\rho)^{2}}\right]
\end{aligned}
$$

\footnotetext{
${ }^{3}$ In [18] the authors performed a rescaling of the RR potential: $C_{p+1} \rightarrow \frac{\kappa_{0}^{2} \mu_{6-p}}{\pi} C_{p+1}$ and as a result the $\mathrm{RR}$ charge in their paper is measured in units of $2 \pi$. We do not perform such rescaling here.
} 
and $R(\rho)^{2}=\frac{\mathcal{H}(\rho)}{H(\rho)}$. As before, one can check explicitly that the background above is a solution of the eqs. of motion, but for this we must set either $R_{T}=0$ for the low/zero temperature phase or set $R_{k k}=0$, compactifying the $x_{4}$ direction, in the high temperature phase.

As explained in the previous section, in spite of having started with a decoupled background, after the rotation we have an asymptotically flat space, indicating that the rotation has coupled the field theory modes to the gravitational ones.

\section{2 "Rotation" in p-q directions}

We will study now a peculiar situation, where we have Dp branes and we separate a $q$ manifold from the $p+1$ dimensional world-volume. The internal manifold need not be specified. We will impose on our U-duality that:

- after the initial T-dualities, we are in IIA background, so that this is upliftable to Mtheory

- that the boost generates a NS $H_{3}$ field, or what is equivalent, that the IIA configuration after the initial T-dualities contains an electric $F_{4}$.

We will assume (though this need not be the case) that the internal q-manifold is a torus. In that case, the solution reads (in string frame as usual) ${ }^{4}$,

$$
\begin{aligned}
& d s^{2}=H^{-1 / 2}\left[-d t^{2}+d x_{p-q}^{2}+d \sigma_{q}^{2}\right]+H^{1 / 2}\left[d \rho^{2}+\rho^{2} d \Omega_{8-p}^{2}\right] \\
& F_{p+2}=\partial_{\rho} \hat{A} d t \wedge d x_{1} \wedge \ldots \wedge d x_{p-q} \wedge d \sigma_{q} \wedge d \rho, \\
& e^{2 \phi[\text { initial }]}=e^{2 \phi(\infty)} H^{\frac{3-p}{2}} .
\end{aligned}
$$

We will proceed as follows: first we will do $\mathrm{T}$ dualities in the $x_{p-q}$ directions, this will leave us in a IIA configuration, we will lift then to M-theory and boost. We reduce to IIA and T dualize back, all the details are written in Appendix C. We end up this duality-chain with a final configuration

$$
\begin{aligned}
& d s_{I I, s t}^{2}=g_{t t} d t^{2}+\frac{d \vec{x}_{p-2}^{2}}{H^{1 / 3} B^{1 / 2}}+B^{1 / 2}\left(H^{-2 / 3} d \vec{\sigma}_{2}^{2}+H^{1 / 3}\left[d \rho^{2}+\rho^{2} d \Omega_{8-p}^{2}\right]\right), \\
& F_{p+2}=\partial_{\rho} \hat{A}\left(\cosh \beta+a_{t} \sinh \beta\right) \wedge d \rho \wedge d \sigma_{1} \wedge d \sigma_{2} \wedge d x_{1} \wedge \ldots \wedge d x_{p-2}, \\
& H_{3}=\sinh \beta \partial_{\rho} \hat{A} d \rho \wedge d \sigma_{1} \wedge d \sigma_{2}, \\
& F_{p}=\partial_{\rho}\left(a_{t}\right) d t \wedge d \rho \wedge d x_{1} \wedge \ldots \wedge d x_{p-2}, \\
& e^{2 \phi[\text { final }]}=B^{\frac{5-p}{2}} H^{\frac{2-p}{3}}
\end{aligned}
$$

\footnotetext{
${ }^{4}$ we are dealing here with the Lorentz invariant case here. Working with a non-extremal factor is straightforward.
} 
where we have defined (See Appendix C for a detailed derivation),

$$
\begin{aligned}
& A=H^{-2 / 3}\left[H \sinh ^{2} \beta-\cosh ^{2} \beta\right], \quad B=H^{-2 / 3}\left[H \cosh ^{2} \beta-\sinh ^{2} \beta\right], \\
& C=2 H^{-2 / 3} \sinh \beta \cosh \beta(1-H), \quad g_{t t}=\frac{4 A B-C^{2}}{4 \sqrt{B}}, \\
& a_{t}=\frac{C}{2 B}=\frac{\sinh \beta \cosh \beta(1-H)}{H \cosh ^{2} \beta-\sinh ^{2} \beta},
\end{aligned}
$$

Various aspects are worth emphasizing in this final background. Notice that:

- the metric is dual to a field theory with $S O(1, p-2)$ isometry, since

$$
\left(4 A B-C^{2}\right) H^{1 / 3}=-4 \rightarrow g_{t t}=-g_{x_{i} x_{i}}
$$

- for $p=5$ the dilaton is invariant under the whole U-duality procedure, since

$$
e^{2 \phi[\text { initial }]}=H^{\frac{3-p}{2}}=e^{2 \phi[\text { final }]}=B^{\frac{5-p}{2}} H^{\frac{2-p}{3}}
$$

- for $p=5$ the procedure is generating charge of D3 brane (represented by the $F_{p=5}$ ) where the D3 branes are extended in the $p-q$ directions. This is the reason why the dilaton does not change as the D3 branes do not couple to it. The field $H_{3}$ is also generated.

In following sections, we will study a particular case of this U-duality, for a situation in which a set of D5 branes wrap a curved two-manifold (but we also have a black hole in the metric, breaking the $S O(1,3)$ isometry), the results are qualitatively the same. One should emphasize that there is yet another way of generating NS three-form fields, that is basically starting from NS five branes in IIA wrapping a three-cycle, see [20] for details.

Also, notice that we have imposed that after the first set of T-dualities, the background is solution of IIA Supergravity (the conditions for this to happen are discussed in Appendix C). Were this not the case, we present in Appendix D another possible solution generating technique with some applications.

Now, we will move to study an interesting application of the U-dualities discussed above.

\section{Black Holes in $\mathcal{N}=1$ SUSY Solutions}

As anticipated, in this section we will apply the chain of dualities, lift and boost proposed in [6], to generate a new solution starting from a non-extremal solution in Type IIB. The interest of the original ('seed') solution is that it was argued to be dual to a field theory with minimal SUSY in four dimensions. The field theory was studied (at weak coupling) in [16] and is basically $\mathcal{N}=1$ Super-Yang-Mills plus a sets of (massive) KK vector and chiral superfields 
that UV complete the dynamics. This system was well studied and various string duals are known (particular solutions describing the field theory at strong coupling, with VEV's and certain operators deforming the Lagrangian). Let us briefly describe the general form of the string dual. The 'seed' background describes the backreaction of a set of $N_{c}$ D5 branes wrapping a two-cycle inside the resolved conifold. It consists of a metric, dilaton $\phi(\rho)$ and RR three-form $F_{3}$ and, in string frame, it reads (the coordinates used are $[t, \vec{x}, \rho, \theta, \varphi, \tilde{\theta}, \tilde{\varphi}, \psi]$ ),

$$
\begin{aligned}
d s^{2}= & e^{\phi(\rho)}\left[d x_{1,3}^{2}+e^{2 k(\rho)} d \rho^{2}+e^{2 h(\rho)}\left(d \theta^{2}+\sin ^{2} \theta d \varphi^{2}\right)+\right. \\
+ & \left.\frac{e^{2 g(\rho)}}{4}\left(\left(\tilde{\omega}_{1}+a(\rho) d \theta\right)^{2}+\left(\tilde{\omega}_{2}-a(\rho) \sin \theta d \varphi\right)^{2}\right)+\frac{e^{2 k(\rho)}}{4}\left(\tilde{\omega}_{3}+\cos \theta d \varphi\right)^{2}\right], \\
F_{(3)}= & \frac{N_{c}}{4}\left[-\left(\tilde{\omega}_{1}+b(\rho) d \theta\right) \wedge\left(\tilde{\omega}_{2}-b(\rho) \sin \theta d \varphi\right) \wedge\left(\tilde{\omega}_{3}+\cos \theta d \varphi\right)+\right. \\
& \left.b^{\prime} d \rho \wedge\left(-d \theta \wedge \tilde{\omega}_{1}+\sin \theta d \varphi \wedge \tilde{\omega}_{2}\right)+\left(1-b(\rho)^{2}\right) \sin \theta d \theta \wedge d \varphi \wedge \tilde{\omega}_{3}\right] .
\end{aligned}
$$

where $\tilde{\omega}_{i}$ are the left-invariant forms of $S U(2)$

$$
\begin{aligned}
& \tilde{\omega}_{1}=\cos \psi d \tilde{\theta}+\sin \psi \sin \tilde{\theta} d \tilde{\varphi}, \quad \tilde{\omega}_{2}=-\sin \psi d \tilde{\theta}+\cos \psi \sin \tilde{\theta} d \tilde{\varphi}, \\
& \tilde{\omega}_{3}=d \psi+\cos \tilde{\theta} d \tilde{\varphi} .
\end{aligned}
$$

This supersymmetric system was carefully studied in a series of papers [9], [10]; where it was shown that there is a combination of background functions (basically a 'change of basis') that move from a set of coupled BPS equations to a decoupled one that one can solve-up to one function, that we will call $P(\rho)$. We will not insist much with this formalism here and refer the interested reader to the original work [9], $[10]^{5}$.

We will be more restrictive and for the purposes of this paper, we will study solutions where the functions $a(\rho)=b(\rho)=0$ in the background of eq.(3.28). This is just in order to make our numerics simpler and illustrate the points we want to make. If we wanted to construct a black hole solution showing explicitly the transition between R-symmetry breaking and its restoration, we should then work without this restriction. Since in all known solutions the asymptotics of the functions $a(\rho) \sim b(\rho) \sim e^{-2 \rho}$ our asymptotic results will be qualitatively correct, but near the black hole horizon there could be important differences.

We will then proceed as follows: first we will propose a background including a black hole with the restrictions mentioned above $(a(\rho)=b(\rho)=0)$. We will then pass it through the solution generating machine. This will produce a new background, now including also $F_{5}, H_{3}$, that we will write explicitly (we have checked that the equations of motion before and after the U-duality are the same). We will be explicit about the asymptotics of each of the functions

\footnotetext{
${ }^{5}$ All this formalism was also applied to the case in which one also adds fundamental matter to the dual QFT or sources to the background above. We refer the interested reader to the review [21].
} 
near the UV and near the horizon. This will produce for us a non-extremal generalization of the type IIB string background dual to the 'baryonic branch of KS-system' -certainly with the restriction that our background does not contain the information of the breaking of the R-symmetry from $Z_{2 N} \rightarrow Z_{2}$.

\subsection{A new 'seed' solution}

In this section we consider the background presented in equation (3.28) with the restrictions $a=b=0$ but including the non-extremality factors. In string frame we have,

$$
\begin{aligned}
& d s_{I I B, s}^{2}=e^{\phi}\left[-h(\rho) d t^{2}+d x_{1}^{2}+d x_{2}^{2}+d x_{3}^{2}\right]+d s_{6, s}^{2}, \\
& d s_{6}^{2}=e^{\phi}\left[\frac{e^{2 k}}{s(\rho)} d \rho^{2}+\frac{e^{2 k}}{4}\left(\tilde{\omega}_{3}+\cos \theta d \varphi\right)^{2}+e^{2 q}\left(d \theta^{2}+\sin ^{2} \theta d \varphi^{2}\right)+\frac{e^{2 g}}{4}\left(d \tilde{\theta}^{2}+\sin ^{2} \tilde{\theta} d \tilde{\varphi}^{2}\right)\right] \\
& F_{(3)}=\frac{N_{c}}{4}\left[-\tilde{\omega}_{1} \wedge \tilde{\omega}_{2}+\sin \theta d \theta \wedge d \varphi\right] \wedge\left(\tilde{\omega}_{3}+\cos \theta d \varphi\right)=\frac{\alpha^{\prime} N_{c}}{4} w_{3},
\end{aligned}
$$

where $h(\rho), s(\rho)$ are the non-extremality functions. In the following, we will set $s(\rho)=h(\rho)$ which is simply a choice of parametrization; this implies that (on a particular solution) other background functions $\{q(\rho), g(\rho), k(\rho), \phi(\rho)\}$ need not take the same values as in the SUSY background.

The above Ansatz might be familiar to some readers. It is worth highlighting a key difference with previous work. Since our goal is to 'rotate' this solution we look for solutions with stabilized dilaton. Namely, the typical asymptotic value of the dilaton inherited from the D5 (or NS5 brane) solution is linear [32], more precisely [23] [25],

$$
e^{\phi} \sim e^{\rho} \rho^{-1 / 4}
$$

We are looking for a dilaton that stabilizes at infinity, that is, which asymptotically behaves as:

$$
e^{\phi} \sim 1+\mathcal{O}\left(e^{-8 / 3 \rho}\right)
$$

Our solution is qualitatively characterized by two parameters: one describing the nonextremality of the solution and the other the speed at which the dilaton gets stabilized.

After applying the solution generating technique, we end up with a new Type IIB background containing $F_{5}, H_{3}$ aside from the fields originally present in eq.(3.30) (all details are 
written in the Appendix E),

$$
\begin{aligned}
& d s_{I I B, s t}^{2}=H^{-1 / 2}\left[-h d t^{2}+d x_{1}^{2}+d x_{2}^{2}+d x_{3}^{2}\right]+e^{2 \phi} H^{1 / 2}\left[e^{2 k} \frac{d \rho^{2}}{h(\rho)}+\frac{e^{2 k}}{4}\left(\tilde{\omega}_{3}+\cos \theta d \varphi\right)^{2}+\right. \\
& \left.+e^{2 q}\left(d \theta^{2}+\sin ^{2} \theta d \varphi^{2}\right)+\frac{e^{2 g}}{4}\left(d \tilde{\theta}^{2}+\sin ^{2} \tilde{\theta} d \tilde{\varphi}^{2}\right)\right] \\
& H^{1 / 2}=\cosh \beta \sqrt{e^{-2 \phi}-h(\rho) \tanh ^{2} \beta}, \quad \phi_{n e w}=\phi(\rho), \\
& F_{3}=\left(\frac{\alpha^{\prime} N_{c}}{4}\right) \cosh \beta w_{3}, \quad H_{3}=-\sqrt{h}\left(\frac{\alpha^{\prime} N_{c}}{4}\right) \sinh \beta e^{2 \phi} *_{6} w_{3} \\
& F_{5}=-\tanh \beta\left(1+*_{10}\right) \operatorname{vol}_{4} \wedge d\left(\frac{h}{H}\right) .
\end{aligned}
$$

In the following, we will focus in the limit $\beta \rightarrow \infty$, this is the field theory-limit where the warp factors vanish at infinity. We will then rescale

$$
\tilde{N}=N \cosh \beta, \quad x_{i} \rightarrow \sqrt{\cosh \beta} \sqrt{\tilde{N} \alpha^{\prime}} x .
$$

With all the above the $\beta \rightarrow \infty$ limits are finite and the solution is given by

$$
\begin{aligned}
& d s_{I I B, s t}^{2}=\tilde{N} \alpha^{\prime}\left[\tilde{H}^{-1 / 2}\left(-h d t^{2}+d x_{i} d x^{i}\right)+e^{2 \phi} \tilde{H}^{1 / 2} d s_{6}^{2}\right], \\
& F_{3}=\frac{\alpha^{\prime} \tilde{N}}{4} w_{3}, \quad H_{3}=-\sqrt{h} \frac{\alpha^{\prime} \tilde{N}}{4} e^{2 \phi} *_{6} w_{3} \\
& F_{5}=-\left(\alpha^{\prime} \tilde{N}\right)^{2}\left(1+*_{10}\right) \operatorname{vol}_{4} \wedge d\left(\frac{h}{\tilde{H}}\right)
\end{aligned}
$$

with

$$
\tilde{H}^{1 / 2}=\sqrt{e^{-2 \phi}-h(\rho)}
$$

It is worth making a few observations:

- We have two new classes of solutions. The equations of motion before and after the 'rotation' are the same, thus a solution of the system of equations (3.30) furnishes a solution of the form (3.33)-(3.35).

- The non-extremality factor have made its way into the RR five form and the warp factor in eq.(3.36), but the dilaton and the three forms are unchanged from the extremal case. Notice that the factor of $\sqrt{h}$ in the NS field $H_{3}$ is canceled by the self dual in six dimensions $*_{6} w_{3}$. See Appendix E for full details.

- The black hole in the seed solution will likely have negative specific heat but its dilaton is stabilized which is a crucial difference with solutions considered previously. We will discuss what is the behavior of the solution after the rotation.

In the following section we will explicitly describe the asymptotic behavior of the solution as well as its numerical presentation in the background given by eq.(3.30). 


\section{$3.2 \quad$ Asymptotics}

We will proceed to study the asymptotics of the equations of motion and solve them numerically to obtain the new non-extremal seed solution (3.30).

\subsubsection{UV expansion}

A large $\rho$ expansion of the functions $(k, q, g, h, \phi)$, that solve the equations of motion up to terms decaying faster than $e^{-4 \rho}$ is ${ }^{6}$

$$
\begin{aligned}
& h(\rho)=e^{-8 x(\rho)} \sim 1+C_{2} e^{-\frac{8}{3} \rho} \\
& e^{2 q(\rho)} \sim \frac{N_{c}}{4}(2 \rho-1)+\frac{c}{4} e^{\frac{4}{3} \rho}, \quad e^{2 g(\rho)} \sim \frac{N_{c}}{4}(1-2 \rho)+\frac{c}{4} e^{\frac{4}{3} \rho} \\
& e^{2 k(\rho)} \sim \frac{2 c}{3} e^{\frac{4}{3} \rho}-\frac{N_{c}^{2}}{6 c}\left(25-40 \rho+16 \rho^{2}\right) e^{-\frac{4}{3} \rho}, \quad e^{4 \phi-4 \phi_{0}} \sim 1+\frac{3 N_{c}^{2}}{4 c^{2}}(1-8 \rho) e^{-\frac{8}{3} \rho}
\end{aligned}
$$

There are two parameters in the expansion above. The parameter $C_{2}$ characterizes the nonextremal behavior. The parameter $c$ plays a quite important role. It is one of the integration constants of the BPS eqs. As an expansion in inverse powers of this constant it is possible to write a solution for the background in eq.(3.28)-see for example [10], [22]-such that when passed through this solution generating technique, it results in the Baryonic Branch dual solution of [7]. Physically (and in the background before the rotation), the constant $c$ is describing the coupling of the dynamics on the five-brane to gravity and ultimately to the full string theory, it takes the configuration out of its 'near brane' limit and can be thought of as indicating the insertion of an irrelevant operator in the QFT lagrangian.

\subsubsection{Near Horizon Asymptotics}

Near the horizon, $\rho=\rho_{h}$, we expand the equations of motion in power series up to order 7 . We have found,

$$
\begin{aligned}
e^{-8 x(\rho)} & =x_{1}\left(\rho-\rho_{h}\right)+x_{2}\left(\rho-\rho_{h}\right)^{2}+x_{3}\left(\rho-\rho_{h}\right)^{3}+\cdots+x_{8}\left(\rho-\rho_{h}\right)^{8} \\
e^{2 q(\rho)} & =q_{0}+q_{1}\left(\rho-\rho_{h}\right)+q_{2}\left(\rho-\rho_{h}\right)^{2}+\cdots+q_{7}\left(\rho-\rho_{h}\right)^{7} \\
e^{2 g(\rho)} & =g_{0}+h_{1}\left(\rho-\rho_{h}\right)+g_{2}\left(\rho-\rho_{h}\right)^{2}+\cdots+g_{7}\left(\rho-\rho_{h}\right)^{7} \\
e^{2 k(\rho)} & =k_{0}+k_{1}\left(\rho-\rho_{h}\right)+k_{2}\left(\rho-\rho_{h}\right)^{2}+\cdots+k_{7}\left(\rho-\rho_{h}\right)^{7} \\
e^{4 \Phi(\rho)} & =f_{0}+f_{1}\left(\rho-\rho_{h}\right)+f_{2}\left(\rho-\rho_{h}\right)^{2}+\cdots+f_{7}\left(\rho-\rho_{h}\right)^{7}
\end{aligned}
$$

Demanding that the expansions in eq.(3.38) satisfy the equations of motion of the system we determine $x_{2}, \ldots, h_{1} \ldots, g_{1} \ldots, k_{1} \ldots, f_{1} \ldots$ in terms of $x_{1}, h_{0}, g_{0}, k_{0}, f_{0}$ and thus, there are only 5

\footnotetext{
${ }^{6}$ Note that the UV expansion for $h(\rho)=e^{-8 x}$ could have started with a lower order term such as $e^{-2 \rho}$, see Section 4.1 for a discussion on the choice of parameters.
} 



Figure 1: Solution before the rotation for Figure 2: Solution before the rotation for $c=1.97, C_{2}=-7000$. The blue, dashed, $c=125.62, C_{2}=-89$. The blue, dashed, green and orange lines represent $e^{q}, e^{k}, e^{g}$ and green ad orange lines represent $e^{q}, e^{k}, e^{g}$ and $h^{-1}$ respectively. $\quad h^{-1}$ respectively.

independent parameters coming from the expansion at the horizon. Furthermore, $x_{1}$ is related to the non-extremality parameter $\alpha$-see Appendix F for the definitions,

$$
\alpha=-\frac{x_{1} \sqrt{f_{0}} g_{0} h_{0}}{8}
$$

\subsection{Numerics}

The strategy we will follow is to numerically integrate back from infinity the equations of motion in Appendix F, using as boundary conditions the expansion in the UV-eq.(3.37)-and require that at the horizon this numerical solution matches the expansion (3.38) and its first derivatives -up to seventh order. Doing this for a given set of values $\left(c, C_{2}\right)$, determines the free parameters $\left(x_{1}, h_{0}, g_{0}, k_{0}, f_{0}\right)$. Note that not for every pair of $\left(c, C_{2}\right)$ there is a black hole solution. For some values of $\left(c, C_{2}\right)$ there will not be a horizon and for others there might be naked singularities outside the horizon. The study of the this two-parameter family of solutions for the full $\left(c, C_{2}\right)$ parameter space is an interesting question that we will not address here. We concentrate on finding particular values of $\left(c, C_{2}\right)$ that will produce black hole solutions. Needless to say, finding a solution before the rotation guarantees that we will have a solution after the rotation. Examples of numerical solutions are shown in Figure 1 and 2 and the respective dilatons in Figures 3 and 4 .

\subsubsection{Comments about the numerical method}

As mentioned above we integrate back from infinity and match with the expansion at the horizon. In order to do so we define a "mismatch" function evaluated at a point $\rho_{0}$ close to 

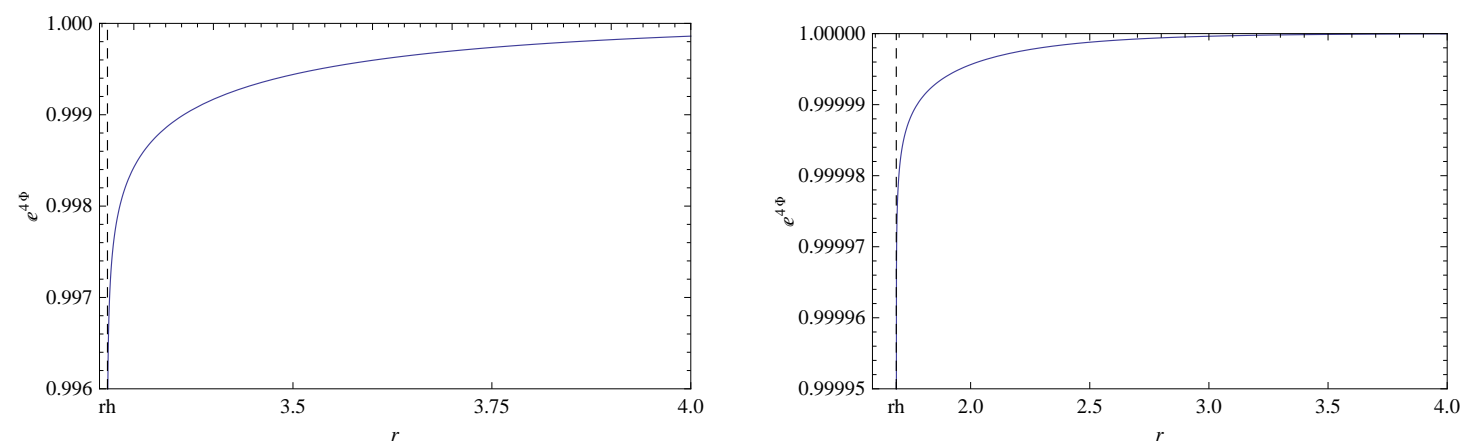

Figure 3: Dilaton, $e^{4 \Phi}$ for $c=1.97, C_{2}=$ Figure 4: Dilaton, $e^{4 \Phi}$ for $c=125.62, C_{2}=$ -7000 . -89 .

the horizon, $\rho_{0}=\rho_{h}+0.05$

$$
\begin{aligned}
m\left(\rho_{0}\right)= & {\left[g_{\text {sh }}\left(\rho_{0}\right)-g_{\text {num }}\left(\rho_{0}\right)\right]^{2}+\left[g_{\text {sh }}^{\prime}\left(\rho_{0}\right)-g_{\text {num }}^{\prime}\left(\rho_{0}\right)\right]^{2}+\left[h_{\text {sh }}\left(\rho_{0}\right)-h_{\text {num }}\left(\rho_{0}\right)\right]^{2}+} \\
& {\left[h_{\text {sh }}^{\prime}\left(\rho_{0}\right)-h_{\text {num }}^{\prime}\left(\rho_{0}\right)\right]^{2}+\left[k_{s h}\left(\rho_{0}\right)-k_{\text {num }}\left(\rho_{0}\right)\right]^{2}+\left[k_{\text {sh }}^{\prime}\left(\rho_{0}\right)-k_{\text {num }}^{\prime}\left(\rho_{0}\right)\right]^{2}+} \\
& {\left[\Phi_{\text {sh }}\left(\rho_{0}\right)-\Phi_{\text {num }}\left(\rho_{0}\right)\right]^{2}+\left[\Phi_{\text {sh }}^{\prime}\left(\rho_{0}\right)-\Phi_{\text {num }}^{\prime}\left(\rho_{0}\right)\right]^{2} . }
\end{aligned}
$$

The subscript num denotes the numerical solution obtained by integrating back from infinity and $s h$ denotes the series expansion (3.38) -evaluated at $\rho_{0}$. Recall that the expansion at the horizon depends on five free parameters, $\left(x_{1}, h_{0}, g_{0}, k_{0}, f_{0}\right)$. For a given set of $\left(c, C_{2}\right)$ we minimize the mismatch function to determine $\left(x_{1}, h_{0}, g_{0}, k_{0}, f_{0}\right)$. The parameters at infinity $\left(c, C_{2}\right)$ are adjusted so that $m\left(\rho_{0}\right)<5 \times 10^{-5}$. All the numerical procedures where done in Mathematica using WorkingPrecission 40. We also checked that the constraint $T+U=0$ (see appendix F) remains numerically negligible throughout the domain.

\subsection{The rotated solution}

Once we have obtained a numerical solution for the non-extremal background (3.30) we can easily generate the rotated solution as summarized in eq.(3.35) and outlined in the Appendix E. Before presenting the numerical results let us look at the large $\rho$ asymptotics. Using the UV expansions of the seed solution (3.37), one can obtain the black hole metric (3.35) asymptotics 
after the rotation,

$$
\begin{aligned}
-g_{t t} & \sim \frac{e^{4 / 3 \rho}}{A(\rho)}-\frac{e^{-4 / 3 \rho}}{512 c^{4} \sqrt{2} A(\rho)^{3}}\left[256 c^{4} C_{2}^{2}+27(1-8 \rho)^{2}+96 c^{2} C_{2}(-1+8 \rho)\right]+\cdots, \\
g_{x x} & \sim \frac{e^{4 / 3 \rho}}{A(\rho)}-\frac{e^{-4 / 3 \rho}}{512 c^{4} \sqrt{2} A(\rho)^{3}}\left[27(1-8 \rho)^{2}\right]+\cdots, \\
g_{\rho \rho} & \sim \frac{2 c}{3} A(\rho)+\cdots, \quad g_{\theta \theta} \sim \frac{c}{4} A(\rho)+\frac{e^{-4 / 3 \rho}}{4} A(\rho)(2 \rho-1)+\cdots, \\
g_{\psi \psi} & \sim \frac{c}{6} A(\rho)+\cdots, \quad g_{\tilde{\theta} \tilde{\theta}} \sim \frac{c}{4} A(\rho)-\frac{e^{-4 / 3 \rho}}{4} A(\rho)(2 \rho-1)+\cdots .
\end{aligned}
$$

Where we have defined the quantity

$$
c \sqrt{8} A(\rho) \equiv \sqrt{24 \rho-8 C_{2} c^{2}-3} .
$$

Using a variable $u=e^{2 \rho / 3}$ the metric can be written -to leading order- as,

$$
d s^{2}=\frac{u^{2}}{A(u)}\left(d x_{i} d x^{i}\right)+\frac{3 c A(u)}{2}\left(\frac{d u^{2}}{u^{2}}+d s_{T^{11}}^{2}\right)+\cdots \mathcal{O}\left(u^{-2}\right) .
$$

Thus, the non-extremal solution found after the rotation has the asymptotics of the KlebanovStrassler background [8]. We point out that the reason we get precisely Klebanov-Tseytlin type of asymptotics lies in our precise arrangements of coefficients in the seed solution. Namely we arranged for a seed solution with no linear dilaton and with a dilaton behavior that, in the field theory limit, eliminates the leading term in the harmonic function given in equation $(3.36)$.

With our careful choice of asymptotic conditions for the seed solution of D5 branes we have managed to construct black holes in asymptotically KT backgrounds. Our solution is different from those obtained in the traditional approach to black holes in asymptotically KT backgrounds (see, for example, [33]). One glaring difference is the form of the RR fluxes. In particular the presence of the non-extremality parameter in $F_{5}$ and in the warp factor $H(\rho)$ is completely novel.

In Figures 5-8 we present the numerical plots of the diffent metric elements after the rotation for two sets of parameters. In Figures 9 and 10 we see how the angular part of the solution approaches the $T^{1,1}$ metric for the two representative solutions.

\subsection{Comments on thermodynamics}

Let us clarify the expectations of the form of the background that arise from field theory considerations. In the previous section we obtained a solution largely characterized by two parameters $\left(c, C_{2}\right)$. After the rotation, the interpretation of $c$ has been spelled out explicitly 

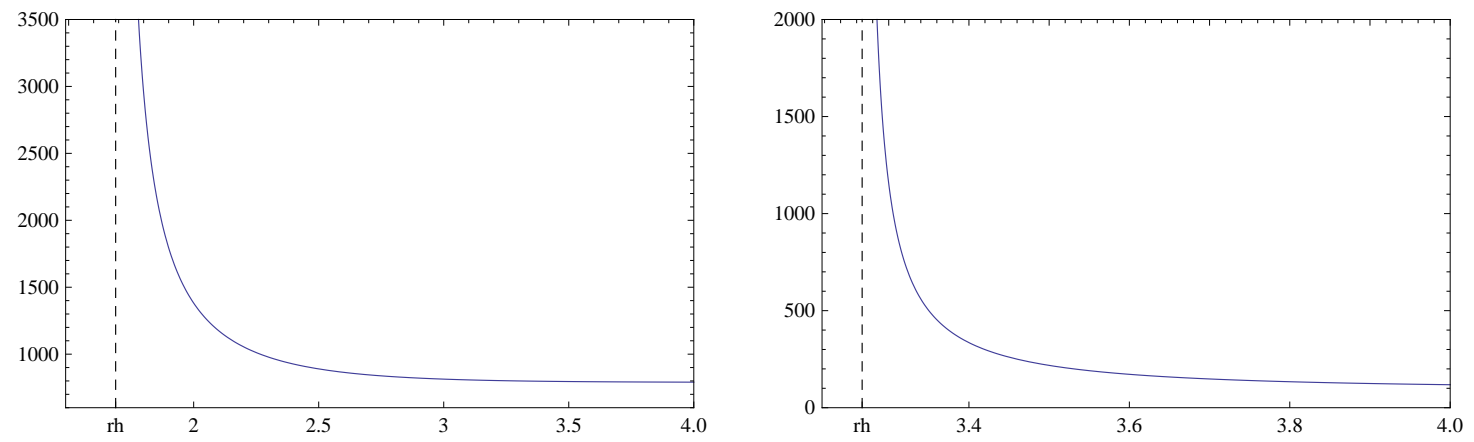

Figure 5: The $g_{r r}$ metric element for a so- Figure 6: The $g_{r r}$ metric element for a solulution after the rotation, $c=125.62, C 2=$ tion after the rotation, $c=1.97, C_{2}=-7000$. -89 .
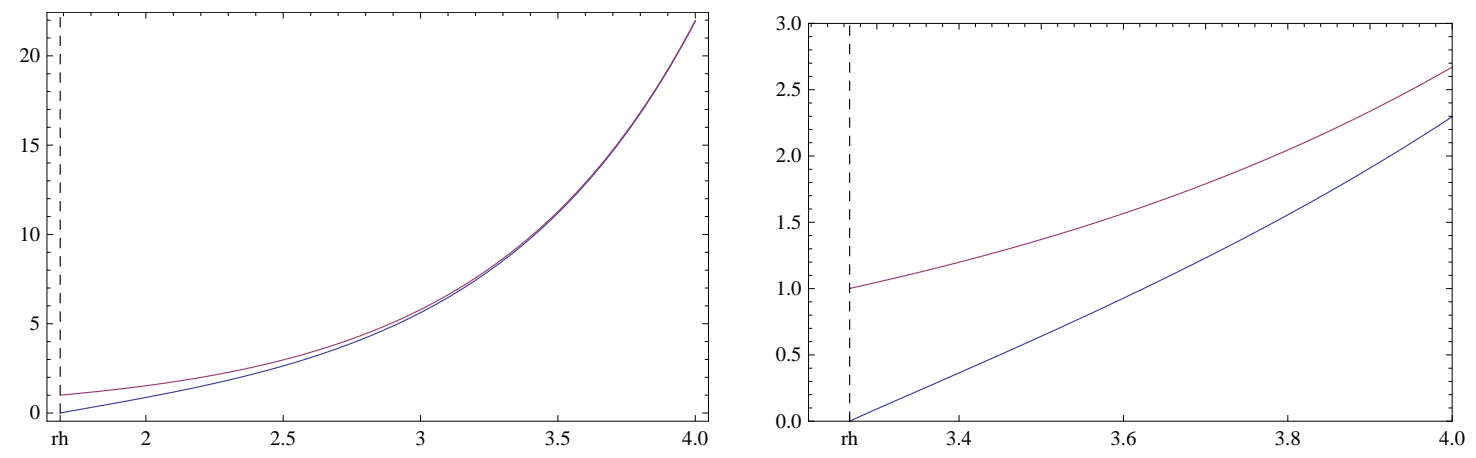

Figure 7: $g_{x x}$ and $g_{t t}$ for a solution after the Figure 8: $g_{x x}$ and $g_{t t}$ for a solution after the rotation, $c=125.62, C_{2}=-89$. rotation, $c=1.97, C_{2}=-7000$.
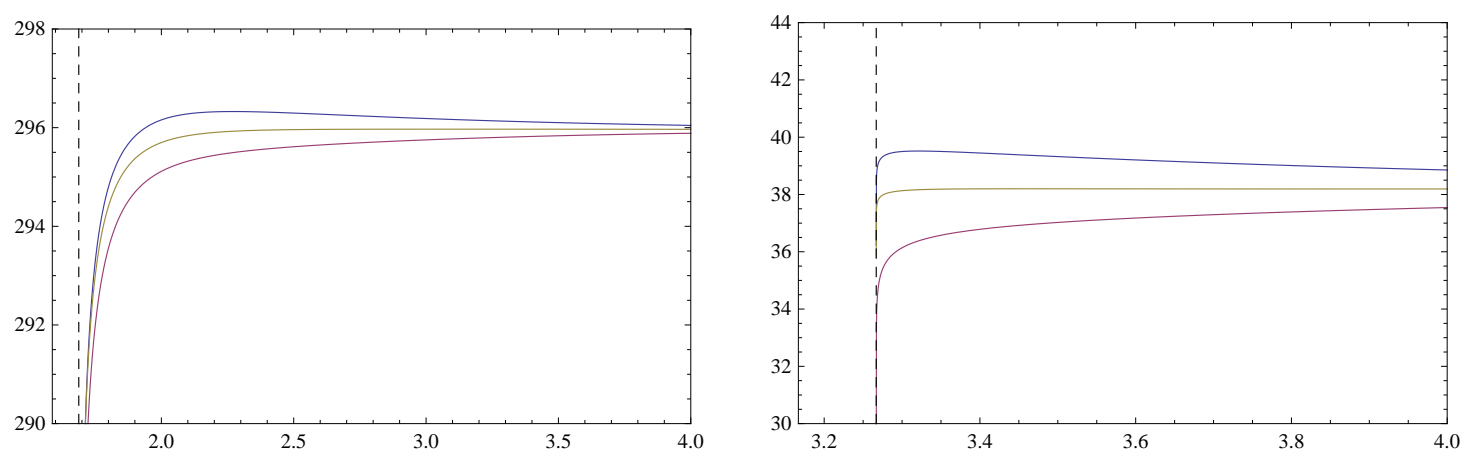

Figure 9: Angular part of the metric after Figure 10: Angular part of the metric after the rotation. We plot $g_{\theta \theta}, g_{\tilde{\theta} \tilde{\theta}}$ and $\frac{3}{2} g_{\psi \psi}$. So- the rotation. We plot $g_{\theta \theta}, g_{\tilde{\theta} \tilde{\theta}}$ and $\frac{3}{2} g_{\psi \psi}$. Solution with $c=125, C_{2}=-89$. lution with $c=1.97, C_{2}=-7000$. 
in [15], [6] and [13]. It is related to the vev of the baryon operator. The other parameter present in the solution is $C_{2}$, its interpretation coincides roughly with the temperature of the solution in the field theory.

We will present and exhaustive analysis of these classes of solutions elsewhere and will limit our attention here to a few main points. The main deterrent in presenting a final description of the thermodynamics is the need to explore a large parameter space of the solutions as well as the difficulty in matching data at asymptotic infinity with data near the horizon. The analysis is numerically costly but we emphasize that these are not difficulties of principle. Our goal in this work is to explicitly show, through numerical analysis, the existence of the conjectured solutions.

Let us show that the thermodynamic analysis can, in principle, be done explicitly for the solutions we have. For the temperature, we use the standard approach of looking at the Euclidean section and imposing the absence of conical singularities to determine the temperature of the supergravity backgrounds. Basically, for a metric with Euclidean section of the form

$$
d s^{2}=f d \tau^{2}+\frac{d \rho^{2}}{g}+\ldots,
$$

we find a temperature equal to

$$
T=\frac{1}{4 \pi} \sqrt{f^{\prime} g^{\prime}},
$$

where prime represents derivative with respect to the radial coordinate and the above expression is evaluated at the horizon. Interestingly, the temperature in both backgrounds, that is, before and after the rotation, is the same. In terms of the near horizon data we have

$$
T=\frac{1}{4 \pi} \frac{x_{1}}{\sqrt{k_{0}}} .
$$

It is worth noting that this is the temperature at the horizon. In the case of asymptotically flat backgrounds (before the rotation) we have the option of computing the temperature as seen by an observer at infinity. We refer the reader to [23] for a detailed discussion of this procedure in the D5 or NS5 background. The entropy is computed, as usual, as the area of the horizon. The most complicated quantity to compute is the free energy which is required to compute, for example, the specific heat. For the free energy we need to evaluate the action. In the case of asymptotically flat solutions for D5/NS5 branes we can essentially follow [23]. A very preliminary analysis seems to confirm our intuition about the specific heat of such solutions. Namely, evaluation of the action suggests that before the rotation the solutions obtained have negative specific heat. A precise evaluation of the action after the rotation is more subtle as naive evaluation yields divergent terms. It is likely that a more accurate approach requires some sort of holographic renormalization or substraction along the lines of [33]. Preliminary evaluation suggests a positive specific heat for the rotated solutions ${ }^{7}$.

\footnotetext{
${ }^{7}$ Dario Martelli suggested that along the lines of the first two papers in [3], some thermodynamical quantities should be invariant under T-dualities, shifts of coordinates and boosts. See also [34].
} 


\section{About decoupling limits}

It is worth discussing a bit about decoupling limits. Let us do this in the extremal case, the extension to the cases with non extremal factors follows from what we write here. The first point to emphasize is that when gravity modes are coupled to a generic D-brane configuration, the warp factors asymptote to a constant. An example of this is what happens to the flat Dp branes, with $H=1+\left(\frac{Q}{\rho}\right)^{7-p}$.

We will compare warp factors in the examples studied in the previous sections. It is enough to focus on the expressions of the metric component $g_{t t}$. In all the examples studied we found

$$
g_{t t}=\frac{4 A B-C^{2}}{4 \sqrt{B}}
$$

Indeed, in the case in which we rotate a flat Dp-brane, we have eq.(A.6) with $A, B, C$ given in eq.(A.5). In the case of the rotation in $p-q$ directions the results for $A, B, C$ are written in eq. (2.25) and in the case of the wrapped D5's are given in eq.(E.9). Again, for the point we wish to emphasize, we will focus on the extremal cases, when the rotation preserves $S O(1, p)$. So, in these cases we have ${ }^{8}$,

$$
\begin{aligned}
& g_{t t,(p-q)}=-\frac{1}{\cosh \beta \sqrt{H-\tanh ^{2} \beta}}, g_{t t, p}=-\frac{\sqrt{H}}{\sqrt{H^{2}(\hat{A} \tanh \beta-1)^{2}-\tanh ^{2} \beta}}, \\
& g_{t t, D 5}=-\frac{1}{\cosh \beta \sqrt{e^{-2 \phi}-\tanh ^{2} \beta}}
\end{aligned}
$$

The case of the rotation in $p$ directions shows that the induced charge of D0 brane (after the first set of T-dualities) as shown by eq.(A.2), enters in the new warp factor via the one-form potential $A_{1}=\hat{A} d t$. This makes the decoupling of the final configuration more unclear and typically, if we start with 'decoupled' Dp branes (that is $H \sim \rho^{p-7}$ ) we will generate a nondecoupled configuration, with an asymptotically constant warp factor as discussed around eq.(2.13). Of course, we can try to take the decoupling limit in the final configuration.

On the other hand the cases of the wrapped D5's and the rotation in $p-q$ directions (that share the fact that a D0 charge is induced by the boost only) are such that we must start with a non-decoupled configuration in order to avoid a generated background with a singularity or end of the space in the UV (zero warp factor at some finite value of the radial coordinate). Indeed, if $H=1+\left(\frac{Q}{\rho}\right)^{7-p}$ we choose a particular value of the boost parameter $\beta \rightarrow \infty$, together with a particular choice of the value of $e^{\phi(\infty)}=1$ in the D5's case) to obtain a background decoupled from gravity ${ }^{9}$.

\footnotetext{
${ }^{8}$ Gregory Giecold correctly pointed out that in the non-extremal cases the non-extremality factor $h(\rho)$ enters as expected, for example $g_{t t, D 5}=-\frac{h}{\cosh \beta \sqrt{e^{-2 \phi}-h \tanh ^{2} \beta}}$. We thank him for this and various other correct comments.

${ }^{9}$ One of the interesting observations in [6], is that in the case of the D5's the solution described in the
} 


\subsection{Choice of Parameters for $\mathcal{N}=1$ Black Holes}

Let us discuss in more detail the choice of parameters in the solution. This is a particularly sensitive question from the numerical and from the physical points of view. We note that $a$ priori there are ten parameters in our solution as corresponds to a system of five second order ordinary differential equations. The constraint lowers this number by one. At this point the simplest route for us is to notice that we are looking for a particular solution that asymptotes to the extremal supersymmetric one found in [10],[13] (see, in particular, appendix B there). In that context most of our constants get fixed, as we require the same UV behavior; we use the expressions in that solution to fix six constants. Of the three constants left, one gets fixed by the asymptotic behavior of the non-extremality function, that is, we choose

$$
h(\rho) \sim 1-C_{2} e^{-\frac{8}{3} \rho} .
$$

The leading coefficient guarantees that we will have, after rotation, a solution with KT asymptotics. The key observation to be made is that, in principle, there is a mode allowed that we have explicitly set to zero. Namely, another solution to the equations of motion in the asymptotic regime could be

$$
h(\rho) \sim 1+C_{1} e^{-2 \rho}-C_{2} e^{-\frac{8}{3} \rho}+\ldots .
$$

The above expression should be thought of schematically as the solution to a second order differential equation for $h(\rho)$ which is defined asymptotically by two parameters. This $C_{1}$ is one of the three constants that we fix and therefore we remain with two constants that we called $\left(c, C_{2}\right)$. The parameter $c$ has the same meaning discussed in [10],[13] and $C_{2}$ is related to the temperature. Thus, the condition that the solution after the rotation has KT asymptotics plus a condition on the non-extremality function determine the values of the integration constants.

It is worth noting that selecting the right constants in the D3/D5 picture is more cumbersome as there are more parameters in the generic D3/D5 approach to black holes with KT asymptotics. For example, our solution does not allow for a generic mode that will lead to a Freund-Rubin form for $F_{5}$. As has been repeatedly emphasized, our $F_{5}$ is related to the non-extremality function and deforms the typical term proportional to the volume 5 -form. Let us explicitly visualize the difficulty in choosing the asymptotic expansion from the D3/D5 point of view in another example. The choice $C_{1}=0$ roughly corresponds to a dimension 3 operator in the D3/D5 picture. This can be seen easily by going to the radial coordinate $u=\exp (2 \rho / 3)$.

papers [10], [22] is such that the dilaton asymptotes to a (tunable) constant and the subleading terms are precisely those needed to generate the warp factor of Klebanov-Strassler 


\section{Conclusions and Future Directions}

We present here some concluding remarks together with some possible future projects to complement and refine the material in this paper.

In this work we have elaborated on the U-duality proposed in [6]. In that case, it was used to connect solutions describing wrapped D5 branes with the family of solutions describing the strong coupling dynamics of the baryonic branch of the Klebanov-Strassler field theory. In our case, we have used this U-duality to construct a new family of solutions describing the finite temperature phase of this baryonic branch. But before doing so, we explored the U-duality (that, following custom, we called rotation) in more generality, as a solution generating technique. We applied it to flat Dp branes in two different circumstances so that we appreciated the subtle differences making the choice of dualities in [6] so special.

Once we developed this technology, we applied it to rotate the Witten model for a Yang-Mills dual [17]. All this represents new material and it would be interesting to find an application for it, hopefully in the context of duals to Yang-Mills or QCD-like field theories.

In section 3, we discussed various new things: first a class of non-extremal solutions describing five branes wrapping a two-cycle inside the resolved conifold. The main feature of these non-extremal backgrounds is that the dilaton is asymptotically stabilized, which we interpreted as coupling the dual field theory to gravity modes. Then, we applied the U-duality to this solution, to find a new background of IIB that describes a non-extremal deformation of the Klebanov-Tseytlin solution (decoupled from gravity or stringy modes). We briefly commented on the associated thermodynamic properties of this new family of backgrounds. Finally, we discussed various aspects of this coupling/decoupling from gravity modes in section 4 .

Let us now list a set of projects that complement the material presented here and that may improve the understanding of the topic:

- It is important to sort out the details of the thermodynamics after the rotation in the solutions presented in section 3. Indeed, as we indicated in section 3.5, this poses a numerical problem, but certainly does not amount to a conceptual obstruction. The result for quantities like the specific heat or the free energy will be of great interest. Notice that this presents a novel way to approach the finite temperature phase of the Klebanov-Strassler field theory. Comparison with the solutions of [38] would be interesting.

- More conceptually; it would be nice to better understand the role played by the D0 branes in this solution generating technique. Indeed, notice the differences between the examples analyzed in section 2 and the one in section 2.1, all due to the presence of D0 branes before the boost that translates into the different final results. In the same vein, it would be good to have a better understanding on the reasons for the equivalence between the U-duality explained here and the rotation of the $J_{2}, \Omega_{3}$ forms as described 
in $[12],[13]$.

- It would be of very much interest to extend our non-extremal solution to a background of the form in eq.(3.28) where the restriction $a(\rho)=b(\rho)=0$ is not applied. Indeed, finding such a non-extremal solution would, after the application of the U-duality, produce a solution dual to a non-SUSY KS-like field theory in the baryonic branch. It may prove interesting to compare this new solution with that presented in the papers [35]. The different nature of the non-SUSY deformation will certainly reflect in different field theory aspects.

- It would be nice to use the solution presented in section 3 as a base to produce a non-SUSY deformation along the line proposed recently in [36]. The authors of [36] are finding SUSY breaking backgrounds by adding anti-D3 branes. Their solutions have singularities (and the problem is if such a singularity is physically acceptable). Proceeding as with the solution in this paper may set us on a different branch of solutions.

- It would be interesting to find a Physics application of the new backgrounds of section 2.1.1.

- It would be nice to extend the treatment here to the case in which the seed background contains $N_{f} \sim N_{c}$ flavors. This would be following the lead of the papers [24], [25]. The subtle point would be how to rotate such solutions (in other words, how the sources get represented in M-theory, see [37]. Comparison with the solutions of [38] would be interesting)

- We have presented two numerical solutions as examples of the type of backgrounds obtained. These examples correspond to specific values of the UV parameteers. We did not attempt a study of the full family of solutions in the whole parameter space. This is an interesting question worth investigating.

We hope to address some of these problems in the near future.

\section{Acknowledgments:}

We would like to thank various colleagues for the input that allowed us to better understand and present the results of this paper. We would like to single out Eloy Ayón-Beato, Iosif Bena, Alex Buchel, Pau Figueras, Gregory Giecold, Mariana Graña, Prem Kumar, Dario Martelli, Ioannis Papadimitriou, Michela Petrini, Dori Reichmann and César Terrero-Escalante. Very special thanks to Gregory Giecold for a very careful reading of the manuscript. E.C. thanks the Theory Group at the University of Texas at Austin for hospitality. 
This work was supported in part by the National Science Foundation under Grant Numbers PHY-0969020 and PHY-0455649, the US Department of Energy under grant DE-FG0295ER40899 and CONACyT grant CB-2008-01-104649.

\section{A Appendix: U-duality in the case of flat Dp-branes}

In this appendix we will give a sketchy derivation of the U-duality chain, in the case in which we rotate flat Dp-branes. We will not consider the non-extremal case, this can be done easily and the result of doing so is included in the first section of the paper. Here we want just to emphasize some points.

We start from the SUSY configuration in type II supergravity (string frame),

$$
\begin{aligned}
& d s^{2}=H^{-1 / 2}\left(-d t^{2}+d x_{p}^{2}\right)+H^{1 / 2}\left(d \rho^{2}+\rho^{2} d \Omega_{8-p}\right), \\
& e^{2 \phi}=H^{\frac{3-p}{2}}, F_{p+2}=\partial_{\rho} \hat{A} d t \wedge d x_{1} \wedge \ldots d x_{p} \wedge d \rho
\end{aligned}
$$

where $\hat{A}=-A$ in the notation of Section 2. Following standard convention we define the charge of $F_{8-p}$ as positive. The sign of $F_{p+2}$ will then depend on whether we consider Minkowski or Euclidean worldvolume on the $D_{p}$ brane. We apply T-duality in the $x_{p}$ directions,

$$
\begin{aligned}
d s^{2} & =-H^{-1 / 2} d t^{2}+H^{1 / 2}\left(d x_{p}^{2}+d \rho^{2}+\rho^{2} d \Omega_{8-p}\right), \\
e^{2 \phi} & =H^{\frac{3}{2}}, \quad F_{2}=\partial_{\rho} \hat{A} d t \wedge d \rho
\end{aligned}
$$

We lift this to M-theory, using $e^{\frac{4 \phi}{3}}=H$,

$$
d s_{11}^{2}=H\left(d x_{11}+\hat{A} d t\right)^{2}-H^{-1} d t^{2}+d M_{9}, d M_{9}=\left(d x_{p}^{2}+d \rho^{2}+\rho^{2} d \Omega_{8-p}\right)
$$

we now boost in the $t-x_{11}$ direction with rapidity $\beta$

$$
d t \rightarrow \cosh \beta d t-\sinh \beta d x_{11}, \quad d x_{11} \rightarrow-\sinh \beta d t+\cosh \beta d x_{11},
$$

to get

$$
\begin{aligned}
& d s_{11}^{2}=A d t^{2}+B d x_{11}^{2}+C d t d x_{11}+d M_{9} \\
& A=H^{-1}\left[H^{2}(\hat{A} \cosh \beta-\sinh \beta)^{2}-\cosh ^{2} \beta\right] \\
& B=H^{-1}\left[H^{2}(\hat{A} \sinh \beta-\cosh \beta)^{2}-\sinh ^{2} \beta\right] \\
& C=-2 H^{-1}\left[H^{2}(\hat{A} \cosh \beta-\sinh \beta)(\hat{A} \sinh \beta-\cosh \beta)-\cosh \beta \sinh \beta\right]
\end{aligned}
$$

and we prepare the metric for reduction back to IIA as

$$
\begin{aligned}
& d s_{11}^{2}=B\left(d x_{11}-a_{t} d t\right)^{2}+B^{-1 / 2}\left[g_{t t} d t^{2}+B^{1 / 2} d M_{9}\right], \\
& a_{t}=-\frac{C}{2 B}, \quad g_{t t}=\frac{4 A B-C^{2}}{4 \sqrt{B}}
\end{aligned}
$$


we now write the IIA configuration as,

$$
d s_{I I A}^{2}=g_{t t} d t^{2}+B^{1 / 2}\left(d x_{p}^{2}+d \rho^{2}+\rho^{2} d \Omega_{8-p}\right), e^{2 \phi}=B^{3 / 2}, F_{2}=\partial_{\rho} a_{t} d t \wedge d \rho
$$

and we T-dualize back in the $x_{p}$ directions to get

$$
\begin{aligned}
& d s_{I I}^{2}=g_{t t} d t^{2}+B^{-1 / 2} d x_{p}^{2}+B^{1 / 2}\left(d \rho^{2}+\rho^{2} d \Omega_{8-p}\right), \\
& e^{2 \phi}=B^{\frac{3-p}{2}}, \quad F_{p+2}=\partial_{\rho} a_{t} d t \wedge d \rho \wedge d x_{1} \ldots \wedge d x_{p}
\end{aligned}
$$

We see that the $S O(1, p)$ invariance is preserved, as $g_{t t} \sqrt{B}=-1$.

\section{B Appendix: Black Dp branes}

In this appendix we gather some useful facts about black Dp-brane solutions [26],[27],[28]. The metric in string frame is,

$$
d s^{2}=-\frac{f_{+}(r)}{\sqrt{f_{-}(r)}} d t^{2}+\sqrt{f_{-}(r)} \sum_{i=1}^{p} d x^{i} d x^{i}+\frac{f_{-}(r)^{-\frac{1}{2}-\frac{5-p}{7-p}}}{f_{+}(r)} d r^{2}+r^{2} f_{-}(r)^{\frac{1}{2}-\frac{5-p}{7-p}} d \Omega_{8-p}^{2}
$$

where

$$
f_{ \pm}(r)=1-\left(\frac{r_{ \pm}}{r}\right)^{7-p}
$$

The mass per unit volume $M$ and the R-R charge $N$ are

$$
M=\frac{1}{(7-p)(2 \pi)^{7} d_{p} l_{P}^{8}}\left((8-p) r_{+}^{7-p}-r_{-}^{7-p}\right), \quad N=c_{p}\left(r_{+} r_{-}\right)^{\frac{7-p}{2}},
$$

where $l_{P}$ is the ten dimensional Planck length $l_{P}=g_{s}^{1 / 4} l_{s}$ and $c_{p}, d_{p}$ are numerical factors,

$$
\begin{aligned}
& d_{p}=2^{5-p} \pi^{\frac{5-p}{2}} \Gamma\left(\frac{7-p}{2}\right) \\
& c_{p}=\frac{1}{d_{p} g_{s} l_{s}^{7-p}}=\frac{(7-p) V o l_{S^{8-p}}}{\left(2 \pi l_{s}\right)^{7-p} g_{s}}
\end{aligned}
$$

The background also has a nontrivial dilaton and a R-R flux,

$$
\begin{aligned}
e^{-2 \Phi} & =g_{s}^{-2} f_{-}(r)^{-\frac{p-3}{2}} \\
\frac{1}{\left(2 \pi l_{s}\right)^{7-p}} \int_{S^{8-p}} * F_{p+2} & =N .
\end{aligned}
$$

The Einstein frame metric $\left(g_{E_{\mu \nu}}=\sqrt{g_{s} e^{-\Phi}} g_{s t_{\mu \nu}}\right)$ has a horizon at $r=r_{+}$and, for $p \leq 6$, a singularity at $r=r_{-}$. If $r_{+}>r_{-}$the singularity is covered by the horizon and the solution is a black hole. In the extremal case, $r_{+}=r_{-}$, the space is singular except for $p=3$. 
Let us define a new coordinate,

$$
\rho^{7-p}=r^{7-p}-r_{-}^{7-p}
$$

. This change of variables transforms (B.1) to a more familiar form,

$$
d s^{2}=H(\rho)^{-\frac{1}{2}}\left(-h(\rho) d t^{2}+\sum_{i=1}^{p} d x^{i} d x^{i}\right)+H(\rho)^{\frac{1}{2}}\left(\frac{d \rho^{2}}{h(\rho)}+\rho^{2} d \Omega_{8-p}^{2}\right)
$$

where

$$
\begin{aligned}
& H(\rho)=1+\frac{r_{-}^{7-p}}{\rho^{7-p}} \\
& h(\rho)=1-\frac{r_{+}^{7-p}-r_{-}^{7-p}}{\rho^{7-p}}
\end{aligned}
$$

Throughout the paper we use the notation,

$$
L_{p}^{7-p} \equiv r_{-}^{7-p} \quad \text { and } \quad R_{T}^{7-p} \equiv r_{+}^{7-p}-r_{-}^{7-p}
$$

Note that using (B.3) one can also write

$$
\begin{aligned}
L_{p}^{7-p} & =\tilde{Q}\left[\sqrt{1+\left(\frac{R_{T}^{7-p}}{2 \tilde{Q}}\right)^{2}}-\frac{R_{T}^{7-p}}{2 \tilde{Q}}\right] \\
& =\tilde{Q} \alpha_{p} .
\end{aligned}
$$

where $\tilde{Q}=\frac{N}{c_{p}}$ and $\alpha_{p}=\left(1+\left(\frac{R_{T}^{7-p}}{2 \tilde{Q}}\right)^{2}\right)^{1 / 2}-\frac{R_{T}^{7-p}}{2 \tilde{Q}}$.

¿From (B.3) we can see that the parameter $R_{T}$ is related to the energy per unit volume above extremality $\epsilon$,

$$
\Delta M=\epsilon=\frac{r_{+}^{7-p}-r_{-}^{7-p}}{(2 \pi)^{7} d_{p} l_{P}^{8}}=\frac{R_{T}{ }^{7-p}}{(2 \pi)^{7} d_{p} l_{P}^{8}} .
$$

Note that in the decoupling limit $\left(\alpha^{\prime} \rightarrow 0\right.$, energies fixed $) \epsilon$ remains fixed and $\alpha_{p} \rightarrow 1$.

In the extremal case, $r_{+}=r_{-}$, we have $R_{T}=0$ and $L_{p}^{7-p}=\tilde{Q}=N d_{p} g_{s} l_{s}^{7-p}$.

\section{Appendix: Some details on the U-duality}

Here we provide details for the derivations of eq.(2.24). We started with

$$
\begin{aligned}
& d s^{2}=H^{-1 / 2}\left[-d t^{2}+d x_{p-q}^{2}+d \sigma_{q}^{2}\right]+H^{1 / 2}\left[d \rho^{2}+\rho^{2} d \Omega_{8-p}^{2}\right], \\
& F_{p+2}=\partial_{\rho} \hat{A} d t \wedge d x_{1} \wedge \ldots \wedge d x_{p-q} \wedge d \sigma_{q} \wedge d \rho, \\
& e^{2 \phi[\text { initial }]}=e^{2 \phi(\infty)} H^{\frac{3-p}{2}} .
\end{aligned}
$$


The we perform the U-duality described in the text below eq.(2.23)

$$
\begin{aligned}
& d s^{2}=H^{-1 / 2}\left[-d t^{2}+d \sigma_{q}^{2}\right]+H^{1 / 2}\left[d \rho^{2}+\rho^{2} d \Omega_{8-p}^{2}+d x_{p-q}^{2}\right] \\
& F_{q+2}=\partial_{r} \hat{A} d t \wedge d \sigma_{q} \wedge d \rho \\
& e^{2 \phi}=e^{2 \phi(\infty)} H^{\frac{3-q}{2}}
\end{aligned}
$$

Now, we need this to be a configuration in Type IIA (in order to lift this to M-theory). We will also want to impose that when lifted to M-theory this will produce a four-form field $G_{4}$. If this is the case, we must have that either $q=2$ or that $q=4$ (the cases of $q=0, q=6$ are analogous to what we analyzed in the first section). The case $q=2$, on which we will elaborate upon below, has the peculiarity that the reduction from eleven dimensions back to IIA will generate a NS $H_{3}$ field, proportional to the boost. Let us see this in detail. To begin with, we will lift to eleven dimensions the configuration in eq.(C.2).

$$
\begin{aligned}
& d s_{11}^{2}=H^{1 / 3} d x_{11}^{2}+H^{-2 / 3}\left[-d t^{2}+d \vec{\sigma}_{2}^{2}\right]+H^{1 / 3}\left[d \rho^{2}+\rho^{2} d \Omega_{8-p}^{2}+d \vec{x}_{p-2}^{2}\right] \\
& G_{4}=\partial_{\rho} \hat{A} d t \wedge d \rho \wedge d \sigma_{1} \wedge d \sigma_{2}
\end{aligned}
$$

and now we boost with rapidity $\beta$

$$
d t \rightarrow \cosh \beta d t-\sinh \beta d x_{11}, \quad d x_{11} \rightarrow-\sinh \beta d t+\cosh \beta d x_{11}
$$

and we can rewrite the configuration after the boost as,

$$
\begin{aligned}
& d s_{11}^{2}=A d t^{2}+B d x_{11}^{2}+C d t d x_{11}+d M_{9}^{2}, \\
& G_{4}=\partial_{\rho} \hat{A}\left(\cosh \beta d t-\sinh \beta d x_{11}\right) \wedge d \rho \wedge d \sigma_{1} \wedge d \sigma_{2}, \\
& d M_{9}^{2}=H^{-2 / 3} d \vec{\sigma}_{2}^{2}+H^{1 / 3}\left[d \rho^{2}+\rho^{2} d \Omega_{8-p}^{2}+d \vec{x}_{p-2}^{2}\right], \\
& A=H^{-2 / 3}\left[H \sinh ^{2} \beta-\cosh ^{2} \beta\right] \\
& B=H^{-2 / 3}\left[H \cosh ^{2} \beta-\sinh ^{2} \beta\right] \\
& C=2 H^{-2 / 3} \sinh \beta \cosh \beta(1-H)
\end{aligned}
$$

when we reduce this to IIA we get,

$$
\begin{aligned}
& d s_{I I A, s t}^{2}=g_{t t} d t^{2}+B^{1 / 2}\left(H^{-2 / 3} d \vec{\sigma}_{2}^{2}+H^{1 / 3}\left[d \rho^{2}+\rho^{2} d \Omega_{8-p}^{2}+d \vec{x}_{p-2}^{2}\right]\right) \\
& e^{2 \phi[A]}=B^{3 / 2}, \quad g_{t t}=\frac{4 A B-C^{2}}{4 \sqrt{B}} \\
& F_{4}=\partial_{\rho} \hat{A}\left(\cosh \beta+a_{t} \sinh \beta\right) d t \wedge d \rho \wedge d \sigma_{1} \wedge d \sigma_{2} \\
& H_{3}=\sinh \beta \partial_{\rho} \hat{A} d \rho \wedge d \sigma_{1} \wedge d \sigma_{2}, \\
& F_{2}=\partial_{\rho}\left(a_{t}\right) d t \wedge d \rho, \quad a_{t}=\frac{C}{2 B}=\frac{\sinh \beta \cosh \beta(1-H)}{H \cosh \beta-\sinh ^{2} \beta}
\end{aligned}
$$


we see that we have generated a NS magnetic field. Finally, we T-dualize back in the $\vec{x}_{p-1}$ directions, to get

$$
\begin{aligned}
& d s_{I I, s t}^{2}=g_{t t} d t^{2}+\frac{d \vec{x}_{p-2}^{2}}{H^{1 / 3} B^{1 / 2}}+B^{1 / 2}\left(H^{-2 / 3} d \vec{\sigma}_{2}^{2}+H^{1 / 3}\left[d \rho^{2}+\rho^{2} d \Omega_{8-p}^{2}\right]\right), \\
& F_{p+2}=\partial_{\rho} \hat{A}\left(\cosh \beta+a_{t} \sinh \beta\right) \wedge d \rho \wedge d \sigma_{1} \wedge d \sigma_{2} \wedge d x_{1} \wedge \ldots \wedge d x_{p-2}, \\
& H_{3}=\sinh \beta \partial_{\rho} \hat{A} d \rho \wedge d \sigma_{1} \wedge d \sigma_{2}, \\
& F_{p}=\partial_{\rho}\left(a_{t}\right) d t \wedge d \rho \wedge d x_{1} \wedge \ldots \wedge d x_{p-2}, \\
& e^{2 \phi[\text { final }]}=B^{\frac{5-p}{2}} H^{\frac{2-p}{3}} .
\end{aligned}
$$

This completes our derivation of eq.(2.24).

\section{Appendix: Another solution generating algorithm}

We studied two different 'solution generating techniques' and applied them to different backgrounds. All these 'algorithms' were starting with a background solution to the Type II (A or B) equations of motion, applying a number of T-dualities that would transform the background into a solution for Type IIA supergravity. Then we lifted this to eleven dimensions, where a boost was applied (inducing a one parameter- $\beta$ - family of solutions), then reducing to IIA and T-dualizing back we had our final generated background.

One may wonder what is the algorithm when, after a number of T-dualities, we end with a background solving the Type IIB supergravity equations of motion. In this case, a way of generating a one-parameter family of solutions may be S-dualizing. Indeed, given $a, b, c, d$ real numbers satisfying $a d-b c=1$, we can start with a IIB solution having axion $\chi$, dilaton $\phi, \mathrm{RR}$ and NS thre forms $F_{3}=d C_{2}$ and $H_{3}=d B_{2}$ and five form $F_{5}=d C_{4}+F_{3} \wedge B_{2}$ and by S-duality, generate a new solutions dependent on the three independent real parameters $a, b, c$. Indeed, the five form is left invariant and the same happens for the Einstein frame metric, while

$$
\begin{aligned}
& F_{3}[\text { new }]=b H_{3}+a F_{3}, \quad H_{3}[\text { new }]=d H_{3}+c F_{3}, \\
& e^{\phi[n e w]}=\left((c \chi+d)^{2}+c^{2} e^{-2 \phi}\right) e^{\phi}, \quad \chi[n e w]=\frac{(a \chi+b)(c \chi+d)+a c e^{-2 \phi}}{\left((c \chi+d)^{2}+c^{2} e^{-2 \phi}\right)}
\end{aligned}
$$

Let us focus our attention in the particular set of values $a=d=0, c b=-1$, this is the transformation that interchanges the three forms and inverts the value of the dilaton (if the axion is initially zero, as we will assume).

One can then think about applying this transformation expecting to generate new interesting solutions. As an example, suppose that we start with solutions describing D6 branes wrapping a three-cycle inside the deformed conifold. Those solutions are dual to a (UV completed version of) $\mathrm{N}=1 \mathrm{SYM}$ [29]. A particularly interesting solution is given in section 3.2 of 
the paper [30]. The background consist of metric, dilaton and RR two-form field,

$$
d s_{I I A, s t}^{2}=e^{f}\left[-d t^{2}+d x_{1}^{2}+d x_{2}^{2}+d x_{3}^{2}+d M_{6}^{2}\right], \quad e^{2 \phi}=e^{3 f+2 \phi_{0}}, \quad F_{2}=d A_{1}
$$

where all the details (the manifold $M_{6}$, the functional form of $f, \phi$ and the one form $A_{1}$ are given in eqs(57)-(59) of [30]). We can the perform three T-dualities in $x_{1,2,3}$ leading to

$$
\begin{aligned}
& d s_{I I B, s t}^{2}=e^{f}\left[-d t^{2}+d M_{6}^{2}\right]+e^{-f} d x_{1,2,3}^{2}, \quad e^{2 \phi}=e^{2 \phi_{0}}, \\
& F_{5}=d A_{1} \wedge d x_{1} \wedge d x_{2} \wedge d x_{3}
\end{aligned}
$$

that is we have generated D3 brane charge and the dilaton is just a constant. We now move this to Einstein frame, that leaves us-up to a constant-with the same metric, perform the Sduality metioned above and T-dualize back. This brings us to the starting point background $(D .2)^{10}$. Something similar occurs if we start with a solution describing D5 branes wrapping a three-cycle inside a G2 holonomy manifold [31], a configuration dual to N=1 Yang-Mills ChernSimons in $2+1$ dimensions (with its respective UV completion). Notice that this operation is not the one proposed in [20], that is the reason why these authors were able to generate an interesting solution starting from [31].

In both these cases described above, we are generating charge of D3 brane, this is invariant under the S-duality, hence it is expected that the whole operation brings us back to the initial configuration. A more interesting example is to start from the configuration of D4 branes wrapping a circle with SUSY breaking boundary conditions [17] that we discussed before, see Section 2.1.1.

In this case we will apply three T-dualities to a IIA configuration. This will generate charge of D1 brane that the S-duality will interchange with F1 charge, after T-dualizing back, we will generate a new background (the $S O(1,3)$ isometry will be spoiled, so we may want to start with the high Temperature dual, hence having a non-extremal factor $h(\rho)$ in front of $d t^{2}$ and the function $f(\rho)=1$ in front of $d x_{4}^{2}$, but let us keep things general). Let us see some details. After the T-dualities, the configuration reads

$$
\begin{aligned}
& d s_{I I B, s t}^{2}=H^{-1 / 2}\left(-h d t^{2}+f d x_{4}^{2}\right)+H^{1 / 2}\left(\frac{d \rho^{2}}{h}+\rho^{2} d \Omega_{4}^{2}+d x_{1,2,3}^{2}\right), \\
& e^{2 \phi}=g_{s}^{2} H, \quad F_{3}=\partial_{\rho} A d t \wedge d x_{4} \wedge d \rho
\end{aligned}
$$

now, we need to move this to Einstein frame, multiplying the metric by $e^{-\frac{\phi}{2}}$, perform the S-duality that will generate $H_{3}$ and a dilaton $e^{-2 \phi}=g_{s}^{2} H$. Then, T-dualize back in $x_{1,2,3}$. The final configurations is,

$$
\begin{aligned}
& d s_{I I A, s t}^{2}=\frac{c^{1 / 4}}{g_{s} H}\left(-h d t^{2}+f d x_{4}^{2}\right)+\frac{d \rho^{2}}{h}+\rho^{2} d \Omega_{4}^{2}+d x_{1,2,3}^{2}, \\
& H_{3}=c \partial_{\rho} A d t \wedge d x_{4} \wedge d \rho, \quad c^{4} e^{-2 \phi}=g_{s}^{2} H
\end{aligned}
$$

\footnotetext{
${ }^{10}$ One may perform an S-duality with parameters $a=0, b c=-1$ and $d$ being free. This will generate a background in IIB with constant axion-dilaton. The final IIA configuration is the same as the initial one.
} 
As a final remark; had we chosen to T-dualize only in the $x_{4}$ direction (in the supergravity approximation, this can be done in the high Temperature phase only), we would have generated D3 branes, hence after the S-duality and T-duality, we would be back to the initial configuration.

\section{E Appendix: U-duality for the wrapped D5 branes Black Hole}

We will describe in detail the action of the solution generating technique proposed in [6], when applied to the background of eq.(3.30). These techniques have been applied in a similar context in [39]. Let us consider things in the string frame.

$$
\begin{aligned}
& d s_{s}^{2}=e^{\phi}\left[-h(\rho) d t^{2}+d x_{1}^{2}+d x_{2}^{2}+d x_{3}^{2}\right]+d s_{6, s}^{2} \\
& d s_{6}^{2}=e^{\phi}\left[\frac{e^{2 k}}{s(\rho)} d \rho^{2}+\frac{e^{2 k}}{4}\left(\tilde{\omega}_{3}+\cos \theta d \varphi\right)^{2}+e^{2 q}\left(d \theta^{2}+\sin ^{2} \theta d \varphi^{2}\right)+\frac{e^{2 g}}{4}\left(d \tilde{\theta}^{2}+\sin ^{2} \tilde{\theta} d \tilde{\varphi}^{2}\right)\right] \\
& F_{(3)}=\frac{N_{c}}{4}\left[-\tilde{\omega}_{1} \wedge \tilde{\omega}_{2}+\sin \theta d \theta \wedge d \varphi\right] \wedge\left(\tilde{\omega}_{3}+\cos \theta d \varphi\right)
\end{aligned}
$$

where $h(\rho), s(\rho)$ are the non-extremality functions and we will choose $h(\rho)=s(\rho)$ as we did in section 3. We can proceed to rotate it. We will follow the procedure explained in [6]. So, let us start by writing the effect of the first T-duality in the $x_{1}$ direction (all the expressions below are in string frame)

$$
\begin{aligned}
& d s_{I I A}^{2}=e^{f}\left[-h d t^{2}+d x_{2}^{2}+d x_{3}^{2}\right]+e^{-f} d x_{1}^{2}+d s_{6}^{2}, \\
& e^{2 \phi_{A}}=e^{2 \phi-f}, \quad F_{4}=F_{3} \wedge d x_{1}
\end{aligned}
$$

The function $f=\phi$ will be kept to avoid confusion with the transformed dilatons. Now, we perform the T-duality in $x_{2}$

$$
\begin{aligned}
& d s_{I I A}^{B}=e^{f}\left[-h d t^{2}+d x_{3}^{2}\right]+e^{-f}\left(d x_{1}^{2}+d x_{2}^{2}\right)+d s_{6}^{2}, \\
& e^{2 \phi_{B}}=e^{2 \phi-2 f}, \quad F_{5}=F_{3} \wedge d x_{1} \wedge d x_{2}\left(1+*_{10}\right)
\end{aligned}
$$

T-dualizing in $x_{3}$, we get

$$
\begin{aligned}
& d s_{I I A}^{2}=e^{f}\left[-h d t^{2}\right]+e^{-f}\left(d x_{1}^{2}+d x_{2}^{2}+d x_{3}^{2}\right)+d s_{6}^{2}, \\
& e^{2 \phi_{A}}=e^{2 \phi-3 f}, \\
& F_{6}=F_{3} \wedge d x_{1} \wedge d x_{2} \wedge d x_{3} \rightarrow F_{4}=e^{2 f} \sqrt{h} *_{6} F_{3} \wedge d t
\end{aligned}
$$


Notice that

$$
*_{6} F_{3}=\frac{N_{c}}{\sqrt{h}}\left[-2 e^{2 q-2 g} \sin \theta d \theta \wedge d \varphi+\frac{e^{2 g-2 q}}{8} \sin \tilde{\theta} d \tilde{\theta} \wedge d \tilde{\varphi}\right] \wedge d \rho .
$$

The factor of $\sqrt{h}$ in the $F_{4}$ of eq.(E.4) is present to cancel the factor of $\sqrt{h}$ in the denominator of eq.(E.5). Now, we lift this to M-theory;

$$
\begin{aligned}
& d s_{11}^{2}=e^{4 / 3 \phi-2 f} d x_{11}^{2}+e^{f-2 / 3 \phi}\left[-h e^{f} d t^{2}+e^{-f}\left(d x_{1}^{2}+d x_{2}^{2}+d x_{3}^{2}\right)+d s_{6}^{2}\right], \\
& G_{4}=\sqrt{h} e^{2 f} *_{6} F_{3} \wedge d t .
\end{aligned}
$$

We boost in the $t-x_{11}$ directions according to,

$$
d t \rightarrow \cosh \beta d t-\sinh \beta d x_{11}, \quad d x_{11} \rightarrow-\sinh \beta d t+\cosh \beta d x_{11}
$$

and now we rewrite this boosted metric as,

$$
\begin{aligned}
& d s_{11}^{2}=e^{f-2 / 3 \phi}\left[e^{-f}\left(d x_{1}^{2}+d x_{2}^{2}+d x_{3}^{2}\right)+d s_{6}^{2}\right]+A d t^{2}+B d x_{11}^{2}+C d t d x_{11}, \\
& G_{4}=\sqrt{h} e^{2 f} *_{6} F_{3}\left[\cosh \beta d t-\sinh \beta d x_{11}\right]
\end{aligned}
$$

where,

$$
\begin{aligned}
& A=e^{2 f-2 / 3 \phi}\left[\sinh ^{2} \beta e^{2 \phi-4 f}-h \cosh ^{2} \beta\right], \quad B=e^{2 f-2 / 3 \phi}\left[\cosh ^{2} \beta e^{2 \phi-4 f}-h \sinh ^{2} \beta\right], \\
& C=-2 \cosh \beta \sinh \beta e^{2 f-2 / 3 \phi}\left[e^{2 \phi-4 f}-h\right] .
\end{aligned}
$$

Now, we will reduce this to IIA, before doing so and in order to reduce to IIA, it is useful to rewrite eq.(E.8) as,

$$
\begin{aligned}
& d s_{11}^{2}=B^{-1 / 2}\left[g_{t t} d t^{2}+B^{1 / 2} e^{f-2 / 3 \phi}\left(e^{-f}\left(d x_{1}^{2}+d x_{2}^{2}+d x_{3}^{2}\right)+d s_{6}^{2}\right)\right]+B\left(d x_{11}+a_{t} d t\right)^{2}, \\
& G_{4}=\sqrt{h} e^{2 f} *_{6} F_{3}\left[\left(\cosh \beta+a_{t} \sinh \beta\right) d t-\sinh \beta\left(d x_{11}+a_{t} d t\right)\right]
\end{aligned}
$$

where we have defined

$$
a_{t}=\frac{C}{2 B}, \quad g_{t t}=\frac{4 A B-C^{2}}{4 \sqrt{B}}, \quad e^{4 / 3 \phi_{A}}=B .
$$

Now, we reduce to IIA, obtaining in string frame,

$$
\begin{aligned}
& d s_{I I A}^{2}=g_{t t} d t^{2}+\sqrt{B} e^{-2 / 3 \phi}\left(d x_{1}^{2}+d x_{2}^{2}+d x_{3}^{2}\right)+\sqrt{B} e^{f-2 / 3 \phi} d s_{6}^{2}, \\
& e^{2 \phi_{A}}=B^{3 / 2}, \\
& F_{4}=\sqrt{h} e^{2 f} *_{6} F_{3} \wedge\left[\left(\cosh \beta+a_{t} \sinh \beta\right) d t\right], \\
& H_{3}=-\sinh \beta \sqrt{h} e^{2 f} *_{6} F_{3}, \\
& F_{2}=a_{t}^{\prime} d \rho \wedge d t
\end{aligned}
$$


Now, we proceed to do the T-dualities back; T-dualizing in the $x_{3}$ direction we have

$$
\begin{aligned}
& d s_{I I B}^{2}=g_{t t} d t^{2}+\sqrt{B} e^{-2 / 3 \phi}\left(d x_{1}^{2}+d x_{2}^{2}\right)+\frac{e^{2 / 3 \phi}}{\sqrt{B}} d x_{3}^{2}+\sqrt{B} e^{f-2 / 3 \phi} d s_{6}^{2}, \\
& e^{2 \phi_{B}}=B e^{2 / 3 \phi}, \\
& F_{5}=\sqrt{h} e^{2 f} *_{6} F_{3} \wedge\left[\left(\cosh \beta+a_{t} \sinh \beta\right) d t \wedge d x_{3}\right]\left(1+*_{10}\right), \\
& H_{3}=-\sinh \beta \sqrt{h} e^{2 f} *_{6} F_{3}, \\
& F_{3}=a_{t}^{\prime} d \rho \wedge d t \wedge d x_{3}
\end{aligned}
$$

now, we T-dualize in $x_{2}$

$$
\begin{aligned}
& d s_{I I A}^{2}=g_{t t} d t^{2}+\sqrt{B} e^{-2 / 3 \phi}\left(d x_{1}^{2}\right)+\frac{e^{2 / 3 \phi}}{\sqrt{B}}\left(d x_{3}^{2}+d x_{2}^{2}\right)+\sqrt{B} e^{f-2 / 3 \phi} d s_{6}^{2}, \\
& e^{2 \phi_{A}}=\sqrt{B} e^{4 / 3 \phi}, \\
& F_{6}=\sqrt{h} e^{2 f} *_{6} F_{3} \wedge\left[\left(\cosh \beta+a_{t} \sinh \beta\right) d t\right] \wedge d x_{3} \wedge d x_{2}, \\
& H_{3}=-\sinh \beta \sqrt{h} e^{2 f} *_{6} F_{3}, \\
& F_{4}=a_{t}^{\prime} d \rho \wedge d t \wedge d x_{3} \wedge d x_{2}
\end{aligned}
$$

finally, we T-dualize in $x_{1}$

$$
\begin{aligned}
& d s_{I I B}^{2}=g_{t t} d t^{2}+\frac{e^{2 / 3 \phi}}{\sqrt{B}}\left(d x_{3}^{2}+d x_{1}^{2}+d x_{2}^{2}\right)+\sqrt{B} e^{f-2 / 3 \phi} d s_{6}^{2} \\
& e^{2 \phi_{B}}=e^{2 \phi} \\
& F_{7}=\sqrt{h} e^{2 f} *_{6} F_{3} \wedge\left[\left(\cosh \beta+a_{t} \sinh \beta\right) d t\right] \wedge d x_{3} \wedge d x_{2} \wedge d x_{1} \quad F_{3}=*_{10} F_{7}, \\
& H_{3}=-\sinh \beta \sqrt{h} e^{2 f} *_{6} F_{3}, \\
& F_{5}=a_{t}^{\prime} d \rho \wedge d t \wedge d x_{3} \wedge d x_{2} \wedge d x_{1}\left(1+*_{10}\right) .
\end{aligned}
$$

After using $f=\phi$ and the definitions for $A, B, C, a_{t}$ this encodes the result of eq.(3.35).

\section{F Appendix: The equations of motion}

In this appendix we will quote the equations of motion that we are numerically solving. Our goal is to find a black hole of the metrics described in the main text. In [23] the authors studied a very general Ansatz for non-extremal deformations of NS5 branes wrapped on $S^{2}$. Their Ansatz can be adapted to our case. It reads -in Einstein frame,

$$
d s^{2}=-Y_{1} d t^{2}+Y_{2} d \mathrm{x}^{n} d \mathrm{x}^{n}+Y_{3} d \rho^{2}+Y_{4}\left(e_{1}^{2}+e_{2}^{2}\right)+Y_{5}\left(\left(w^{1}\right)^{2}+\left(w^{2}\right)^{2}\right)+Y_{6}\left(w^{3}+A\right)^{2}
$$


Inserting this ansatz in the supergravity action we get,

$$
L=\sum_{i, j} G_{i j}(Y) Y_{i}^{\prime} Y_{j}^{\prime}-U(Y)=T-U
$$

Using a parametrization to make $G_{i j}$ diagonal and choosing the appropiate gauge to make contact with our ansatz we have,

$$
\begin{aligned}
T & =e^{2(g+q-4 x+\Phi)} \frac{1}{8}\left(\frac{1}{2}\left(g^{\prime 2}+q^{\prime 2}-2 \Phi^{\prime 2}\right)+2\left(g^{\prime} q^{\prime}-k^{\prime} x^{\prime}\right)+\left(g^{\prime}+q^{\prime}+\Phi^{\prime}\right)\left(k^{\prime}-4 x^{\prime}+2 \Phi^{\prime}\right)\right) \\
U & =\frac{1}{256} e^{-2(g+q-\Phi)}\left(-16 e^{2(g+q+k)}\left(e^{2 g}+4 e^{2 q}\right)+\left(e^{4 g}+16 e^{4 q}\right)\left(1+e^{4 k}\right)\right) .
\end{aligned}
$$

and,

$$
\begin{array}{lcc}
Y_{1}=e^{\Phi / 2} e^{-8 x}, & Y_{2}=e^{\Phi / 2}, & Y_{3}=e^{\Phi / 2} e^{8 x} e^{2 k}, \\
Y_{4}=e^{\Phi / 2} e^{2 g}, & Y_{5}=e^{\Phi / 2} e^{2 q} . & Y_{6}=\Phi
\end{array}
$$

¿From (F.2) we get the second order equations to solve,

$$
\begin{aligned}
& 2 e^{-4 g+8 x}+\frac{1}{8} e^{-4 q+8 x}-2 \Phi^{\prime}\left(g^{\prime}+q^{\prime}-4 x^{\prime}+\Phi^{\prime}\right)-\Phi^{\prime \prime}=0 \\
& -2 x^{\prime}\left(g^{\prime}+q^{\prime}-4 x^{\prime}+\Phi^{\prime}\right)-x^{\prime \prime}=0 \\
& -e^{4 k+8 x}\left(2 e^{-4 g}+\frac{1}{8} e^{-4 q}\right)+e^{8 x}\left(2 e^{-4 g}+\frac{1}{8} e^{-4 q}\right)+2 k^{\prime}\left(g^{\prime}+q^{\prime}-4 x^{\prime}+\Phi^{\prime}\right)+k^{\prime \prime}=0 \\
& 2 e^{8 x}\left(e^{4 k-4 g}-2 e^{2(k-g)}+1\right)+g^{\prime}\left(g^{\prime}+q^{\prime}-4 x^{\prime}+\Phi^{\prime}\right)+g^{\prime \prime}=0 \\
& e^{8 x}\left(-\frac{1}{8} e^{4 k-4 q}+e^{2 k-2 q}-\frac{1}{8} e^{-4 q}\right)-2 q^{\prime}\left(g^{\prime}+q^{\prime}-4 x^{\prime}+\Phi^{\prime}\right)-q^{\prime \prime}=0
\end{aligned}
$$

and a first order constraint which is a consequence of reparametrization invariance,

$$
\begin{aligned}
& \frac{e^{8 x}}{2}\left(\frac{1}{16}\left(e^{-4 q}+16 e^{-4 g}\right)\left(1+e^{4 k}\right)-e^{2 k}\left(e^{-2 q}+4 e^{-2 g}\right)\right)+\frac{1}{2}\left(g^{\prime 2}+q^{2}-2 \Phi^{\prime 2}\right)+ \\
& 2\left(g^{\prime} q^{\prime}-k^{\prime} x^{\prime}\right)+\left(g^{\prime}+q^{\prime}+\Phi^{\prime}\right)\left(k^{\prime}-4 x^{\prime}+2 \Phi^{\prime}\right)=0
\end{aligned}
$$

The second equation in (F.5) can be integrated to yield a first order equation and (F.5) becomes

$$
\begin{aligned}
2 e^{-4 g+8 x}+\frac{1}{8} e^{-4 q+8 x}-2 \Phi^{\prime}\left(g^{\prime}+q^{\prime}-4 x^{\prime}+\Phi^{\prime}\right)-\Phi^{\prime \prime} & =0 \\
x^{\prime}-\alpha e^{-2(g+q-4 x+\Phi)} & =0 \\
-e^{4 k+8 x}\left(2 e^{-4 g}+\frac{1}{8} e^{-4 q}\right)+e^{8 x}\left(2 e^{-4 g}+\frac{1}{8} e^{-4 q}\right)+2 k^{\prime}\left(g^{\prime}+q^{\prime}-4 x^{\prime}+\Phi^{\prime}\right)+k^{\prime \prime} & =0 \\
e^{8 x}\left(e^{4 k-4 g}-2 e^{2(k-g)}+1\right)+g^{\prime}\left(g^{\prime}+q^{\prime}-4 x^{\prime}+\Phi^{\prime}\right)+g^{\prime \prime} & =0 \\
e^{8 x}\left(-\frac{1}{8} e^{4 k-4 q}+e^{2 k-2 q}-\frac{1}{8} e^{-4 q}\right)-2 q^{\prime}\left(g^{\prime}+q^{\prime}-4 x^{\prime}+\Phi^{\prime}\right)-q^{\prime \prime} & =0
\end{aligned}
$$


where $\alpha$ is a non-extremality parameter.

One can verify that the transformation

$$
r \rightarrow e^{2 d} r \quad \Phi \rightarrow \Phi+d \quad x \rightarrow x+\frac{d}{2} \quad g \rightarrow g \quad q \rightarrow q \quad k \rightarrow k
$$

where $d$ is a constant, leaves (F.5) and (F.6) invariant and thus, is a symmetry of the equations of motion and constraint. Another, obvious, symmetry of (F.5) and ( F.6) is

$$
\Phi \rightarrow \Phi+C \quad \alpha \rightarrow \alpha e^{2 C}
$$

with all the other functions unchanged and $C$ a constant. Finally, there is also a translational symmetry

$$
r \rightarrow r+r_{0}
$$

that leaves all equations unchanged. Note that thermodynamic quantities should be invariant under all these symmetries.

\section{References}

[1] J. M. Maldacena, Adv. Theor. Math. Phys. 2, 231 (1998) [Int. J. Theor. Phys. 38, 1113 (1999)] [arXiv:hep-th/9711200].

[2] S. S. Gubser, I. R. Klebanov and A. M. Polyakov, Phys. Lett. B 428, 105 (1998) [arXiv:hep-th/9802109]. E. Witten, Adv. Theor. Math. Phys. 2, 253 (1998) [arXiv:hepth/9802150]. N. Itzhaki, J. M. Maldacena, J. Sonnenschein and S. Yankielowicz, Phys. Rev. D 58, 046004 (1998) [arXiv:hep-th/9802042].

[3] O. Lunin and J. M. Maldacena, JHEP 0505, 033 (2005) [arXiv:hep-th/0502086]. J. Maldacena, D. Martelli and Y. Tachikawa, JHEP 0810, 072 (2008) [arXiv:0807.1100 [hep-th]]. C. P. Herzog, M. Rangamani and S. F. Ross, JHEP 0811, 080 (2008) [arXiv:0807.1099 [hep-th]]. C. P. Herzog, M. Rangamani and S. F. Ross, JHEP 0811, 080 (2008) [arXiv:0807.1099 [hep-th]].

[4] V. Belinski and E. Verdaguer, Cambridge, UK: Univ. Pr. (2001) 258 pA. A. Pomeransky, Phys. Rev. D 73, 044004 (2006) [arXiv:hep-th/0507250]. T. Mishima and H. Iguchi, Phys. Rev. D 73, 044030 (2006) [arXiv:hep-th/0504018].

[5] H. Elvang and P. Figueras, JHEP 0705, 050 (2007) [arXiv:hep-th/0701035].

[6] J. Maldacena and D. Martelli, JHEP 1001, 104 (2010) [arXiv:0906.0591 [hep-th]].

[7] A. Butti, M. Grana, R. Minasian, M. Petrini and A. Zaffaroni, JHEP 0503, 069 (2005) [arXiv:hep-th/0412187]. 
[8] I. R. Klebanov and M. J. Strassler, JHEP 0008, 052 (2000) [arXiv:hep-th/0007191].

[9] R. Casero, C. Nunez and A. Paredes, Phys. Rev. D 73, 086005 (2006) [arXiv:hepth/0602027]. R. Casero, C. Nunez and A. Paredes, Phys. Rev. D 77, 046003 (2008) [arXiv:0709.3421 [hep-th]].

[10] C. Hoyos-Badajoz, C. Nunez and I. Papadimitriou, Phys. Rev. D 78, 086005 (2008) [arXiv:0807.3039 [hep-th]].

[11] A. H. Chamseddine and M. S. Volkov, Phys. Rev. Lett. 79, 3343 (1997) [arXiv:hepth/9707176]. J. M. Maldacena and C. Nunez, Phys. Rev. Lett. 86, 588 (2001) [arXiv:hepth/0008001].

[12] R. Minasian, M. Petrini and A. Zaffaroni, JHEP 1004, 080 (2010) [arXiv:0907.5147 [hepth]].

[13] J. Gaillard, D. Martelli, C. Nunez and I. Papadimitriou, Nucl. Phys. B 843, 1 (2011) [arXiv:1004.4638 [hep-th]].

[14] N. Halmagyi, arXiv:1003.2121 [hep-th].

[15] A. Dymarsky, I. R. Klebanov and N. Seiberg, JHEP 0601, 155 (2006) [arXiv:hepth/0511254].

[16] R. P. Andrews and N. Dorey, Nucl. Phys. B 751, 304 (2006) [arXiv:hep-th/0601098]. R. P. Andrews and N. Dorey, Phys. Lett. B 631, 74 (2005) [arXiv:hep-th/0505107].

[17] E. Witten, Adv. Theor. Math. Phys. 2, 505 (1998) [arXiv:hep-th/9803131].

[18] T. Sakai and S. Sugimoto, Prog. Theor. Phys. 113, 843 (2005) [arXiv:hep-th/0412141].

[19] O. Aharony, J. Sonnenschein and S. Yankielowicz, Annals Phys. 322, 1420 (2007) [arXiv:hep-th/0604161].

[20] J. Gaillard and D. Martelli, arXiv:1008.0640 [hep-th].

[21] C. Nunez, A. Paredes and A. V. Ramallo, Adv. High Energy Phys. 2010, 196714 (2010) [arXiv:1002.1088 [hep-th]].

[22] C. Nunez, I. Papadimitriou and M. Piai, Int. J. Mod. Phys. A 25, 2837 (2010) [arXiv:0812.3655 [hep-th]].

[23] S. S. Gubser, A. A. Tseytlin, and M. S. Volkov, JHEP 0109:017,2001 (JHEP 0109:017,2001).

[24] E. Caceres, R. Flauger, M. Ihl, and T. Wrase, JHEP 0803:020,2008 (Nov., 2007). 
[25] E. Caceres, R. Flauger, and T. Wrase, arXiv:0908.4483.

[26] G. T. Horowitz, A. Strominger, Nucl. Phys. B360, 197-209 (1991).

[27] M. J. Duff, J. X. Lu, Nucl. Phys. B416, 301-334 (1994). [hep-th/9306052].

[28] M. J. Duff, R. R. Khuri, J. X. Lu, Phys. Rept. 259, 213-326 (1995). [hep-th/9412184].

[29] M. Atiyah, J. M. Maldacena and C. Vafa, J. Math. Phys. 42 (2001) 3209 [arXiv:hepth/0011256]. C. Vafa, J. Math. Phys. 42, 2798 (2001) [arXiv:hep-th/0008142].

J. D. Edelstein and C. Nunez, JHEP 0104, 028 (2001) [arXiv:hep-th/0103167].

[30] A. Brandhuber, Nucl. Phys. B 629, 393 (2002) [arXiv:hep-th/0112113].

[31] J. M. Maldacena and H. S. Nastase, JHEP 0109, 024 (2001) [arXiv:hep-th/0105049].

[32] C. G. . Callan, J. A. Harvey and A. Strominger, Nucl. Phys. B 359 (1991) 611.

[33] A. Buchel, Nucl. Phys. B 600 (2001) 219 [arXiv:hep-th/0011146]. A. Buchel, C. P. Herzog, I. R. Klebanov, L. A. Pando Zayas and A. A. Tseytlin, JHEP 0104 (2001) 033 [arXiv:hepth/0102105]. S. S. Gubser, C. P. Herzog, I. R. Klebanov and A. A. Tseytlin, JHEP 0105 (2001) 028 [arXiv:hep-th/0102172]. L. A. Pando Zayas and C. A. Terrero-Escalante, JHEP 0609 (2006) 051 [arXiv:hep-th/0605170]. O. Aharony, A. Buchel and P. Kerner, Phys. Rev. D 76 (2007) 086005 [arXiv:0706.1768 [hep-th]]. M. Mahato, L. A. Pando Zayas and C. A. Terrero-Escalante, JHEP 0709 (2007) 083 [arXiv:0707.2737 [hep-th]]. A. Buchel, arXiv:1012.2404 [hep-th].

[34] G. T. Horowitz and D. L. Welch, Phys. Rev. D 49, 590 (1994) [arXiv:hep-th/9308077].

[35] M. Schvellinger, JHEP 0409, 057 (2004) [arXiv:hep-th/0407152]. P. McGuirk, G. Shiu and Y. Sumitomo, Nucl. Phys. B 842, 383 (2010) [arXiv:0910.4581 [hep-th]].

[36] I. Bena, M. Grana and N. Halmagyi, JHEP 1009, 087 (2010) [arXiv:0912.3519 [hep-th]]. I. Bena, G. Giecold and N. Halmagyi, arXiv:1011.2195 [hep-th].

[37] J. Gaillard and J. Schmude, JHEP 1002, 032 (2010) [arXiv:0908.0305 [hep-th]].

[38] F. Bigazzi, A. L. Cotrone, J. Mas, A. Paredes, A. V. Ramallo and J. Tarrio, JHEP 0911, 117 (2009) [arXiv:0909.2865 [hep-th]]. F. Bigazzi, A. L. Cotrone and J. Tarrio, JHEP 1002, 083 (2010) [arXiv:0912.3256 [hep-th]]. F. Bigazzi and A. L. Cotrone, JHEP 1008, 128 (2010) [arXiv:1006.4634 [hep-ph]]. M. Mia, K. Dasgupta, C. Gale and S. Jeon, Nucl. Phys. B 839 (2010) 187 [arXiv:0902.1540 [hep-th]]. F. Bigazzi, A. L. Cotrone, J. Mas, D. Mayerson and J. Tarrio, arXiv:1101.3560 [hep-th].

[39] F. Chen, K. Dasgupta, P. Franche, S. Katz and R. Tatar, arXiv:1007.5316 [hep-th]. 